\title{
Rescaling Ward Identities in the Random Normal Matrix Model
}

\author{
Yacin Ameur ${ }^{1}$ • Nam-Gyu Kang² . \\ Nikolai Makarov ${ }^{3}$
}

Received: 18 September 2017 / Revised: 12 February 2018 / Accepted: 26 February 2018 /

Published online: 2 April 2018

(C) The Author(s) 2018

\begin{abstract}
We study spacing distribution for the eigenvalues of a random normal matrix, in particular at points on the boundary of the spectrum. Our approach uses Ward's (or the "rescaled loop") equation — an identity satisfied by all sequential limits of the rescaled one-point functions.
\end{abstract}

Keywords Random normal matrix · Universality · Ward's equation · Translation invariance

Mathematics Subject Classification Primary 60B20; Secondary 60G55 - 81T40 • 30C40 - 30D15 - 35R09

Communicated by Arno Kuijlaars.

Nam-Gyu Kang was supported by Samsung Science and Technology Foundation (SSTF-BA1401-01). Nikolai Makarov was supported by NSF Grant No. 1500821.

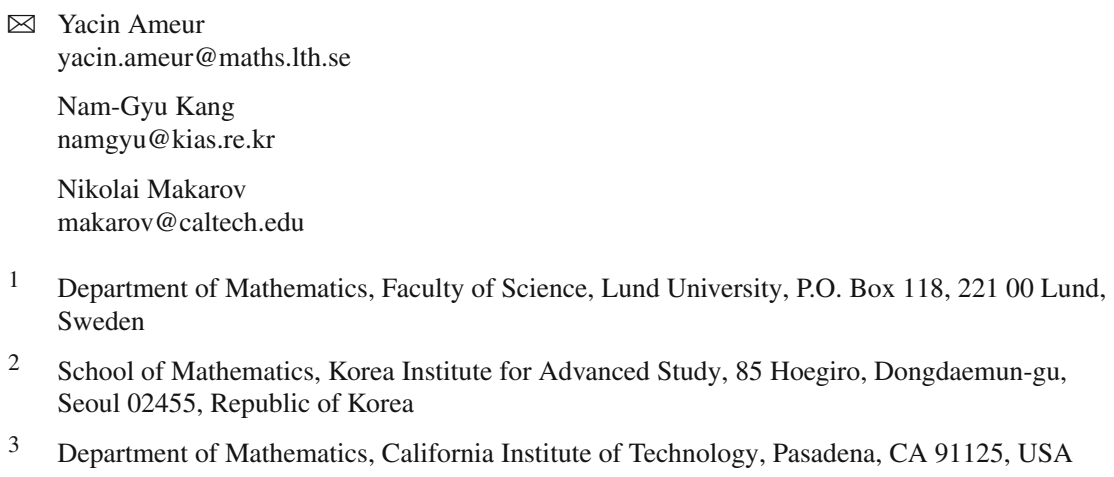


Consider a normal matrix $M$ (i.e., $M M^{*}=M^{*} M$ ) of some large order $n$, picked randomly with respect to a probability measure of the form

$$
d \mu_{n}(M)=\frac{1}{\mathcal{Z}_{n}} e^{-n \operatorname{tr} Q(M)} d M .
$$

Here $d M$ is the surface measure on normal $n \times n$ matrices inherited from $2 n^{2}$ dimensional Lebesgue measure via the natural embedding into $\mathbb{C}^{n^{2}}, Q(\zeta)$ is a suitable real-valued function defined on $\mathbb{C}$ ("large" as $\zeta \rightarrow \infty$ ), and $\mathcal{Z}_{n}$ is a normalizing constant; $\operatorname{tr} Q(M)=\sum_{1}^{n} Q\left(\zeta_{j}\right)$ is the usual trace of the matrix $Q(M)$, where $\zeta_{j}$ denote the eigenvalues.

A random sample $\left\{\zeta_{j}\right\}_{1}^{n}$, where $\zeta_{j}$ are the eigenvalues of $M$, is known as a random normal eigenvalue ensemble. It is convenient to briefly call it a "system."

If $Q$ is just defined on $\mathbb{R}$ and $d M$ is surface measure on the Hermitian matrices (i.e., $M^{*}=M$ ), we have random Hermitian matrices. The study of such ensembles, e.g., using the technique of Riemann-Hilbert problems, is a classical and active area of research.

As $n \rightarrow \infty$, the system tends to occupy a certain compact set $S$ called the droplet. We will here fix a point $p \in S$ and study microscopic properties of the system near $p$. This corresponds to the study of spacing distribution in Hermitian theory.

The simplest case is the well-known Ginibre ensemble, in which $Q(\zeta)=|\zeta|^{2}$ and $S$ is the closed unit disc. In this case, the system $\left\{\zeta_{j}\right\}_{1}^{n}$ can be interpreted as the eigenvalues of an $n \times n$ matrix whose entries are i.i.d. complex, centered Gaussian random variables of variance $1 / n$. We note a few facts about this ensemble.

If we rescale about the point $p=0$ in the interior of $S$, by letting $z_{j}=\sqrt{n} \zeta_{j}$, and let $n \rightarrow \infty$, then simple calculation shows that the rescaled system $\left\{z_{j}\right\}_{1}^{n}$ converges to a determinantal random point field with the correlation kernel

$$
G(z, w):=e^{z \bar{w}-|z|^{2} / 2-|w|^{2} / 2} .
$$

It is convenient to refer to $G$ as the Ginibre kernel and the corresponding point field as the "bulk Ginibre point field."

If we instead rescale about the boundary point $p=1$ by $z_{j}=\sqrt{n}\left(\zeta_{j}-1\right)$, then again there is a limiting point field, but the correlation kernel turns out to be

$$
K(z, w)=G(z, w) F(z+\bar{w})
$$

where $F$ is the entire function given by

$$
F(z)=\frac{1}{2} \operatorname{erfc}\left(\frac{z}{\sqrt{2}}\right) \equiv \frac{1}{\sqrt{2 \pi}} \int_{z}^{\infty} e^{-\zeta^{2} / 2} d \zeta
$$

Here "erfc" is the complementary error function, while $F$ is closely related to the "plasma dispersion function" in the physics literature, see [19]. Sometimes we will refer to $F$ as the plasma function (Figs. 1, 2). 


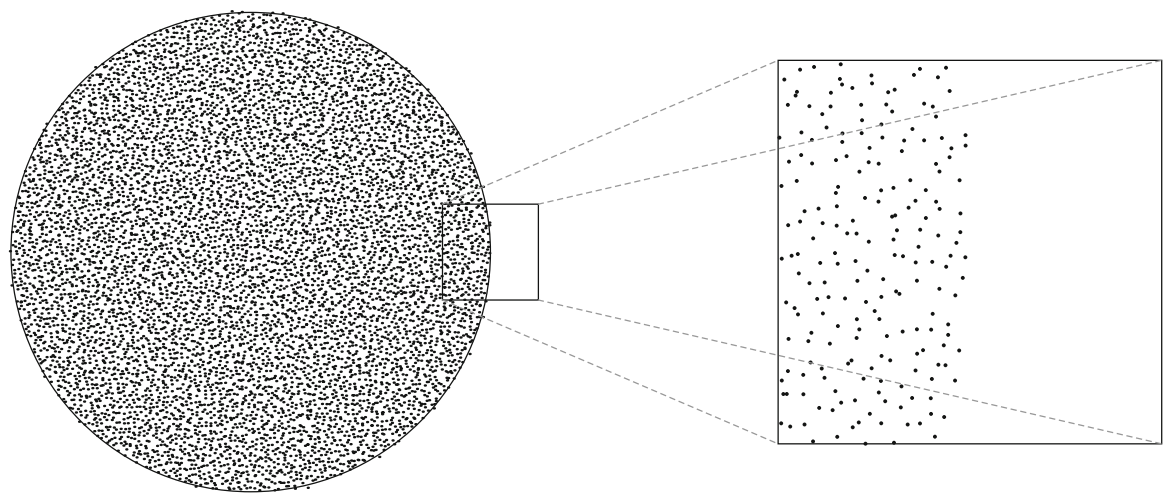

Fig. 1 A sample of the free boundary Ginibre process for a large value of $n$
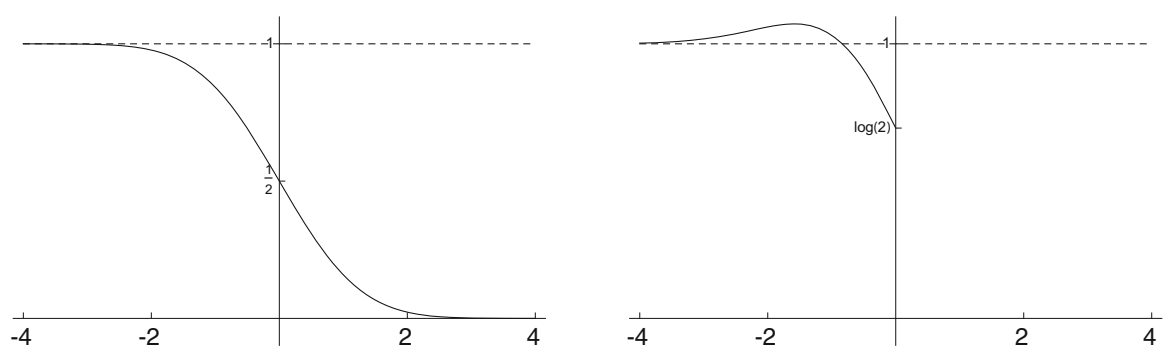

Fig. 2 The free boundary and hard edge profiles, respectively

To the best of our knowledge, the above formula first appeared in the paper [18] by Forrester and Honner; cf. [10,12] for some alternative proofs. We call the point field with the correlation kernel $(0.2)$ the "boundary Ginibre point field."

We will start this paper with an alternative simple argument that depends on the normal approximation of the Poisson distribution. This argument works in some other situations as well. In particular, we show how to use it to compute the limiting kernel of the hard edge Ginibre ensemble, where the potential is redefined as $+\infty$ outside the unit disc $S$. In this case, we get

$$
K(z, w)=G(z, w) H(z+\bar{w}) \mathbf{1}_{\mathbb{L}}(z) \mathbf{1}_{\mathbb{L}}(w),
$$

where $\mathbf{1}_{\mathbb{L}}$ is the indicator function for the left half-plane $\mathbb{L}=\{z ; \operatorname{Re} z<0\}$,

$$
H(z)=\frac{1}{\sqrt{2 \pi}} \int_{-\infty}^{0} \frac{e^{-(z-t)^{2} / 2}}{F(t)} d t
$$

and $F$ is given by (0.3) (cf. Fig. 2).

Analogously to well-known "universality" results in the theory of random Hermitian matrices, where the well-known sine, Airy, and Bessel processes crop up, one can hope to find analogous processes in the normal matrix case. Here the starting point is the well-known fact (observed in [2]) that if we rescale properly at a point $p$ in the interior 


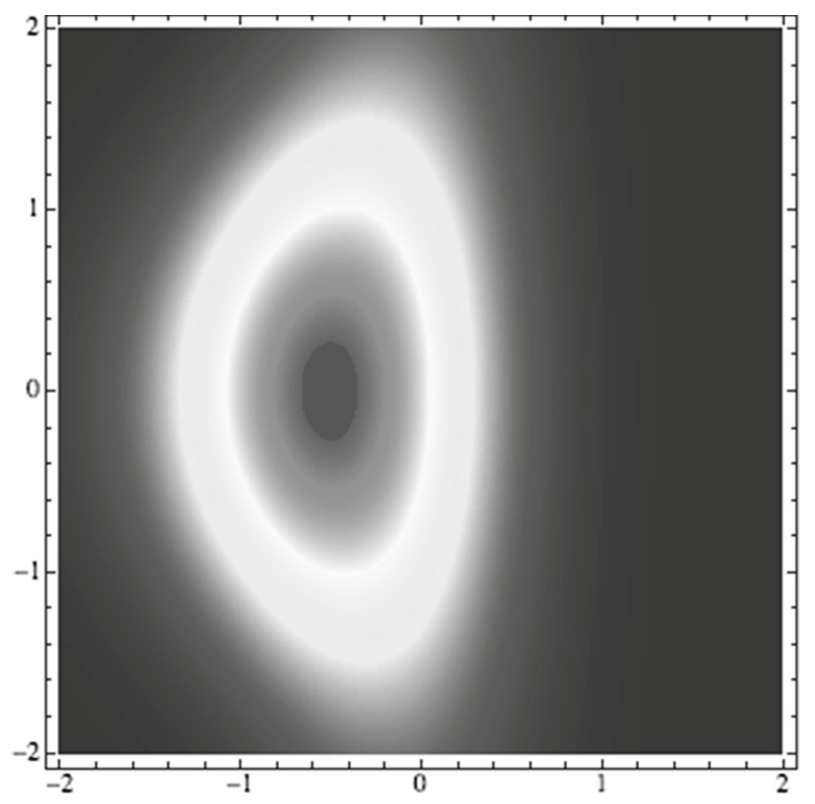

Fig. 3 The level sets of the Berezin kernel $B(0, w)$ for the free boundary Ginibre ensemble

of $S$ with $\Delta Q(p)>0$, then the rescaled processes converge to the bulk Ginibre point field.

Full universality at boundary points remains an open problem, but we will here obtain some partial results by suggesting and exploring a new approach that is based on rescaling of Ward's identities (or loop equations). This approach is of interest also in other contexts, see, e.g., [1,8].

We will derive a "universal" (independent of $Q$ ) equation that is satisfied by all sequential limits $K$ of the rescaled correlation kernels. In the case of a regular point on the free boundary, the equation has the following form:

$$
\bar{\partial} C=R-1-\partial \bar{\partial} \log R,
$$

where $R(z)=K(z, z)$ is the intensity 1 -point function and

$$
C(z)=\frac{1}{\pi} \int_{\mathbb{C}} \frac{B(z, w)}{z-w} d^{2} w
$$

is the Cauchy transform of the Berezin kernel $B(z, w)$ (see Fig. 3),

$$
B(z, w)=\frac{|K(z, w)|^{2}}{K(z, z)} .
$$

Equation (0.6) is an equation for $R=R(z)$ because (as we'll see) $R$ determines $K$ and therefore $B$ and $C$ by means of analytic continuation. 
The analysis of equation (0.6) is one of the main points of this paper. We will describe all (vertically) translation invariant solutions $R$ to Ward's equation, i.e., solutions for which $R(z)$ depends only on $\operatorname{Re} z$. If we rescale properly about a regular boundary point, the boundary of the droplet will approach the imaginary axis, making it very plausible that the intensity of eigenvalues should be translation invariant. We will obtain results that strongly indicate that this is in fact the case, but we are not able to completely settle the issue here. It is however easy to verify translation invariance for radially symmetric potentials, so we do get universality for that class.

The method of rescaled Ward identities is quite general and can be used in a variety of different settings. In addition to the already mentioned cases of regular points in the bulk, or on a free or hard edge boundary, one can consider various types of singular points as well. We get certain equations that depend only on the setting but not on $Q$ ("universality"). We will derive such Ward's equations in several settings but will focus most on the regular free boundary case (0.2). The analysis of other cases appear elsewhere, see [5,6,8].

A detailed description of our results is given in the following section.

Notational conventions. By $D(\zeta ; r)$ we denote the open disc with center $\zeta$ and radius $r$. We write $\partial E$, Int $E, \mathrm{cl} E$, and $E^{c}$ for the boundary, the interior, the closure, and the complement of a set $E \subset \mathbb{C}$. The indicator function of a set $E$ is denoted by $\mathbf{1}_{E}$. We write $\partial=\frac{1}{2}(\partial / \partial x-i \partial / \partial y)$ and $\bar{\partial}=\frac{1}{2}(\partial / \partial x+i \partial / \partial y)$ for the complex derivatives and $\Delta=\partial \bar{\partial}$ for the normalized Laplacian. Thus $\Delta$ is $\frac{1}{4}$ times the standard Laplacian. We write $d A(z)=d^{2} z / \pi$ for normalized Lebesgue measure. Thus the unit disc has measure 1 . The volume measure on $\mathbb{C}^{k}$ is defined by $d V_{k}\left(\zeta_{1}, \ldots, \zeta_{k}\right)=$ $d A\left(\zeta_{1}\right) \cdots d A\left(\zeta_{k}\right)$. The intensities and the correlation kernel of the original point processes are boldfaced as $\mathbf{R}_{n, k}$ and $\mathbf{K}_{n}$, respectively, and those of the rescaled point processes are italicized as $R_{n, k}$ and $K_{n}$, respectively.

A continuous function $f: \mathbb{C}^{2} \rightarrow \mathbb{C}$ is termed Hermitian if $f(z, w)=\overline{f(w, z)}$. We shall say that $f$ is Hermitian-analytic (or Hermitian-entire) if $f$ is Hermitian and analytic (entire, respectively) as a function of $z$ and $\bar{w}$. A Hermitian-entire function is uniquely determined by its values $f(z, z)$ on the diagonal, see [23, Lemma 2.5.1]. A Hermitian function $c$ is called a cocycle if $c(z, w)=g(z) \overline{g(w)}$ for a continuous unimodular function $g$. The determinant of a matrix $\left(m_{i j}\right)$ remains unchanged if we multiply each $m_{i j}$ by $c\left(z_{i}, z_{j}\right)$.

\section{Introduction and Results}

\subsection{Potential Theory and Droplets}

Fix a suitable function ("external potential") $Q: \mathbb{C} \rightarrow \mathbb{R} \cup\{+\infty\}$. Let $\mathcal{P}$ denote the class of positive, compactly supported Borel measures on $\mathbb{C}$.

Define the weighted logarithmic energy of $\mu \in \mathcal{P}$ in external field $Q$ by

$$
I_{Q}[\mu]=\iint_{\mathbb{C}^{2}} \log \frac{1}{|\zeta-\eta|} d \mu(\zeta) d \mu(\eta)+\int_{\mathbb{C}} Q d \mu .
$$


Thinking of $\mu$ as the distribution of an electric charge, $I_{Q}[\mu]$ is the sum of the Coulomb interaction energy and the energy of interaction of $\mu$ with the external field $Q$.

We always assume that $Q$ is lower semi-continuous and that $Q$ is finite on some set of positive logarithmic capacity. We will also assume that $Q$ is sufficiently large at infinity so as to confine the system to a finite portion of the plane. To be precise, it suffices to assume that $\lim \inf _{\zeta \rightarrow \infty} Q(\zeta) / \log |\zeta|>2$.

A classical theorem of Frostman states that there exists a unique equilibrium measure $\sigma$ that minimizes the weighted energy,

$$
I_{Q}[\sigma]=\min _{\mu(\mathbb{C})=1} I_{Q}[\mu], \quad(\mu \in \mathcal{P})
$$

See [32]. We denote the compact support of the equilibrium measure by

$$
S=S[Q]=\operatorname{supp} \sigma
$$

We refer to $S$ as the droplet in the external field $Q$. It is known that if $Q$ is smooth in some neighborhood of $S$, then $\sigma$ is absolutely continuous and takes the explicit form (see [32])

$$
d \sigma=\mathbf{1}_{S} \cdot \Delta Q d A
$$

Since $\sigma$ is a probability measure, we have $\Delta Q \geq 0$ on $S$.

Our main assumptions throughout are that there is some neighborhood $\Omega$ of $S$ such that

$Q$ is real analytic in $\Omega$,

$$
\Delta Q>0 \text { in } \Omega \text {. }
$$

With these assumptions, the complement $S^{c}$ has a local Schwarz function at each boundary point, and we can rely on the fundamental theorem of Sakai [33] concerning domains with local Schwarz functions. In particular, we can apply Sakai's regularity theorem, which implies that all but finitely many boundary points $p \in \partial S$ are regular in the sense that there is a disc $D=D(p ; \epsilon)$ such that $D \backslash S$ is a Jordan domain and $D \cap(\partial S)$ is a real analytic arc. A nonregular point $p \in \partial S$ is called a singular boundary point. Such points can be classified further as cusps or double points.

\subsection{Rescaling Eigenvalue Ensembles}

Consider a random eigenvalue ensemble $\left\{\zeta_{j}\right\}_{1}^{n}$ from (0.1). It is well known that we can alternatively regard the system as being picked randomly with respect to the Boltzmann-Gibbs law,

$$
d \mathbb{P}_{n}(\zeta)=\frac{1}{Z_{n}} e^{-H_{n}(\zeta)} d V_{n}(\zeta), \quad \zeta=\left(\zeta_{1}, \ldots, \zeta_{n}\right) \in \mathbb{C}^{n}
$$


where $H_{n}$, the Hamiltonian, is defined by

$$
H_{n}\left(\zeta_{1}, \ldots, \zeta_{n}\right)=\sum_{j \neq k} \log \frac{1}{\left|\zeta_{j}-\zeta_{k}\right|}+n \sum_{j=1}^{n} Q\left(\zeta_{j}\right)
$$

The normalizing constant $Z_{n}=\int e^{-H_{n}} d V_{n}$ is the so-called partition function.

We can thus think of the point-process $\left(\zeta_{j}\right)_{1}^{n}$ either as a system of repelling pointcharges in external field $n Q$, with logarithmic interactions, or as eigenvalues of random normal matrices from the ensemble above.

An important feature of this kind of point process is that it is determinantal. This means that if $\mathbf{R}_{n, k}$ denotes the $k$-point intensity function of the process $\left\{\zeta_{j}\right\}_{1}^{n}$, then

$$
\mathbf{R}_{n, k}\left(\zeta_{1}, \ldots, \zeta_{k}\right)=\operatorname{det}\left(\mathbf{K}_{n}\left(\zeta_{i}, \zeta_{j}\right)\right)_{i, j=1}^{k}
$$

where $\mathbf{K}_{n, k}$ is a certain Hermitian function known as a correlation kernel. Indeed, by Dyson's determinant formula, given in, e.g., [32, Section IV.7.2] or [17,28], we have the formula

$$
\mathbf{K}_{n}(\zeta, \eta)=\sum_{j=0}^{n-1} q_{j}(\zeta) \bar{q}_{j}(\eta) e^{-n Q(\zeta) / 2-n Q(\eta) / 2}
$$

Here $q_{j}$ is the $j$ :th orthonormal polynomial with respect to the measure $e^{-n Q(\zeta)} d A(\zeta)$.

We remind the reader that the $k$-point function of $\mathbb{P}_{n}$ is defined for distinct points $\eta_{j}$ by

$$
\mathbf{R}_{n, k}\left(\eta_{1}, \ldots, \eta_{k}\right)=\lim _{\varepsilon \rightarrow 0}\left[\varepsilon^{-2 k} \cdot \mathbb{E}_{n}\left(\prod_{j=1}^{k} N_{D\left(\eta_{j} ; \varepsilon\right)}\right)\right]
$$

Here $N_{B}$ is the number of points $\zeta_{j}$ that fall in a set $B ; \mathbb{E}_{n}$ is expectation with respect to $\mathbb{P}_{n}$.

It should be mentioned that the so-called $\beta$-ensembles corresponding to the Boltzmann-Gibbs law $d \mathbb{P}_{n}^{(\beta)}(\zeta) \propto e^{-\beta H_{n}(\zeta)} d V_{n}(\zeta)$ are not determinantal if $\beta \neq 1$, and some aspects of the methods we develop in this manuscript do not work for them.

We are interested in microscopic properties of the system $\left\{\zeta_{j}\right\}_{1}^{n}$ near a point $p \in S$. It is natural to magnify distances about $p$ by a factor $\sqrt{n \Delta Q(p)}$. We also fix an angle $\theta \in \mathbb{R}$; when $p$ is a regular boundary point, we choose $\theta$ so that $e^{i \theta}$ as the outer normal to $\partial S$ at $p$; in other cases, we may choose it arbitrarily.

Consider the rescaled system $\Theta_{n}=\left\{z_{j}\right\}_{1}^{n}$, given by

$$
z_{j}=e^{-i \theta} \sqrt{n \Delta Q(p)} \cdot\left(\zeta_{j}-p\right), \quad j=1, \ldots, n
$$

We can choose the coordinate system so that $p=0$ and $\theta=0$. This will be done hereafter, except in some cases where other choices are natural. More generally, we can rescale about an $n$-dependent point $p=p_{n}$, or alternatively, let 0 denote the origin 
in an $n$-dependent coordinate system. This generalization presents no new difficulties as long as the sequence $p_{n}$ is contained in a sufficiently small neighborhood of the droplet and satisfies the decisive condition $\Delta Q\left(p_{n}\right) \geq$ const. $>0$. See $[1,6]$.

The law of $\Theta_{n}$ is defined as the image of the Boltzmann-Gibbs measure (1.1) under the map (1.4). The rescaled system $\Theta_{n}$ then has intensities denoted by $R_{n, k}$, where

$$
R_{n, k}\left(z_{1}, \ldots, z_{k}\right)=\frac{1}{(n \Delta Q(0))^{k}} \mathbf{R}_{n, k}\left(\zeta_{1}, \ldots, \zeta_{k}\right)
$$

The point process $\Theta_{n}$ is determinantal with kernel $K_{n}$ given by

$$
K_{n}(z, w)=\frac{1}{n \Delta Q(0)} \mathbf{K}_{n}(\zeta, \eta), \quad \text { where }\left\{\begin{array}{l}
z=\sqrt{n \Delta Q(0)} \cdot \zeta \\
w=\sqrt{n \Delta Q(0)} \cdot \eta
\end{array}\right.
$$

The fundamental problem is existence and uniqueness of a limiting point field of the rescaled processes $\Theta_{n}$ as $n \rightarrow \infty$. For our purposes, convergence will mean locally uniform convergence of all intensities $R_{n, k}$ to some limits $R_{k}$ as $n \rightarrow \infty$. Whenever this is the case, $R_{k}$ can be interpreted as a $k$-point function for a "point field" in $\mathbb{C}$, meaning a probability law on a suitable space of infinite configurations $\left\{z_{i}\right\}_{1}^{\infty} \subset \mathbb{C}$ (cf. [34], see also a forthcoming version of [6]).

It suffices here to note that the desired convergence of the processes $\Theta_{n}$ holds if the correlation kernels $K_{n}$ converge to a limit $K$ locally uniformly on $\mathbb{C}^{2}$. Moreover, the limiting point field is uniquely determined by $K$ if the functions $K_{n}(z, z)$ are uniformly bounded, and it is then determinantal with intensity functions

$$
R_{k}\left(z_{1}, \ldots, z_{k}\right)=\operatorname{det}\left(K\left(z_{i}, z_{j}\right)\right)_{i, j=1}^{k}
$$

More generally, if $K_{n}$ is a correlation kernel and $\left(c_{n}\right)_{1}^{\infty}$ is a sequence of cocycles, then $c_{n} K_{n}$ is another kernel giving rise to the same joint intensities $R_{n, k}$. The problem is thus to show that there exists a sequence of cocycles such that $c_{n} K_{n}$ converges locally uniformly to a nontrivial limit $K$ with bounded convergence on the diagonal in $\mathbb{C}^{2}$.

\subsection{Compactness, Nontriviality, and Ward's Equation}

Our first theorem states the existence of sequential limits of the rescaled point processes $\Theta_{n}$ and specifies the form of limiting correlation kernels.

Theorem 1.1 (i) There is a sequence $c_{n}$ of cocycles such that every subsequence of $\left(c_{n} K_{n}\right)_{1}^{\infty}$ has a further subsequence that converges uniformly to some limit $K$ on compact subsets of $\mathbb{C}^{2}$.

(ii) Each $K$ in (i) has the form $K=G \Psi$, where $\Psi$ is a Hermitian entire function and $G$ is the Ginibre kernel. 
Analyticity of $\Psi(z, \bar{w})$ allows us to use analytic continuation. It is clear that a limiting kernel $K$ in Theorem 1.1 is a positive kernel in Aronszajn's sense [9]; i.e., for all finite sequences $\left(z_{j}\right)_{1}^{N}$ of points and all choices of scalars $\left(\alpha_{j}\right)_{1}^{N}$, we have

$$
\sum_{j, k=1}^{N} \alpha_{j} \bar{\alpha}_{k} K\left(z_{j}, z_{k}\right) \geq 0 .
$$

The next theorem describes some additional positivity properties of a limiting kernel. We denote by $L_{a}^{2}(\mu)$ the Bargmann-Fock space of entire functions square-integrable with respect to the measure

$$
d \mu(z)=e^{-|z|^{2}} d A(z) .
$$

Theorem 1.2 Let $K=G \Psi$ be any limiting kernel in Theorem 1.1.

(i) K satisfies the following "mass-one inequality":

$$
\text { for all } z \in \mathbb{C}, \quad \int_{\mathbb{C}}|K(z, w)|^{2} d A(w) \leq K(z, z) .
$$

(ii) Let $L(z, w):=e^{z \bar{w}} \Psi(z, w)$. Then $L$ is the reproducing kernel for a certain Hilbert space $\mathcal{H}_{*}$ of entire functions that sits contractively in $L_{a}^{2}(\mu)$.

(iii) Also we have

$$
0 \leq K \leq G
$$

in the sense that $K$ and $G-K$ are positive kernels.

By the general theory mentioned in the previous subsection, a limiting kernel $K$ is the correlation kernel of some point field in the plane, which we call a limiting point field. The 1-point function of this point field is denoted by $R(z)=K(z, z)$. If $R$ does not vanish on $\mathbb{C}$, we can consider the Berezin kernel $B(z, w)=|K(z, w)|^{2} / K(z, z)$ and define the Cauchy transform

$$
C(z)=\int_{\mathbb{C}} \frac{B(z, w)}{z-w} d A(w) .
$$

Theorem 1.3 Let $K=G \Psi$ be a limiting kernel in Theorem 1.1.

(i) Either $R$ is trivial, in the sense that $R=0$ identically in $\mathbb{C}$, or $R>0$ everywhere.

(ii) Ward's equation: if $R$ is nontrivial, then the integral $C(z)$ in (1.7) converges and defines a smooth function such that

$$
\bar{\partial} C(z)=R(z)-1-\Delta \log R(z), \quad z \in \mathbb{C} .
$$


The first part of this theorem says that we have a dichotomy (some sort of "zero-one law"). Both cases are possible. The next theorem implies that we have nontriviality at regular boundary points, whereas triviality (i.e. $R \equiv 0$ ) occurs, for example, if $p$ is a singular boundary point, see [6].

\subsection{A Priori Estimates}

Ward's equation has many solutions. In order to fix a solution uniquely, we need to know that certain additional conditions are satisfied. These conditions depend on the nature of the point we are rescaling about. For regular points, the results are as follows.

Theorem 1.4 Suppose that 0 is a regular boundary point, and let $K$ be a corresponding limiting kernel in Theorem 1.1. Write $x=\operatorname{Re} z$.

(i) Exterior estimate: There is a constant $C$ such that

$$
R(z) \leq C e^{-2 x^{2}} \text { for } x \geq 0 \text {. }
$$

(ii) Interior estimate: If $\ell$ is any number with $\ell<1 / 2$, then there is a constant $C$ (depending on $\ell$ ) such that

$$
0 \leq 1-R(z) \leq C e^{-\ell x^{2}} \text { for } x \leq 0
$$

Theorem 1.5 Assume that the droplet $S$ is connected and all boundary points of $S$ are regular. For almost all boundary points, the following identity holds for all limiting kernels:

$$
\int_{-\infty}^{+\infty} x \cdot\left(R(x)-\mathbf{1}_{(-\infty, 0)}(x)\right) d x=\frac{1}{8} .
$$

\subsection{Translation Invariant Solutions to Ward's Equation}

Let $K=G \Psi$ be a limiting kernel in Theorem 1.1. We say that $\Psi$ (or $K$ ) is (vertically) translation invariant (in short: $t . i$.) if

$$
\Psi(z+i t, w+i t)=\Psi(z, w), \quad t \in \mathbb{R} .
$$

In this case, $\Psi$ can be represented in the form

$$
\Psi(z, w)=\Phi(z+\bar{w})
$$

for some entire function $\Phi$. We can describe a relevant class of such $\Phi$ by means of the "Gaussian kernel"

$$
\gamma(z):=\frac{1}{\sqrt{2 \pi}} e^{-z^{2} / 2}, \quad z \in \mathbb{C} .
$$


Let $f$ be a bounded function on $\mathbb{R}$. Then we define the entire function $\gamma * f$ by the equation

$$
(\gamma * f)(z)=\int_{-\infty}^{+\infty} \gamma(z-t) f(t) d t, \quad z \in \mathbb{C},
$$

so $\gamma * f$ is the analytic continuation of usual convolution in $\mathbb{R}$. For example, the plasma function $F$ in $(0.3)$ can be represented as

$$
F(z)=\gamma * \mathbf{1}_{(-\infty, 0)}(z)=\int_{-\infty}^{0} \gamma(z-t) d t .
$$

We will see that positivity properties of the limiting kernels in Theorem 1.2 imply that if the kernel is t.i., the entire function $\Phi$ in (1.8) in has the form $\Phi=\gamma * f$ with a bounded function $f$.

Theorem 1.6 Let $\Phi=\gamma * f$, where $f$ is a bounded function. Then the kernel $K(z, w)=G(z, w) \Phi(z+\bar{w})$ satisfies Ward's equation if and only if $\Phi=\gamma * \mathbf{1}_{I}$ for some connected set $I \subset \mathbb{R}$.

Using a priori estimates in Theorem 1.4, we obtain the following corollary.

Theorem 1.7 If the limiting kernel $K$ at a regular boundary point is translation invariant, then $K(z, w)=G(z, w) \Phi(z+\bar{w})$ and there is some a such that

$$
\Phi=\gamma * \mathbf{1}_{(-\infty, a)}
$$

Since $a=0$ is the only choice of $a$ consistent with the $1 / 8$-formula in Theorem 1.5 , we have the following result for radially symmetric potentials, $Q(z)=Q(|z|)$.

Theorem 1.8 Assume that the droplet $S$ is connected. If $Q$ is radially symmetric and $p$ is any boundary point, then the rescaled point processes converge to the boundary Ginibre point field.

As we mentioned, it is natural to expect that the boundary Ginibre point process is universal at all regular boundary points for all potentials. A possible approach to ruling out the existence of non-translation-invariant solutions, which satisfy the given apriori estimates, is outlined in Section 8.3.

Recently (after this work was completed) Lee and Riser [25] established this type of convergence for the nonsymmetric "elliptic" potentials $Q=|\zeta|^{2}-t \operatorname{Re}\left(\zeta^{2}\right), 0<$ $t<1$. Their proof depends on explicit computations with the orthogonal polynomials in [31]. Very recently, it seems that universality has been verified in a rather satisfactory generality, by Hedenmalm and Wennman [21]. The approach there depends on a new asymptotic formula for planar orthogonal polynomials. Our approach is quite different, and in fact both methods seem to be of independent interest.

Remark The solutions $\Phi=\gamma * \mathbf{1}_{I}$ with bounded intervals $I$ in Theorem 1.6 seem to be of interest in the connection with the study of singular boundary points, see [6]. 


\subsection{Berezin Kernel and Mass-One Equation}

Let $B_{n}$ be the Berezin kernels of the rescaled processes $\Theta_{n}$, i.e.,

$$
B_{n}(z, w)=\frac{\left|K_{n}(z, w)\right|^{2}}{K_{n}(z, z)} \text {. }
$$

We have for all $z$,

$$
\int_{\mathbb{C}} B_{n}(z, w) d A(w)=1 .
$$

This equation is clear, e.g., from the probabilistic interpretation of $B_{n}$ :

$$
B_{n}(z, w)=R_{n}(w)-R_{n-1}^{(z)}(w),
$$

where $R_{n}=R_{n, 1}$ is the 1-point function for the $n$-point process and $R_{n-1}^{(z)}$ is the 1 -point function for the conditional $(n-1)$-point process $\Theta_{n} \mid\left\{z \in \Theta_{n}\right\}$.

If $K$ is a limiting kernel, we define

$$
B(z, w)=\frac{|K(z, w)|^{2}}{K(z, z)}
$$

We say that $K$ satisfies the mass-one equation if for all $z$,

$$
\int_{\mathbb{C}} B(z, w) d A(w)=1
$$

We will see that the mass-one equation is technically quite similar to Ward's equation and has a nice spectral interpretation: the Hilbert space $\mathcal{H}_{*}$ of entire functions in Theorem 1.2 (ii) sits isometrically in the Fock space.

We can describe solutions to the mass-one equation in the $t . i$. case.

Theorem 1.9 Let $\Phi=\gamma * f$, where $f$ is a bounded function. Then the kernel $K(z, w)=G(z, w) \Phi(z+\bar{w})$ satisfies the mass-one equation if and only if there is a Borel set $E \subset \mathbb{R}$ of positive measure such that $f=\mathbf{1}_{E}$.

\subsection{Organization of the Paper}

In Section 2, we consider the boundary Ginibre ensembles, both for the free boundary and the hard edge. We give a short proof of the convergence of rescaled ensembles to the boundary Ginibre point fields with kernels (0.2) and (0.4), respectively.

In Section 3, we prove Theorem 1.1 (compactness and analyticity) and Theorem 1.2 (mass-one inequality and positivity of $K$ and $G-K$ ).

In Section 4, we derive Ward's equation and prove Theorem 1.3.

In Section 5, we establish a priori bounds for regular points (Theorem 1.4). We also prove the $1 / 8$-formula in Theorem 1.5 at almost every boundary point. 
In Section 6, we specialize to $t$.i. solutions and prove Theorems 1.6-1.9.

In Section 7, we write down versions of Ward's equation in some settings (hard edge, bulk singularities, and $\beta$-ensembles) that are different from the free boundary case discussed above.

The last section, Section 8, contains some general remarks. We relate our methods and results to classical asymptotics for sections of power series. We discuss Hilbert spaces of entire functions associated with limiting kernels. We also comment on the nature of the mass-one equation and Ward's equation and show that they take the form of twisted convolution equations.

\section{The Ginibre Ensembles}

\subsection{Principles of Notation}

Consider first a general potential $Q$. By a weighted polynomial of order $n$, we mean a function of the form $f=q \cdot e^{-n Q / 2}$, where $q$ is an (analytic) polynomial of degree at most $n-1$. Let $\mathcal{W}_{n}$ denote the space of all weighted polynomials of order $n$, considered as a subspace of $L^{2}=L^{2}(\mathbb{C}, d A)$. It is well known that the reproducing kernel $\mathbf{K}_{n}(\zeta, \eta)$ for the space $\mathcal{W}_{n}$ is a correlation kernel for the process $\left\{\zeta_{j}\right\}_{1}^{n}$ corresponding to $Q$. This implies that one has the formula $(1.3)$ for $\mathbf{K}_{n}(\zeta, \eta)$. Recall the Ginibre potential $Q=|\zeta|^{2}$. The corresponding droplet is $S=\{\zeta ;|\zeta| \leq 1\}$.

We shall give an elementary proof for convergence to the boundary Ginibre point field using Poisson approximation of the normal distribution. Our proof is somewhat similar to the argument in the paper [30], where the spectral radius of a Ginibre matrix is studied.

\subsection{Free Boundary Ginibre Ensemble}

Let $\left\{\zeta_{j}\right\}_{1}^{n}$ denote a random configuration for the free boundary Ginibre process. We rescale about the boundary point $p=1$ in the outer normal direction, via $z_{j}=$ $\sqrt{n}\left(\zeta_{j}-1\right)$, writing $\Theta_{n}=\left\{z_{j}\right\}_{1}^{n}$ for the rescaled process. We shall prove the following theorem, found in [18] (cf. [12]).

Theorem 2.1 The processes $\Theta_{n}$ converge to the boundary Ginibre point field as $n \rightarrow$ $\infty$ with locally uniform convergence of intensity functions.

Since $K(z, z)<1$, it suffices to prove the statement about convergence of intensity functions.

By (1.3), a correlation kernel for the process $\left\{\zeta_{j}\right\}_{1}^{n}$ is computed to

$$
\mathbf{K}_{n}(\zeta, \eta)=n \sum_{j=0}^{n-1} \frac{(n \zeta \bar{\eta})^{j}}{j !} e^{-n \frac{|\zeta|^{2}+|\eta|^{2}}{2}}
$$


Now rescale according to

$$
\zeta=1+z / \sqrt{n}, \quad \eta=1+w / \sqrt{n} .
$$

and note that the rescaled process $\Theta_{n}$ has correlation kernel $K_{n}(z, w)=\frac{1}{n} \mathbf{K}_{n}(\zeta, \eta)$. Using (2.1), we write $K_{n}$ in the form

$$
K_{n}(z, w)=\sum_{j=0}^{n-1}\left(\frac{n \zeta \bar{\eta}}{\lambda}\right)^{j} \frac{\lambda^{j}}{j !} e^{-\lambda}
$$

where

$$
\lambda=\lambda(n)=\frac{n}{2}\left(|\zeta|^{2}+|\eta|^{2}\right)=n+\sqrt{n} \operatorname{Re}(z+w)+\frac{1}{2}\left(|z|^{2}+|w|^{2}\right) .
$$

We next let $X_{n}$ be a Poisson distributed random variable with intensity $\lambda=\lambda(n)$ (in short: $X_{n} \sim \operatorname{Po}(\lambda)$ ), i.e.,

$$
\mathbb{P}\left\{X_{n}=k\right\}=\frac{\lambda^{k}}{k !} e^{-\lambda}, \quad k=0,1, \ldots
$$

We then have the identity

$$
K_{n}(z, w)=\mathbb{E}\left[\left(\frac{n \zeta \bar{\eta}}{\lambda}\right)^{X_{n}} \cdot \mathbf{1}_{\left\{X_{n}<n\right\}}\right]
$$

Now introduce a new random variable $Y_{n}$ by

$$
X_{n}=\lambda+\sqrt{\lambda} Y_{n}
$$

By the central limit theorem, $Y_{n}$ converges in distribution to the standard normal as $n \rightarrow \infty$; the convergence is moreover uniform. (This is the well-known "normal approximation of the Poisson distribution"; uniform convergence follows, e.g., by the Berry-Esseen theorem.)

Now factorize $K_{n}(z, w)$ in the following way:

$$
K_{n}(z, w)=A_{n} \cdot B_{n}:=\left(\frac{n \zeta \bar{\eta}}{\lambda}\right)^{\lambda} \cdot \mathbb{E}\left[\left(\frac{n \zeta \bar{\eta}}{\lambda}\right)^{\sqrt{\lambda} \cdot Y_{n}} \cdot \mathbf{1}_{\left\{Y_{n}<\alpha_{n}\right\}}\right],
$$

where $\alpha_{n}=(n-\lambda) / \sqrt{\lambda}$. Note that $\alpha_{n} \rightarrow-\operatorname{Re}(z+w)$ as $n \rightarrow \infty$.

Lemma 2.2 We have the convergence

$$
B_{n} \rightarrow e^{-b^{2} / 2} F(z+\bar{w}) \text { as } n \rightarrow \infty,
$$


where $b=b(z, w)=\operatorname{Im}(z+\bar{w})$ and $F$ is the free boundary kernel (1.9). Moreover,

$$
A_{n}=e^{i b \sqrt{n}} e^{b^{2} / 2} G(z, w)(1+o(1))
$$

where $G$ is the Ginibre kernel and $o(1) \rightarrow 0$ uniformly on compact sets as $n \rightarrow \infty$.

Proof By a straightforward calculation, we have

$$
\frac{n \zeta \bar{\eta}}{\lambda}=1+\frac{i}{\sqrt{n}} \operatorname{Im}(z+\bar{w})+\frac{1}{n} a+O\left(n^{-3 / 2}\right)
$$

where

$$
a=a(z, w)=z \bar{w}-(z+\bar{w}) \operatorname{Re}(z+w)-\frac{1}{2}\left(|z|^{2}+|w|^{2}\right)+[\operatorname{Re}(z+w)]^{2}
$$

Inserting these expressions into $B_{n}$ (see (2.3)) using the fact that the $Y_{n}$ are asymptotically normal, we now approximate as follows (the symbol " " stands for asymptotic equality as $n \rightarrow \infty)$ :

$$
\begin{aligned}
B_{n} & \sim \frac{1}{\sqrt{2 \pi}} \int_{-\infty}^{\alpha_{n}}\left(1+\frac{i}{\sqrt{n}} \operatorname{Im}(z+\bar{w})+O(1 / n)\right)^{\sqrt{n} \cdot t} e^{-t^{2} / 2} d t \\
& \sim \frac{1}{\sqrt{2 \pi}} \int_{-\infty}^{-\operatorname{Re}(z+\bar{w})} e^{i \operatorname{Im}(z+\bar{w}) t} e^{-t^{2} / 2} d t=e^{-b^{2} / 2} \cdot F(z+\bar{w})
\end{aligned}
$$

We now turn to the factor $A_{n}$ in (2.3). To deal with it, we write $c=\operatorname{Re}(z+w)$. By (2.4), we then have

$$
A_{n}=\left(1+\frac{i b}{\sqrt{n}}+\frac{a}{n}+O\left(n^{-3 / 2}\right)\right)^{n+c \sqrt{n}+O(1)}=e^{i b \sqrt{n}} e^{b^{2} / 2} e^{i b c+a} e^{o(1)}
$$

Noting that $i b c+a=z \bar{w}-\frac{1}{2}\left(|z|^{2}+|w|^{2}\right)$, we finish the proof of the lemma.

By the lemma and relation (2.3), we have

$$
K_{n}(z, w)=e^{i \sqrt{n} \operatorname{Im}(z-w)} K(z, w)(1+o(1)),
$$

where $K$ is the free boundary kernel defined in (0.2). Since the factor $c_{n}(z, w)=$ $e^{i \sqrt{n} \operatorname{Im}(z-w)}$ is a cocycle, this factor can be dropped when computing intensity functions $R_{n, k}(z)=\operatorname{det}\left(K_{n}\left(z_{i}, z_{j}\right)\right)$. This proves the desired convergence of intensity functions, at the same time establishing existence and uniqueness of the boundary Ginibre point field. The proof of Theorem 2.1 is complete. 


\subsection{Hard Edge Ginibre Ensemble}

Let $Q^{S}(z)=|z|^{2}$ when $|z| \leq 1$ and $Q^{S}=+\infty$ otherwise. Let $\left\{\zeta_{i}\right\}_{1}^{n}$ denote a random configuration from the corresponding ensemble. Rescaling about $p=1$ via $z_{j}=\sqrt{n}\left(\zeta_{j}-1\right)$, we obtain a process $\Theta_{n}$. Let $H$ be the hard edge function (0.4).

Theorem 2.3 There exists a unique point field $B G_{h}$ with correlation kernel

$$
K(z, w)=G(z, w) H(z+\bar{w})\left(\mathbf{1}_{\mathbb{L}} \otimes \mathbf{1}_{\mathbb{L}}\right)(z, w) .
$$

The processes $\Theta_{n}$ converge to $B G_{h}$ in the sense that all intensity functions converge locally boundedly almost everywhere, and locally uniformly in $\mathbb{L}^{2}$, to the intensity functions of $B G_{h}$.

Note that $K(z, z)<2$ for all $z$. As we mentioned in Subsection 1.2, the convergence of intensity functions in the theorem implies the existence and uniqueness of a field $B G_{h}$ with correlation kernel $K$. It thus suffices to prove convergence.

By (1.3) and a calculation, a correlation kernel for the hard edge Ginibre process is given by

$$
\mathbf{K}_{n}(\zeta, \eta)=n \sum_{j=0}^{n-1} \frac{(n \zeta \bar{\eta})^{j}}{\gamma(j+1, n)} e^{-n Q^{S}(\zeta) / 2-n Q^{S}(\eta) / 2}
$$

where $\gamma(j+1, n)=\int_{0}^{n} s^{j} e^{-s} d s$ is the lower incomplete Gamma function. Now rescale by

$$
\zeta=1+z / \sqrt{n}, \quad \eta=1+w / \sqrt{n}, \quad z, w \in \mathbb{L},
$$

and write

$$
K_{n}(z, w)=\frac{1}{n} \mathbf{K}_{n}(\zeta, \eta)=\sum_{j=0}^{n-1}\left(\frac{n \zeta \bar{\eta}}{\lambda}\right)^{j} \frac{\lambda^{j}}{\gamma(j+1, n)} e^{-\lambda}
$$

where $\lambda=n\left(|\zeta|^{2}+|\eta|^{2}\right) / 2$ is as in (2.2). Now recall that

$$
\gamma(j+1, n)=j !\left(1-e^{-n} \sum_{k=0}^{j} \frac{n^{k}}{k !}\right)=j ! \mathbb{P}\left(U_{n}>j\right),
$$

where $U_{n} \sim \operatorname{Po}(n)$. By normal approximation of the Poisson distribution,

$$
\mathbb{P}\left(U_{n}>j\right)=\varphi\left(\xi_{j, n}\right)(1+o(1)),
$$


where $\varphi(\xi)=\frac{1}{\sqrt{2 \pi}} \int_{\xi}^{\infty} e^{-t^{2} / 2} d t, \xi_{j, n}=(j-n) / \sqrt{n}$, and $o(1) \rightarrow 0$ as $n \rightarrow \infty$ uniformly in $j$. We have shown that

$$
K_{n}(z, w)=(1+o(1)) \sum_{j=0}^{n-1}\left(\frac{n \zeta \bar{\eta}}{\lambda}\right)^{j} \frac{\lambda^{j}}{j !} e^{-\lambda} \frac{1}{\varphi\left(\xi_{j, n}\right)}
$$

Finally, if $X_{n} \sim \operatorname{Po}(\lambda)$, we can write the last sum in the form

$$
\sum_{j=0}^{n-1}\left(\frac{n \zeta \bar{\eta}}{\lambda}\right)^{j} \frac{1}{\varphi\left(\xi_{j, n}\right)} \mathbb{P}\left(X_{n}=j\right)=\mathbb{E}\left[\left(\frac{n \zeta \bar{\eta}}{\lambda}\right)^{X_{n}} \cdot \frac{1}{\varphi\left(\frac{X_{n}-n}{\sqrt{n}}\right)} \cdot \mathbf{1}_{\left\{X_{n}<n\right\}}\right]
$$

Defining $Y_{n}$ by $X_{n}=\lambda+\sqrt{\lambda} Y_{n}$, we now get a relation of the form

$$
\begin{aligned}
K_{n}(z, w) & =(1+o(1))\left(\frac{n \zeta \bar{\eta}}{\lambda}\right)^{\lambda} \cdot \mathbb{E}\left[\left(\frac{n \zeta \bar{\eta}}{\lambda}\right)^{\sqrt{\lambda} Y_{n}} \frac{1}{\varphi\left(\sqrt{\lambda / n} Y_{n}-\alpha_{n}\right)} \cdot \mathbf{1}_{\left\{Y_{n}<\alpha_{n}\right\}}\right] \\
& =(1+o(1)) A_{n} \cdot \tilde{B}_{n}, \quad \alpha_{n}=(n-\lambda) / \sqrt{n} .
\end{aligned}
$$

Using the asymptotic identities $\alpha_{n} \sim-\operatorname{Re}(z+w)$ and $\lambda / n \sim 1$, we approximate the factor $\tilde{B}_{n}$ as follows, using the central limit theorem,

$$
\begin{aligned}
\tilde{B}_{n} & \sim \frac{1}{\sqrt{2 \pi}} \int_{-\infty}^{-\operatorname{Re}(z+w)} e^{i \operatorname{Im}(z+\bar{w}) t} \frac{1}{\varphi\left(\sqrt{\lambda / n} t-\alpha_{n}\right)} e^{-t^{2} / 2} d t \\
& \sim e^{-b^{2} / 2} \frac{1}{\sqrt{2 \pi}} \int_{-\infty}^{-\operatorname{Re}(z+w)} \frac{1}{\varphi(\operatorname{Re}(z+\bar{w})+t)} e^{-(t-i \operatorname{Im}(z+\bar{w}))^{2} / 2} d t \\
& =e^{-b^{2} / 2} \frac{1}{\sqrt{2 \pi}} \int_{-\infty}^{0} \frac{1}{\varphi(u)} e^{-(u-(z+\bar{w}))^{2} / 2} d u=e^{-b^{2} / 2} H(z+\bar{w})
\end{aligned}
$$

Using the asymptotics for $A_{n}$ in Lemma 2.2, we now conclude that

$$
K_{n}(z, w)=e^{i \sqrt{n} \operatorname{Im}(z-w)} G(z, w) H(z+\bar{w})(1+o(1)), \quad z, w \in \mathbb{L},
$$

where $o(1) \rightarrow 0$ uniformly on compacts. The first factor is a cocycle. We conclude that if $K(z, w)=G(z, w) H(z+\bar{w}) \mathbf{1}_{\mathbb{L}}(z) \mathbf{1}_{\mathbb{L}}(w)$ is the hard edge kernel, then $K_{n} \rightarrow$ $K$ almost everywhere with locally bounded convergence, finishing the proof of the theorem.

\section{Analyticity and Compactness}

In this section, we prove Theorems 1.1 and 1.2. We start by introducing appropriate notation; after that we will deduce our results using normal families coupled with some theory for reproducing kernels. 


\subsection{General Notation}

For a measurable function $\phi: \mathbb{C} \rightarrow \mathbb{R} \cup\{+\infty\}$, we define $L_{\phi}^{2}$ to be the space of functions normed by

$$
\|f\|_{\phi}^{2}=\int_{\mathbb{C}}|f|^{2} e^{-\phi} d A
$$

When $\phi=0$, we just write $L^{2}$ for $L_{\phi}^{2}$ and denote the norm by $\|\cdot\|$.

We denote by $C$ ("large") and $c$ ("small") various positive unspecified constants (independent of $n$ ) whose exact value can change meaning from time to time.

\subsection{Potentials and Reproducing Kernels}

Fix a neighborhood $\Omega$ of $S$ and a number $\delta_{0}>0$ such that $Q$ is real-analytic and strictly subharmonic in the $2 \delta_{0}$-neighborhood of $\Omega$.

Let $\mathcal{P}_{n}$ be the space of analytic polynomials of degree at most $n-1$; we equip $\mathcal{P}_{n}$ with the norm of $L_{n Q}^{2}$. The corresponding space $\mathcal{W}_{n}$ of weighted polynomials is defined to consist of all functions of the form $f=q e^{-n Q / 2}$, where $q \in \mathcal{P}_{n}$; we regard $\mathcal{W}_{n}$ as a subspace of $L^{2}$ and denote the corresponding orthogonal projections by

$$
\pi_{n}: L_{n Q}^{2} \rightarrow \mathcal{P}_{n} \quad \text { and } \quad \Pi_{n}: L^{2} \rightarrow \mathcal{W}_{n}
$$

We write $\mathbf{k}_{n}$ and $\mathbf{K}_{n}$ for the reproducing kernels of $\mathcal{P}_{n}$ and $\mathcal{W}_{n}$, respectively. Then

$$
\mathbf{K}_{n}(\zeta, \eta)=\mathbf{k}_{n}(\zeta, \eta) e^{-n Q(\zeta) / 2} e^{-n Q(\eta) / 2}
$$

It is easy to see that the assignment

$$
U_{n}: L_{n Q}^{2} \rightarrow L^{2}, \quad f \mapsto f e^{-n Q / 2}
$$

is unitary, maps $\mathcal{P}_{n}$ onto $\mathcal{W}_{n}$, and satisfies $U_{n} \pi_{n}=\Pi_{n} U_{n}$.

\subsection{Bulk Approximations}

Let $A(\zeta, \eta)$ be a Hermitian-analytic function defined in a neighborhood in $\mathbb{C}^{2}$ of the set $X=\{(\zeta, \zeta) ; \zeta \in \Omega\}$ such that

$$
A(\zeta, \zeta)=Q(\zeta), \quad \zeta \in \Omega
$$

Choose $\delta_{0}>0$ small enough that $A(\zeta, \eta)$ is defined and Hermitian-analytic in the set of points whose distance to $X$ is $<2 \delta_{0}$. Call this set $\Lambda$.

We define approximations $\mathbf{k}_{n}^{\#}$ and $\mathbf{K}_{n}^{\#}$ via

$$
\mathbf{k}_{n}^{\#}(\zeta, \eta)=n\left(\partial_{1} \bar{\partial}_{2} A\right)(\zeta, \eta) \cdot e^{n A(\zeta, \eta)}, \quad \mathbf{K}_{n}^{\#}(\zeta, \eta)=\mathbf{k}_{n}^{\#}(\zeta, \eta) e^{-n Q(\zeta) / 2} e^{-n Q(\eta) / 2}
$$




\subsection{Auxiliary Estimates}

We recall first the following simple pointwise- $L^{2}$ estimate.

Lemma 3.1 Suppose that $u$ is analytic in the disc $D:=D\left(p ; c / \varrho_{n}\right),\left(\varrho_{n}=\right.$ $\sqrt{n \Delta Q(p)})$, where $Q$ is $C^{2}$-smooth at $p$. Let $f=u e^{-n Q / 2}$. Then there is a number $C$ depending only on $c$ and $\Delta Q(p)$ such that

$$
|f(p)|^{2} \leq C n \int_{D}|f|^{2} d A
$$

Proof Fix a number $a>1$ and consider the function $F_{n}(z)=f\left(p+z / \varrho_{n}\right) \cdot e^{a|z|^{2} / 2}$. We have $\Delta \log \left|F_{n}(z)\right|^{2} \geq-\Delta Q\left(p+z / \varrho_{n}\right) / \Delta Q(p)+a>0$ for $|z| \leq c$ if $n$ is large enough. Hence $\left|F_{n}\right|^{2}$ is subharmonic in $D$, which implies the desired estimate.

Let $\gamma$ be the minimum of $Q+2 U^{\sigma}$, where $U^{\sigma}(z)=\int_{\mathbb{C}} \log \frac{1}{|z-w|} d \sigma(w)$ is the logarithmic potential of the equilibrium measure $\sigma=\Delta Q \cdot \mathbf{1}_{S} d A$. By the obstacle function corresponding to $Q$, we mean the subharmonic function

$$
\check{Q}(\zeta)=-2 U^{\sigma}(\zeta)+\gamma
$$

It is known (see [32]) that $\check{Q}=Q$ on $S$ while $\breve{Q}$ is harmonic on $S^{c}$ and is of logarithmic increase

$$
\check{Q}(\zeta)=\log |\zeta|^{2}+O(1), \quad(\zeta \rightarrow \infty)
$$

Furthermore, $\check{Q}$ has a Lipschitz continuous gradient on $\mathbb{C}$. This leads to the following basic estimate, the "maximum principle of weighted potential theory."

Lemma 3.2 If $f \in \mathcal{W}_{n}$ and $|f| \leq 1$ on $S$, then $|f| \leq e^{-n(Q-\check{Q}) / 2}$ on $\mathbb{C}$.

Proof If $f=p \cdot e^{-n Q / 2}$, then $\frac{1}{n} \log |p|^{2}$ is a subharmonic minorant of $Q$ that grows no faster than $\log |\zeta|^{2}+$ const. as $\zeta \rightarrow \infty$. It is well known that $\check{Q}(\zeta)$ is the supremum of $f(\zeta)$ where $f$ ranges over the functions having these properties (see, e.g., [32]).

To relate the above lemmas to the one-point function $\mathbf{R}_{n}(\zeta)=\mathbf{K}_{n}(\zeta, \zeta)$, we use a general fact for reproducing kernels and obtain

$$
\mathbf{R}_{n}(\zeta)=\sup \left\{|f(\zeta)|^{2} ; f \in \mathcal{W}_{n},\|f\| \leq 1\right\}
$$

Combining this with Lemma 3.2, we conclude the following basic bound.

Lemma 3.3 There a constant $C=C[Q]$ such that $\mathbf{R}_{n}(\zeta) \leq C n e^{-n(Q-\check{Q})(\zeta)}$. 


\subsection{Convergence of Approximate Kernels}

Let $\left\{\zeta_{j}\right\}_{1}^{n}$ denote the point process corresponding to $Q$. A kernel $K_{n}$ for the rescaled process $\left\{z_{j}\right\}_{1}^{n}$ (about $p=0$ in positive real direction) is given by

$$
K_{n}(z, w)=\frac{1}{n \Delta Q(0)} \mathbf{K}_{n}(\zeta, \eta), \quad(z=\sqrt{n \Delta Q(0)} \cdot \zeta, w=\sqrt{n \Delta Q(0)} \cdot \eta) .
$$

Let $V_{n}$ denote the set of points $(z, w)$ such that $(\zeta, \eta) \in \Lambda$. Note that there is a constant $\rho>0$ depending only on $\Delta Q(0)$ such that

$$
\{(z, w) ;|z| \leq \rho \sqrt{n},|w| \leq \rho \sqrt{n}\} \subset V_{n} .
$$

We define the rescaled bulk approximation $K_{n}^{\#}$ by

$$
K_{n}^{\#}(z, w)=\frac{1}{n \Delta Q(0)} \mathbf{K}_{n}^{\#}(\zeta, \eta) .
$$

Lemma 3.4 We have $K_{n}^{\#}(z, w)=c_{n}(z, w) G(z, w)(1+o(1))$ as $n \rightarrow \infty$, where $c_{n}$ are cocycles on $V_{n}$ and $o(1) \rightarrow 0$ as $n \rightarrow \infty$, uniformly on compact subsets of $\mathbb{C}^{2}$.

Proof Put $\Delta Q(0)=\delta$. Recall that

$$
K_{n}^{\#}(z, w)=\frac{1}{\delta}\left(\partial_{1} \bar{\partial}_{2} A\right)(\zeta, \eta) e^{n[A(\zeta, \eta)-A(\zeta, \zeta) / 2-A(\eta, \eta) / 2]} .
$$

By Taylor's formula, the expression in the exponent equals

$$
\begin{aligned}
& i \sqrt{\frac{n}{\delta}} \operatorname{Im}\left\{\partial_{1} A(0,0)(z-w)\right\}+\frac{i}{2 \delta} \operatorname{Im}\left\{\partial_{1}^{2} A(0,0)\left(z^{2}-w^{2}\right)\right\} \\
& \quad+z \bar{w}-|z|^{2} / 2-|w|^{2} / 2
\end{aligned}
$$

up to $1 / \sqrt{n} O\left(\|(z, w)\|^{3}\right)$. The first two terms correspond to cocycles.

Remark The proof of the lemma shows that if $|\zeta|<\delta_{n}$, where $n \delta_{n}^{3} \rightarrow 0$ as $n \rightarrow \infty$, then

$$
n \operatorname{Re} A(\zeta, 0)-n Q(\zeta) / 2-n Q(0) / 2=-n \Delta Q(0)|\zeta|^{2} / 2+o(1)
$$

\subsection{Compactness}

In the proof of Theorem 1.1, we will use the function $\Psi_{n}$ defined on $V_{n}$ by

$$
K_{n}=\Psi_{n} K_{n}^{\#}
$$


Lemma 3.5 The function $\Psi_{n}$ is Hermitian-analytic in the set $V_{n}$.

Proof For $(z, w) \in V_{n}$, we have $(\zeta, \eta) \in \Lambda$ and

$$
\Psi_{n}(z, w)=\frac{K_{n}(z, w)}{K_{n}^{\#}(z, w)}=\frac{\mathbf{K}_{n}(\zeta, \eta)}{\mathbf{K}_{n}^{\#}(\zeta, \eta)},
$$

whence

$$
\Psi_{n}(z, w)=\frac{\mathbf{k}_{n}(\zeta, \eta) e^{-n Q(\zeta) / 2} e^{-n Q(\eta) / 2}}{\left(\partial_{1} \bar{\partial}_{2} A\right)(\zeta, \eta) \cdot n e^{n(A(\zeta, \eta)-Q(\zeta) / 2-Q(\eta) / 2)}}=\frac{\mathbf{k}_{n}(\zeta, \eta)}{\left(\partial_{1} \bar{\partial}_{2} A\right)(\zeta, \eta) \cdot n e^{n A(\zeta, \eta)}} .
$$

The statement follows since $\zeta$ and $\eta$ depend analytically on $z$ and $w$.

Lemma 3.6 For each compact set $K \subset \mathbb{C}^{2}$, there is a constant $C=C_{K}$ such that $\left|\Psi_{n}(z, w)\right|^{2} \leq C e^{|z-w|^{2}}$ when $(z, w) \in K$ and $n$ is large enough.

Proof Choose $n_{0}$ large enough that $K \subset V_{n_{0}}$. Since $K_{n}$ is a positive kernel, and since (by Lemma 3.4) $\left|K_{n}^{\#}(z, w)\right| \rightarrow|G(z, w)|$ uniformly on compact subsets, we have uniformly on $K$,

$$
\left|\Psi_{n}(z, w)\right|^{2}=\left|\frac{K_{n}(z, w)}{K_{n}^{\#}(z, w)}\right|^{2} \leq C \frac{R_{n}(z) R_{n}(w)}{|G(z, w)|^{2}}, \quad\left(R_{n}(z)=K_{n}(z, z)\right),
$$

for all $n \geq n_{0}$. To finish the proof, we note, by Lemma 3.3, that we have a uniform bound $R_{n} \leq C$ and that $|G(z, w)|^{2}=e^{-|z-w|^{2}}$.

Proof of Theorem 1.1 Lemma 3.6 shows that the family $\left\{\Psi_{n}\right\}$ is locally bounded on $\mathbb{C}^{2}$, viz. is a normal family. Pick a locally uniformly convergent subsequence $\left\{\Psi_{n_{k}}\right\}$ converging to a limit $\Psi$. Also fix $z$ and recall that $\int\left|K_{n_{k}}(z, w)\right|^{2} d A(w)=K_{n_{k}}(z, z)$. In terms of the functions $\Psi_{n}$,

$$
\int_{D\left(0 ; \rho \sqrt{n_{k}}\right)}\left|K_{n_{k}}^{\#}(z, w) \cdot \Psi_{n_{k}}(z, w)\right|^{2} d A(w)=\Psi_{n_{k}}(z, z)(1+o(1)), \quad(k \rightarrow \infty),
$$

where $\rho>0$ is the constant in (3.1). Letting $k \rightarrow \infty$, we get, by Fatou's lemma, that the mass-one inequality (1.6) holds.

Finally we use Lemma 3.4 to select cocycles $c_{n}$ such that $c_{n} K_{n}^{\#} \rightarrow G$ uniformly on compact subsets of $\mathbb{C}^{2}$. Then $c_{n_{k}} K_{n_{k}}=\Psi_{n_{k}} \cdot c_{n_{k}} K_{n_{k}}^{\#} \rightarrow G \Psi=K$, finishing the proof of the theorem.

We have shown above that the mass-one inequality (part (i) of Theorem 1.2) holds; i.e.,

$$
\int e^{-|z-w|^{2}}|\Psi(z, w)|^{2} d A(w) \leq R(z)
$$

It will be useful to reformulate this inequality. 
Lemma 3.7 The mass-one inequality holds if and only if

$$
\sum_{n=0}^{\infty} \frac{\left|\partial^{n} R\right|^{2}}{n !} \leq R .
$$

Proof Since $R(z)=\Psi(z, z)$, we have

$$
\begin{aligned}
& \Psi(z+w, z)=\sum_{n=0}^{\infty} \frac{\partial_{1}^{n} \Psi(z, z)}{n !} w^{n}=\sum_{n=0}^{\infty} \frac{\partial^{n} R(z)}{n !} w^{n} \text { and } \\
& \Psi(z, z+w)=\sum_{n=0}^{\infty} \frac{\bar{\partial}^{n} R(z)}{n !} \bar{w}^{n} .
\end{aligned}
$$

It follows that

$$
\begin{gathered}
\int e^{-|w|^{2}}|\Psi(z, z+w)|^{2} d A(w)=\lim _{M \rightarrow \infty} \int_{|w|<M} e^{-|w|^{2}}|\Psi(z, z+w)|^{2} d A(w) \\
=\lim _{M \rightarrow \infty} \sum_{n=0}^{\infty} \frac{\left|\partial^{n} R(z)\right|^{2}}{(n !)^{2}} \int_{|w|<M}|w|^{2 n} e^{-|w|^{2}} d A(w)=\sum_{n=0}^{\infty} \frac{\left|\partial^{n} R(z)\right|^{2}}{n !} .
\end{gathered}
$$

The proof of the lemma is finished.

Remark The proof of Lemma 3.7 shows that the mass-one equation for a kernel $\Psi(z, w)$ is equivalent to that the function $R(z)=\Psi(z, z)$ satisfies

$$
R=\sum_{n=0}^{\infty} \frac{\left|\partial^{n} R\right|^{2}}{n !}
$$

One can regard this as a differential equation of infinite order.

\subsection{Holomorphic Kernels and Positivity}

In this subsection, we prove Theorem 1.2. Part (i), the mass-one inequality, is already explained in the proof of Theorem 1.1.

It is convenient to prove first the corresponding properties for the holomorphic kernel

$$
L(z, w):=e^{z \bar{w}} \Psi(z, w)
$$

We shall find that $L$ is the reproducing kernel for a certain Hilbert space $\mathcal{H}_{*}$ of entire functions that is contractively embedded in the Fock space $L_{a}^{2}(\mu)$ of entire functions square-integrable with respect to the measure

$$
d \mu(z)=e^{-|z|^{2}} d A(z)
$$


We will also prove that the complementary holomorphic kernel $\tilde{L}(z, w):=e^{z \bar{w}}(1-$ $\Psi(z, w))$ is a positive kernel. Our proof of the latter fact depends on a theorem of Aronszajn on differences of reproducing kernels.

To set things up, suppose that we rescale about the boundary point $p=0$ in the positive real direction. Suppose also that $\Delta Q(0)=1$. The rescaling is then simply

$$
z=\sqrt{n} \zeta, \quad w=\sqrt{n} \eta, \quad \text { etc. }
$$

Let $A(\zeta, \eta)$ be the Hermitian-analytic function defined in a neighborhood of the diagonal such that $A(\zeta, \zeta)=Q(\zeta)$. Recall (cf. Section 3) that, along some subsequence, we have $\Psi=\lim \Psi_{n}$, where

$$
\Psi_{n}(z, w):=\frac{K_{n}(z, w)}{K_{n}^{\#}(z, w)}=\frac{\mathbf{k}_{n}(\zeta, \eta)}{n\left(\partial_{1} \bar{\partial}_{2} A\right)(\zeta, \eta) e^{n A(\zeta, \eta)}} .
$$

By Taylor's formula, there is $\delta>0$ such that

$$
A(\zeta, \eta)=\zeta \bar{\eta}+f(\zeta, \eta)+H(\zeta)+\bar{H}(\eta), \quad|\zeta|<\delta,|\eta|<\delta
$$

where

$$
H(\zeta)=\sum_{j=0}^{2} \frac{\partial_{1}^{j} A(0,0)}{j !} \zeta^{j}, \quad f(\zeta, \eta)=\sum_{j+k \geq 3}\left(\partial_{1}^{j} \bar{\partial}_{2}^{k} A\right)(0,0) \frac{\zeta^{j}}{j !} \frac{\bar{\eta}^{k}}{k !}
$$

Let us extend $f$ to a smooth function on $\mathbb{C}^{2}$ in some way. We will require that the extended function satisfies $f(\zeta, \zeta)=Q(\zeta)-|\zeta|^{2}-2 \operatorname{Re} H(\zeta)$ for all $\zeta \in \mathbb{C}$. (The function $H$ is of course well-defined everywhere, being a second-degree polynomial.) It is important to observe that $f(\zeta, \zeta)=O\left(|\zeta|^{3}\right)$ as $\zeta \rightarrow 0$.

Put

$$
f_{n}(z, w):=n f(z / \sqrt{n}, w / \sqrt{n}), \quad H_{n}(z):=n H(z / \sqrt{n}) .
$$

We extend the function $A_{n}(z, w)=n A(\zeta, \eta)$ to $\mathbb{C}^{2}$ by

$$
A_{n}(z, w)=z \bar{w}+f_{n}(z, w)+H_{n}(z)+\bar{H}_{n}(w) .
$$

Now define

$$
E_{n}(z, w):=e^{z \bar{w}+f_{n}(z, w)}
$$

and

$$
L_{n}(z, w):=\left(\partial_{1} \bar{\partial}_{2} A_{n}\right)(z, w) \cdot E_{n}(z, w) \cdot \Psi_{n}(z, w)
$$


Then by (3.3) and (3.4),

$$
L_{n}(z, w)=\frac{1}{n} \mathbf{k}_{n}\left(\frac{z}{\sqrt{n}}, \frac{w}{\sqrt{n}}\right) e^{-A_{n}(z, w)+z \bar{w}+f_{n}(z, w)}=k_{n}(z, w) e^{-H_{n}(z)-\bar{H}_{n}(w)} .
$$

Next define an $n$-dimensional Hilbert space

$$
\mathcal{H}_{n}=\left\{f=q \cdot e^{-H_{n}} ; q \in \mathcal{P}_{n}\right\}
$$

equipped with the norm of $L^{2}\left(\mu_{n}\right)$, where

$$
d \mu_{n}(z):=e^{-|z|^{2}-f_{n}(z, z)} d A(z) .
$$

Note that $\mathcal{H}_{n}$ consists of entire functions. Finally recall that $L(z, w)=e^{z \bar{w}} \Psi(z, w)$.

Lemma 3.8 $L_{n}$ is the reproducing kernel for $\mathcal{H}_{n}$ and $L_{n} \rightarrow L$ locally uniformly on $\mathbb{C}^{2}$ as $n \rightarrow \infty$. Moreover, for all $z \in \mathbb{C}$, we have $\int_{\mathbb{C}}|L(z, w)|^{2} e^{-|w|^{2}} d A(w)<\infty$.

Proof To show that $L_{n} \rightarrow L$ locally uniformly, it suffices to note that

$$
E_{n}(z, w)=e^{z \bar{w}+n f(z / \sqrt{n}, w / \sqrt{n})}=e^{z \bar{w}+O\left(n^{-1 / 2}\right)}, \quad(n \rightarrow \infty),
$$

where the $O$-constant is uniform on compact subsets of $\mathbb{C}^{2}$. Moreover, the mass-one inequality shows that

$$
\begin{aligned}
& \int_{\mathbb{C}}|L(z, w)|^{2} e^{-|w|^{2}} d A(w)=e^{|z|^{2}} \int_{\mathbb{C}} e^{-|z-w|^{2}}|\Psi(z, w)|^{2} d A(w) \\
& \quad \leq e^{|z|^{2}} \Psi(z, z) .
\end{aligned}
$$

It remains to show that $L_{n}$ has the reproducing property stated above. Write $L_{n, w}(z)=L_{n}(z, w)$. For an element $f=q \cdot e^{-H_{n}}$ of $\mathcal{H}_{n}$, we then have

$$
\begin{aligned}
\left\langle f, L_{n, w}\right\rangle_{\mathcal{H}_{n}} & =\int_{\mathbb{C}} q(z) e^{-H_{n}(z)} \bar{L}_{n}(z, w) e^{-|z|^{2}-f_{n}(z, z)} d A(z) \\
& =e^{-H_{n}(w)} \int_{\mathbb{C}} q(z) \bar{k}_{n}(z, w) e^{-H_{n}(z)-\bar{H}_{n}(z)-|z|^{2}-f_{n}(z, z)} d A(z) .
\end{aligned}
$$

The expression in the exponent equals $-Q_{n}(z):=-n Q(z / \sqrt{n})$. Writing $\tilde{q}(\zeta)=q(z)$ and recalling that $\mathbf{k}_{n}$ is the reproducing kernel for the subspace $\mathcal{P}_{n}$ of polynomials of the space $L_{n Q}^{2}$ normed by $\|p\|_{n Q}^{2}:=\int|p|^{2} e^{-n Q} d A$, we now find

$$
\left\langle f, L_{n, w}\right\rangle_{\mathcal{H}_{n}}=e^{-H_{n}(w)}\left\langle\tilde{q}, \mathbf{k}_{n, \eta}\right\rangle_{n Q}=e^{-H_{n}(w)} \tilde{q}(\eta)=e^{-H_{n}(w)} q(w)=f(w)
$$

Also $L_{n, w}$ belongs to the space $\mathcal{H}_{n}$ since $L_{n, w}(z)=C_{w} k_{n, w}(z) e^{-H_{n}(z)}$, where $C_{w}=$ $e^{-\bar{H}_{n}(w)}$. 
We can now finish the proof of part (ii) of Theorem 1.2.

Let $\mathcal{M}$ be the algebraic linear span of the kernels $L_{z}, z \in \mathbb{C}$, with semi-definite inner product $\left\langle L_{z}, L_{w}\right\rangle_{*}:=L(w, z)$. By the zero-one law, we can assume that $L(z, z)>0$ for all $z$, so the inner product is actually a true (positive definite) inner product. By Fatou's lemma and the convergence $L_{n} \rightarrow L$, we now derive a basic inequality (where $\left.d \mu(z)=e^{-|z|^{2}} d A(z)\right)$ :

$$
\begin{aligned}
\left\|\sum_{j=1}^{N} \alpha_{j} L_{z_{j}}\right\|_{L^{2}(\mu)}^{2} & \leq \liminf _{n \rightarrow \infty} \sum_{j, k=1}^{N} \alpha_{j} \bar{\alpha}_{k} \int_{\mathbb{C}} L_{n}\left(w, z_{j}\right) \bar{L}_{n}\left(w, z_{k}\right) d \mu_{n}(w) \\
& =\liminf _{n \rightarrow \infty} \sum_{j, k=1}^{N} \alpha_{j} \bar{\alpha}_{k} L_{n}\left(z_{j}, z_{k}\right)=\sum_{j, k=1}^{N} \alpha_{j} \bar{\alpha}_{k} L\left(z_{j}, z_{k}\right) \\
& =\left\|\sum_{j=1}^{N} \alpha_{j} L_{z_{j}}\right\|_{*}^{2},
\end{aligned}
$$

so $\mathcal{M}$ is contained in $L^{2}(\mu)$ and the inclusion $I: \mathcal{M} \rightarrow L^{2}(\mu)$ is a contraction.

It follows that the completion $\mathcal{H}_{*}$ of $\mathcal{M}$ can be regarded as a (possibly non-closed) subspace of $L_{a}^{2}(\mu)$. We will write $\mathcal{H}_{\Psi}$ for $\mathcal{H}_{*}$ and speak of the space of entire functions associated with the kernel $L(z, w)=e^{z \bar{w}} \Psi(z, w)$. (The mass-one equation is equivalent to that the inclusion $I$ be isometric; see Subsection 8.2.)

Now we finish the proof of part (iii) of Theorem 1.2. Note that the Fock space $L_{a}^{2}(\mu)$ has reproducing kernel $L_{0}(z, w)=e^{z \bar{w}}$. Let us define a Hermitian entire function $\tilde{L}$ by $\tilde{L}=L_{0}-L$; i.e.,

$$
\tilde{L}(z, w):=e^{z \bar{w}}(1-\Psi(z, w)) .
$$

Since the inclusion $I: \mathcal{H}_{*} \rightarrow L_{a}^{2}(\mu)$ is contractive, we can apply a theorem of Aronszajn [9, Theorem II, p. 355], which implies that the corresponding reproducing kernels then satisfy that the difference $L_{0}-L$ is a positive kernel. Hence the kernel $\tilde{K}=G(1-\Psi)$ is a positive kernel as well, since $\tilde{K}(z, w)=\tilde{L}(z, w) e^{-|z|^{2} / 2-|w|^{2} / 2}$. The proof of Theorem 1.2 part (iii) is complete.

\section{Ward's Equation and the Mass-One Inequality}

In this section, we prove part (ii) of Theorem 1.3. We start by deriving a slightly modified (or "localized,") form of the Ward identity used in [3]. This modification is necessary when dealing with hard edge processes and is in general quite convenient.

The main theme of the section is the rescaled form of this identity and the passage to subsequential limits. In the process of proving this convergence, we will for instance verify the zero one-law for limiting one-point functions. 


\subsection{Ward's Identity}

To set things up, fix a test function $\psi \in C_{0}^{\infty}(\mathbb{C})$. Define a function $W_{n}^{+}[\psi]$ of $n$ variables by

$$
W_{n}^{+}[\psi]=\mathrm{I}_{n}[\psi]-\mathrm{II}_{n}[\psi]+\mathrm{III}_{n}[\psi]
$$

where

$$
\begin{aligned}
\mathrm{I}_{n}[\psi](\zeta) & =\frac{1}{2} \sum_{j \neq k}^{n} \frac{\psi\left(\zeta_{j}\right)-\psi\left(\zeta_{k}\right)}{\zeta_{j}-\zeta_{k}} ; \mathrm{II}_{n}[\psi](\zeta)=n \sum_{j=1}^{n}[\partial Q \cdot \psi]\left(\zeta_{j}\right) ; \\
\mathrm{III}_{n}[\psi](\zeta) & =\sum_{j=1}^{n} \partial \psi\left(\zeta_{j}\right) .
\end{aligned}
$$

Here $\left(\zeta_{j}\right)_{1}^{n}$ is picked randomly with respect to the Boltzmann-Gibbs law $\mathbb{P}_{n}$, see (1.1).

We assume only that $Q$ is smooth in a neighborhood of the support of $\psi$. We can then make sense of $W_{n}^{+}[\psi]$ even though $\partial Q$ may be undefined in portions of the plane. Indeed, we define

$$
[\partial Q \cdot \psi](\zeta)=\left\{\begin{array}{cc}
\partial Q(\zeta) \cdot \psi(\zeta) & \text { if } \quad \zeta \in \operatorname{supp} \psi \\
0 & \text { otherwise }
\end{array}\right.
$$

We then have the following form of Ward's identity.

Theorem 4.1 $\mathbb{E}_{n} W_{n}^{+}[\psi]=0$.

Proof We modify the argument in [3]. Given $\zeta \in \mathbb{C}$ and $\varepsilon>0$, we let $D_{\varepsilon \psi}(\zeta)$ be the closed disc centered at $\zeta$ of radius $\varepsilon|\psi(\zeta)|$. Choosing $\varepsilon=\varepsilon(\psi)>0$ sufficiently small, there are two alternatives for each point $\zeta \in \mathbb{C}$ : (i) $Q$ is $C^{2}$-smooth in a neighborhood $D_{\varepsilon \psi}(\zeta)$ of $\zeta$, or (ii) $\psi(\zeta)=0$.

Now fix an arbitrary sequence $\zeta=\left(\zeta_{j}\right)_{1}^{n}$ and put $\eta_{j}=\phi\left(\zeta_{j}\right)=\zeta_{j}+\varepsilon \psi\left(\zeta_{j}\right) / 2$, $1 \leq j \leq n$. The Jacobian for $\phi$ is $J(\omega)=|\partial \phi(\omega)|^{2}-|\bar{\partial} \phi(\omega)|^{2}=1+\varepsilon \operatorname{Re} \partial \psi(\omega)+$ $O\left(\varepsilon^{2}\right),(\varepsilon \rightarrow 0)$, whence (with $\left.\mathrm{III}_{n}=\mathrm{III}_{n}[\psi]\right)$

$$
d V_{n}(\eta)=\prod_{j=1}^{n}\left|J\left(\zeta_{j}\right)\right| d A\left(\zeta_{j}\right)=\left[1+\varepsilon \operatorname{Re} \operatorname{III}_{n}(\zeta)+O\left(\varepsilon^{2}\right)\right] d V_{n}(\zeta)
$$

Moreover,

$$
\sum_{j \neq k}^{n} \log \left|\eta_{j}-\eta_{k}\right|^{-1}=\sum_{j \neq k}^{n} \log \left|\zeta_{j}-\zeta_{k}\right|^{-1}-\varepsilon \operatorname{Re} \mathrm{I}_{n}(\zeta)+O\left(\varepsilon^{2}\right), \quad(\varepsilon \rightarrow 0) .
$$

If $D_{\varepsilon \psi}\left(\zeta_{j}\right)$ is contained in a domain where $Q$ is $C^{2}$-smooth, then, by Taylor's formula,

$$
Q\left(\eta_{j}\right)=Q\left(\zeta_{j}+\frac{\varepsilon}{2} \psi\left(\zeta_{j}\right)\right)=Q\left(\zeta_{j}\right)+\varepsilon \operatorname{Re}[\partial Q \cdot \psi]\left(\zeta_{j}\right)+O\left(\varepsilon^{2}\right) .
$$


For other $j$ 's we have $\psi\left(\zeta_{j}\right)=0$ and $\eta_{j}=\zeta_{j}$, whence (4.2) holds, since $[\partial Q \cdot \psi]\left(\zeta_{j}\right)=0$ by definition. Hence (4.2) holds in all cases, so

$$
n \sum_{j=1}^{n} Q\left(\eta_{j}\right)=n \sum_{j=1}^{n} Q\left(\zeta_{j}\right)+\varepsilon \operatorname{Re} \mathrm{II}_{n}(\zeta)+O\left(\varepsilon^{2}\right)
$$

Now (4.1) and (4.3) imply that the Hamiltonian (1.2) satisfies

$$
H_{n}(\eta)=H_{n}(\zeta)+\varepsilon \cdot \operatorname{Re}\left(-\mathrm{I}_{n}(\zeta)+\mathrm{II}_{n}(\zeta)\right)+O\left(\varepsilon^{2}\right)
$$

It follows that the partition function $Z_{n}:=\int_{\mathbb{C}^{n}} e^{-H_{n}(\eta)} d V_{n}(\eta)$ satisfies

$$
Z_{n}=\int_{\mathbb{C}^{n}} e^{-H_{n}(\zeta)-\varepsilon \operatorname{Re}\left(-\mathrm{I}_{n}(\zeta)+\mathrm{II}_{n}(\zeta)\right)+O\left(\varepsilon^{2}\right)}\left[1+\varepsilon \operatorname{Re} \operatorname{III}_{n}(\zeta)+O\left(\varepsilon^{2}\right)\right] d V_{n}(\zeta)
$$

Since the integral is independent of $\varepsilon$, the coefficient of $\varepsilon$ in the right-hand side must vanish; i.e.,

$$
\operatorname{Re} \int_{\mathbb{C}^{n}}\left(\mathrm{III}_{n}(\zeta)+\mathrm{I}_{n}(\zeta)-\mathrm{II}_{n}(\zeta)\right) e^{-H_{n}(\zeta)} d V_{n}(\zeta)=0
$$

or $\operatorname{Re} \mathbb{E}_{n} W_{n}^{+}[\psi]=0$. Replacing $\psi$ by $i \psi$ in the preceding argument gives $\operatorname{Im} \mathbb{E}_{n} W_{n}^{+}[\psi]=0$, and the theorem follows.

\subsection{Rescaled Version}

We now fix a point $p$ in a small neighborhood $\Omega$ of $S$, where $Q$ is $C^{2}$-smooth. We rescale the system $\left\{\zeta_{j}\right\}_{1}^{n}$ about $p$ in the usual way, obtaining the rescaled system $\Theta_{n}=\left\{z_{j}\right\}_{1}^{n}$, where $z_{j}=e^{-i \theta} \sqrt{n \Delta Q(p)}\left(\zeta_{j}-p\right)$. The Berezin kernel rooted at $p$ is defined by

$$
B_{n}(z, w)=\frac{R_{n, 1}(z) R_{n, 1}(w)-R_{n, 2}(z, w)}{R_{n, 1}(z)},
$$

where $R_{n, k}$ are the rescaled intensity functions, see (1.5).

Theorem 4.2 We have

$$
\bar{\partial} C_{n}(z)=R_{n}(z)-1-\Delta \log R_{n}(z)+o(1), \quad z \in \mathbb{C},
$$

where

$$
C_{n}(z):=\int_{\mathbb{C}} \frac{B_{n}(z, w)}{z-w} d A(w) \text { and } R_{n}(z):=R_{n, 1}(z)=B_{n}(z, z)
$$

and $o(1) \rightarrow 0$ uniformly on compact subsets of $\mathbb{C}$ as $n \rightarrow \infty$. 
Proof We can without loss of generality assume that $p=0$ and $\theta=0$. Fix a test function $\psi$ supported in the dilated set $\sqrt{n \delta} \cdot U$, where $\delta=\Delta Q(0)$. Write

$$
z=\sqrt{n \delta} \cdot \zeta, \quad w=\sqrt{n \delta} \cdot \eta
$$

and let $\psi_{n}(\zeta)=\psi(z)$. Thus supp $\psi_{n} \subset U$. The change of variables $(\zeta, \eta) \mapsto(z, w)$ gives

$$
\begin{aligned}
\mathbb{E}_{n} \mathrm{I}_{n}\left[\psi_{n}\right] & =\frac{1}{2} \iint_{\mathbb{C}^{2}} \frac{\psi(\zeta \sqrt{n \delta})-\psi(\eta \sqrt{n \delta})}{\zeta-\eta} \mathbf{R}_{n, 2}(\zeta, \eta) d V_{2}(\zeta, \eta) \\
& =\sqrt{n \delta} \frac{1}{2} \iint_{\mathbb{C}^{2}} \frac{\psi(z)-\psi(w)}{z-w} R_{n, 2}(z, w) d V_{2}(z, w) \\
& =\sqrt{n \delta} \int_{\sqrt{n \delta} \cdot U} \psi(z) d A(z) \int_{\mathbb{C}} \frac{R_{n, 2}(z, w)}{z-w} d A(w) .
\end{aligned}
$$

Similarly, since supp $\psi_{n} \subset U$,

$$
\begin{aligned}
\mathbb{E}_{n} \mathrm{II}_{n}\left[\psi_{n}\right] & =n \int_{U}\left[\partial Q \cdot \psi_{n}\right](\zeta) \mathbf{R}_{n, 1}(\zeta) d A(\zeta) \\
& =n \int_{\sqrt{n \delta} \cdot U} \partial Q\left(\frac{z}{\sqrt{n \delta}}\right) \cdot \psi(z) \cdot R_{n, 1}(z) d A(z)
\end{aligned}
$$

Finally, in the sense of distributions,

$$
\begin{aligned}
\mathbb{E}_{n} \operatorname{III}_{n}\left[\psi_{n}\right] & =\int_{U} \partial \psi_{n} \cdot \mathbf{R}_{n, 1} d A=\sqrt{n \delta} \int_{U} \partial \psi(\zeta \sqrt{n \delta}) \mathbf{R}_{n, 1}(\zeta) d A(\zeta) \\
& =\sqrt{n \delta} \int_{\sqrt{n \delta} \cdot U} \partial \psi \cdot R_{n, 1} d A=-\sqrt{n \delta} \int_{\sqrt{n \delta} \cdot U} \psi \cdot \partial R_{n, 1} d A
\end{aligned}
$$

After dividing by $\sqrt{n \delta}$ in Ward's identity (Theorem 4.1), we deduce that

$$
\begin{aligned}
& \int_{\sqrt{n \delta} \cdot U} \psi(z)\left[\int_{\mathbb{C}} \frac{R_{n, 2}(z, w)}{z-w} d A(w)\right] d A(z) \\
& \quad=\int_{\sqrt{n \delta} \cdot U} \frac{\sqrt{n}}{\sqrt{\delta}} \partial Q\left(\frac{z}{\sqrt{n \delta}}\right) \cdot \psi(z) \cdot R_{n, 1}(z) d A(z)+\int_{\sqrt{n \delta} \cdot U} \psi \cdot \partial R_{n, 1} d A .
\end{aligned}
$$

Since $\psi$ is arbitrary, we get the following identity, in the sense of distributions,

$$
\int_{\mathbb{C}} \frac{R_{n, 2}(z, w)}{z-w} d A(w)=\frac{\sqrt{n}}{\sqrt{\delta}} \partial Q\left(\frac{z}{\sqrt{n \delta}}\right) \cdot R_{n, 1}(z)+\partial R_{n, 1}(z), \quad z \in \sqrt{n \delta} \cdot U .
$$

But

$$
\frac{R_{n, 2}(z, w)}{R_{n, 1}(z)}=R_{n, 1}(w)-B_{n}(z, w)
$$


so we can write (4.4) as

$$
\int_{\mathbb{C}} \frac{R_{n, 1}(w)}{z-w} d A(w)-C_{n}(z)=\frac{\sqrt{n}}{\sqrt{\delta}} \partial Q\left(\frac{z}{\sqrt{n \delta}}\right)+\partial \log R_{n, 1}(z) .
$$

Taking a $\bar{\partial}$-derivative now gives

$$
R_{n, 1}(z)-\bar{\partial} C_{n}(z)=\frac{1}{\delta} \Delta Q\left(\frac{z}{\sqrt{n \delta}}\right)+\Delta \log R_{n, 1}(z), \quad z \in \sqrt{n \delta} \cdot U
$$

As $n \rightarrow \infty$, we have that $\Delta Q(z / \sqrt{n \delta}) / \delta \rightarrow 1$ uniformly on compact subsets of $\mathbb{C}$. We have shown

$$
R_{n, 1}(z)-\bar{\partial} C_{n}(z)=1+\Delta \log R_{n, 1}(z)+o(1), \quad z \in \mathbb{C}
$$

Recalling that $R_{n, 1}(z)=B_{n}(z, z)$, we conclude the proof of Theorem 4.2.

\subsection{Ward's Equation}

Let $K=G \Psi$ denote any limiting kernel in Theorem 1.1. Referring to a suitable subsequence, we write

$$
R(z)=\lim _{k \rightarrow \infty} R_{n_{k}}(z)=K(z, z)=\Psi(z, z)
$$

for the one-point function. In the following, we shall assume that $R$ does not vanish identically.

We shall also use the corresponding holomorphic kernel

$$
L(z, w):=e^{z \bar{w}} \Psi(z, w)
$$

and write $L_{z}(w):=L(w, z)$.

Lemma 4.3 $L(z, w)$ is a positive kernel, and $z \mapsto L(z, z)$ is logarithmically subharmonic; i.e., the function $|z|^{2}+\log R(z)$ is subharmonic.

Proof Since $K(z, w)=L(z, w) e^{-|z|^{2} / 2-|w|^{2} / 2}$ is a positive matrix, i.e., $\sum \alpha_{j} \bar{\alpha}_{k}$ $K\left(z_{j}, z_{k}\right) \geq 0$ for all choices of points $z_{j}$ and scalars $\alpha_{j}$, we infer that $\sum \beta_{j} \bar{\beta}_{k} L\left(z_{j}, z_{k}\right) \geq 0$, where $\beta_{j}=\alpha_{j} e^{-\left|z_{j}\right|^{2} / 2}$; i.e., $L$ is a positive kernel. Following Aronszajn [9], we can then define a semi-definite inner product on the span of the $L_{z}$ 's by $\left\langle L_{z}, L_{w}\right\rangle_{*}=L(w, z)$. The completion of the span the functions $L_{z}(z \in \mathbb{C})$ is a semi-normed Hilbert space $\mathcal{H}_{*}$ of entire functions, and the reproducing kernel in this space is $L$.

Since the kernel $L$ is Hermitian-entire, the function $F(z, w)=\left\langle L_{w}, L_{z}\right\rangle_{*}$ is also Hermitian-entire. Moreover, since $\partial_{z} F(z, w)=\left\langle L_{w}, \bar{\partial}_{z} L_{z}\right\rangle_{*}, \bar{\partial}_{w} \partial_{z} F(z, w)=$ 
$\left\langle\bar{\partial}_{w} L_{w}, \bar{\partial}_{z} L_{z}\right\rangle_{*}$, etc., it follows that at points where $L(z, z)>0$, we have

$$
\Delta \log L(z, z)=\frac{\left\|\bar{\partial}_{z} L_{z}\right\|_{*}^{2} \cdot\left\|L_{z}\right\|_{*}^{2}-\left|\left\langle\bar{\partial}_{z} L_{z}, L_{z}\right\rangle_{*}\right|^{2}}{L(z, z)^{2}} \geq 0,
$$

where we used the Cauchy-Schwarz inequality. On the other hand, when $L(z, z)=0$, we have $\log L(z, z)=-\infty$. It follows that $\log L(z, z)$ has the sub-mean value property and hence is subharmonic.

Lemma 4.4 If $R\left(z_{0}\right)=0$, then there is a real-analytic function $\tilde{R}$ such that

$$
R(z)=\left|z-z_{0}\right|^{2} \tilde{R}(z) .
$$

Moreover, if $R$ does not vanish identically, then all zeros of $R$ are isolated.

Proof By Lemma 3.7, we have the mass-one inequality

$$
\sum_{j=0}^{\infty} \frac{\left|\partial^{j} R(z)\right|^{2}}{j !} \leq R(z), \quad(z \in \mathbb{C})
$$

so since $R\left(z_{0}\right)=0$, we have $\partial^{j} R\left(z_{0}\right)=0$ for all $j$. However, $\partial^{j} R\left(z_{0}\right)=\partial_{1}^{j} \Psi\left(z_{0}, z_{0}\right)$ and $\bar{\partial}^{j} R\left(z_{0}\right)=\bar{\partial}_{2}^{j} \Psi\left(z_{0}, z_{0}\right)$, so the Hermitian function $\Psi(z, w)$ vanishes whenever $\left(z-z_{0}\right)\left(w-z_{0}\right)=0$. Hence we can write $\Psi(z, w)=\left(z-z_{0}\right)\left(\bar{w}-\bar{z}_{0}\right) \Psi_{1}(z, w)$, where $\Psi_{1}$ is another Hermitian-entire function. If we define $\tilde{R}(z)=\Psi_{1}(z, z)$, we now have $R(z)=\left|z-z_{0}\right|^{2} \tilde{R}(z)$.

To prove the second statement, assume that the zeros of $R$ have an accumulation point, i.e., that there exists a convergent sequence $\left(z_{j}\right)_{1}^{\infty}$ of distinct zeros of $R$. Fix a point $w$, and put $\psi_{w}(z)=\Psi(z, w)$. By the argument above, we have that $\Psi(z, w)=0$ if $z=z_{j}$, so the holomorphic function $\psi_{w}$ vanishes at all points $z_{j}$, whence $\psi_{w}$ vanishes identically. Since $w$ was arbitrary, $\Psi=0$, and hence $R(z) \equiv \Psi(z, z) \equiv 0$.

Note that Lemma 4.3 says that the distribution $1+\Delta \log R$ is a positive measure.

Lemma 4.5 In the situation of Lemma 4.4, the measure $1+\Delta \log \tilde{R}$ is positive in a neighborhood of $z_{0}$.

Proof Let $z_{0}$ be a zero of $R$, and let $\chi=\mathbf{1}_{D}$ be the characteristic function of some small disc $D=D\left(z_{0} ; \varepsilon\right)$ about $z_{0}$. Put $\mu=\chi \cdot(1+\Delta \log R)$ so $\mu$ is a positive measure by the previous lemma. By Lemma 4.4 , we can write $\mu=\delta_{z_{0}}+\chi \cdot(1+\Delta \log \tilde{R})$, so the function

$$
S(z):=\log \left(e^{|z|^{2}} \tilde{R}(z)\right)
$$

must satisfy that $\Delta S \geq 0$ in the sense of distributions on the punctured disc $D^{\prime}=$ $D \backslash\left\{z_{0}\right\}$. If $\tilde{R}\left(z_{0}\right)>0$, then $S$ extends analytically to $z_{0}$ and is hence subharmonic in $D$. Otherwise $S\left(z_{0}\right)=-\infty$. Then $S$ has the sub-mean value property in $D$. Since $S$ is also upper semicontinuous, $S$ is subharmonic in the entire disc $D$ as desired. 
Write $Z$ for the set of isolated zeros of $R$. When $z \notin Z$, we can write $B(z, w)=$ $|K(z, w)|^{2} / K(z, z)$ and define

$$
C(z)=\int_{\mathbb{C}} \frac{B(z, w)}{z-w} d A(w) .
$$

This clearly defines a smooth function in the complement of $Z$.

Lemma 4.6 $C_{n_{k}}$ converges boundedly and locally uniformly on $Z^{c}$ to $C$ as $k \rightarrow \infty$. In particular, $C$ is uniformly bounded on $Z^{c}$.

Proof Fix a number $\varepsilon$ with $0<\varepsilon<1$. By the locally uniform convergence $c_{n_{k}} K_{n_{k}} \rightarrow$ $K$, we can pick $N$ such that if $k>N,|z|<1 / \varepsilon$, $\operatorname{dist}(z, Z) \geq \varepsilon$, and $|w|<2 / \varepsilon$, then

$$
\left|B_{n_{k}}(z, w)-B(z, w)\right|<\varepsilon^{2} .
$$

For $k>N$ and $z$ with $\operatorname{dist}(z, Z) \geq \varepsilon,|z|<1 / \varepsilon$, it follows that

$$
\begin{aligned}
& \left|C_{n_{k}}(z)-C(z)\right| \leq \int\left|\frac{B_{n_{k}}(z, w)-B(z, w)}{z-w}\right| d A(w) \\
& \quad \leq\left(\int_{|z-w|<1 / \varepsilon}+\int_{|z-w|>1 / \varepsilon}\right)\left|\frac{B_{n_{k}}(z, w)-B(z, w)}{z-w}\right| d A(w) \\
& \quad \leq \varepsilon^{2} \int_{|z-w|<1 / \varepsilon} \frac{1}{|z-w|} d A(w)+\varepsilon \int\left|B_{n_{k}}(z, w)-B(z, w)\right| d A(w) \leq 4 \varepsilon .
\end{aligned}
$$

We have shown that the convergence $C_{n_{k}} \rightarrow C$ is uniform on compact subsets of $Z^{c}$. We next recall the inequalities

$$
B_{n_{k}}(z, w) \leq R_{n_{k}}(w) \leq 1+o\left(n_{k}\right)
$$

where $o\left(n_{k}\right) \rightarrow 0$ uniformly on compacts as $k \rightarrow \infty$. (Note that $R \leq 1$, since the mass-one inequality implies $R-R^{2} \geq 0$ ). It follows that

$$
\begin{aligned}
& \left|C_{n_{k}}(z)\right| \leq\left(1+o\left(n_{k}\right)\right) \int_{|w-z| \leq 1} \frac{1}{|w-z|} d A(w) \\
& +\int_{|w-z|>1} B_{n_{k}}(z, w) d A(w) \leq 3+o\left(n_{k}\right) .
\end{aligned}
$$

This proves the uniform bound $|C(z)| \leq 3$ on the complement of $Z$.

Lemma 4.7 Suppose that $K$ is nontrivial. Then Ward's equation

$$
\bar{\partial} C=R-1-\Delta \log R
$$

holds in the sense of distributions. 
Proof By Theorem 4.2, we know that $\bar{\partial} C_{n}=R_{n}-1-\Delta \log R_{n}+o(1)$, where " $o$ (1)" is some function that converges to 0 uniformly on compacts as $n \rightarrow \infty$. By Lemma 4.6, the functions $C_{n_{k}}$ converge to $C$ boundedly and locally uniformly on $Z^{c}$. Since $Z$ is discrete, this implies that $\int C_{n_{k}} f d A \rightarrow \int C f d A$ for each test function $f$, viz. $C_{n_{k}} \rightarrow C$ in the sense of distributions, and also $\bar{\partial} C_{n_{k}} \rightarrow \bar{\partial} C$ in that sense. It follows that the functions $\Delta \log R_{n_{k}}$ converge in the sense of distributions. Since $R_{n_{k}} \rightarrow R$ locally uniformly, the limit must be $\Delta \log R$.

Theorem 4.8 If $R$ does not vanish identically, then $R>0$ everywhere. Moreover, Ward's equation (4.5) holds pointwise on $\mathbb{C}$.

Proof Suppose that $R\left(z_{0}\right)=0$. Let $D=D\left(z_{0}, \varepsilon\right)$ be a small disc about $z_{0}$ and consider the measures $\mu=\chi \cdot(1+\Delta \log R)$ and $v=\chi \cdot(1+\Delta \log \tilde{R})$, where $\chi=\mathbf{1}_{D}$. By the previous lemmata, we know that the measures $\mu$ and $\nu$ are both positive, and clearly $\mu=\delta_{z_{0}}+v$. Now consider the Cauchy transform

$$
C^{\mu}(z)=\int_{\mathbb{C}} \frac{1}{z-w} d \mu(w)
$$

Evidently,

$$
C^{\mu}(z)=\frac{1}{z-z_{0}}+C^{\nu}(z), \quad\left|z-z_{0}\right|<\varepsilon,
$$

and $\bar{\partial} C^{v}=v \geq 0$. Now when $\left|z-z_{0}\right|<\varepsilon$, the right-hand side in Ward's equation equals

$$
R(z)-(1+\Delta \log R)(z)=R(z)-\bar{\partial} C^{\mu}(z)
$$

By (4.5), we have

$$
\bar{\partial}\left(C(z)+C^{\mu}(z)\right)=R(z),
$$

and hence (by Weyl's lemma)

$$
C(z)=-\frac{1}{z-z_{0}}-C^{v}(z)+v(z),
$$

where $v$ is smooth in some neighborhood of $z_{0}$. If $C^{\mu}(z)$ remains bounded as $z \rightarrow z_{0}$, then $\mu=v+\delta_{z_{0}}$ cannot contain any point mass at $z_{0}$, so $v$ can be written $-\delta_{z_{0}}+\rho$, where $\rho\left(\left\{z_{0}\right\}\right)=0$. This contradicts the fact that $v \geq 0$. Hence

$$
|C(z)| \rightarrow \infty \quad \text { as } \quad z \rightarrow z_{0}
$$

This contradicts the boundedness of $C$ in Lemma 4.6. Hence $R\left(z_{0}\right)=0$ is impossible. 
We have shown that $R>0$ everywhere. Since Ward's equation $\bar{\partial} C=R-1-$ $\Delta \log R$ holds in the sense of distributions and the right-hand side is smooth, application of Weyl's lemma now shows that $C(z)$ is smooth and that Ward's equation holds pointwise.

\subsection{Reformulation of Ward's Equation}

It is convenient to somewhat reformulate Ward's equation. Given a Hermitian-entire function $\Psi$ (positive on the diagonal in $\mathbb{C}^{2}$ ), we define the functions $R(z)=R^{\Psi}(z)=$ $\Psi(z, z)$ and

$$
D(z)=D^{\Psi}(z)=\int \frac{e^{-|z-w|^{2}}}{z-w}|\Psi(z, w)|^{2} d A(w) .
$$

Thus $D=R C$.

Lemma 4.9 Ward's equation (4.5) is satisfied if and only if there exists a smooth function $P(z)$ such that

$$
\bar{\partial} P=R-1
$$

and

$$
D=P R-\partial R
$$

Proof The equation (4.5) means that

$$
\bar{\partial}(D / R)=R-1-\bar{\partial}(\partial R / R) .
$$

Let $P_{0}$ be an arbitrary solution to the equation $\bar{\partial} P_{0}=R-1$. Then (4.9) becomes

$$
\bar{\partial}\left[\frac{D}{R}-P_{0}+\frac{\partial R}{R}\right]=0 .
$$

This last identity is fulfilled if and only if there is an entire function $E$ such that

$$
D-P_{0} R+\partial R=E R
$$

Setting $P=P_{0}+E$, we see that (4.7) and (4.8) are satisfied. Conversely, if (4.7) and (4.8) hold, then

$$
\bar{\partial}(D / R)=\bar{\partial}(P-\partial R / R)=R-1-\bar{\partial}(\partial R / R) ;
$$

i.e., (4.9) holds.

\subsection{Relations for the Boundary Kernel}

We finish this section by noting the following theorem. 
Theorem 4.10 The kernel $K(z, w)=G(z, w) F(z+\bar{w})$ satisfies Ward's equation and the mass-one equation.

Proof 1 The proof of Ward's equation in Subsection 4.3 and the example of the Ginibre ensemble in Subsection 2.2 show that Ward's equation is satisfied. The mass-one equation can be deduced in a similar way; in fact, we shall prove in Subsection 6 that the mass-one equation is a consequence of Ward's equation in the translation invariant case.

Proof 2 We here give an alternative, direct verification that the function $R(z)=$ $F(2 \operatorname{Re} z)$ satisfies the mass-one equation (3.2). To this end, note that

$$
\lim _{x \rightarrow-\infty} F^{(n)}(x)=\delta_{n 0}, \quad n=0,1,2, \ldots
$$

Using this, we obtain by differentiating in (3.2) the equivalent equation

$$
\sum_{n=0}^{\infty} \frac{F^{(n)}(z) F^{(n+1)}(z)}{n !}=\frac{1}{2} F^{\prime}(z)
$$

Dividing by $F^{\prime}$ and using the Rodrigues formula for the Hermite polynomial $h_{n}$,

$$
h_{n}(z)=(-1)^{n} e^{z^{2} / 2} \frac{d^{n}}{d z^{n}}\left(e^{-z^{2} / 2}\right)=(-1)^{n} \frac{F^{(n+1)}(z)}{F^{\prime}(z)},
$$

one can rewrite $(4.10)$ in the form

$$
F(z)-\frac{1}{2}=F^{\prime}(z) \sum_{n=1}^{\infty} \frac{h_{n-1}(z) h_{n}(z)}{n !}
$$

But both sides of (4.11) have a zero at the origin, so we need only verify that the derivatives are equal. Using the recursion $h_{n}^{\prime}(z)=n h_{n-1}(z)$, one realizes that our assertion is equivalent to that

$$
F^{\prime}(z)=F^{\prime}(z) \sum_{n=1}^{\infty} \frac{1}{n !}\left(n h_{n-1}^{2}(z)+(n-1) h_{n-2}(z) h_{n}(z)-z h_{n-1}(z) h_{n}(z)\right) .
$$

However, since $h_{0}=1$, we have

$$
\sum_{n=1}^{\infty} \frac{1}{n !}\left(n h_{n-1}^{2}-h_{n}^{2}\right)=\sum_{n=1}^{\infty}\left\{\frac{h_{n-1}^{2}}{(n-1) !}-\frac{h_{n}^{2}}{n !}\right\}=1,
$$

so the sum in the right-hand side of (4.12) equals

$$
1+\sum_{n=1}^{\infty} \frac{1}{n !} h_{n}\left(h_{n}+(n-1) h_{n-2}-z h_{n-1}\right)
$$


and this equals 1 by the recursive definition of Hermite polynomials: $h_{-1}=0, h_{0}=1$, and $h_{n}=z h_{n-1}-(n-1) h_{n-2}$ for $n \geq 2$.

\section{A Priori Estimates at Regular Boundary Points}

In this section, we prove the a priori estimates for the one-point function in Theorem 1.4. We will develop the technique of "approximate Bergman projections," which has the advantage of being relatively simple while still giving good enough a priori estimates of the main term in the one-point function $\mathbf{R}_{n}$. (A similar estimate was used earlier in the paper [3].)

We remark that the analysis in this section was adapted to various singular situations in the paper [7], where the reader also can find references to other kinds of related asymptotic expansions.

The section is finished by verifying the $1 / 8$-formula in Theorem 1.5 .

\subsection{Heat Kernel Estimate}

Fix a number $\vartheta<1$ (close to 1 ) and a smooth function $\psi$ with $\psi=1$ in $D(0 ; \vartheta)$ and $\psi=0$ outside $D(0 ; 1)$. For given $\zeta \in \mathbb{C}$ and $\delta>0$, we define $\chi$ by $\chi(\omega)=$ $\psi((\omega-\zeta) / \delta)$. Then $\chi=1$ in $D(\zeta ; \vartheta \delta), \chi=0$ outside $D(\zeta ; \delta)$, and the Dirichlet norm $\|\bar{\partial} \chi\|$ depends only on $\vartheta$. We sometimes write $\chi_{\zeta}$ for $\chi$.

We next fix a sequence $\left(\delta_{n}\right)_{1}^{\infty}$ of positive numbers in the interval $\left(2 \gamma / \sqrt{n}, \delta_{0} / 2\right)$, where $\gamma$ is a sufficiently small positive number independent of $n$, and

$$
n \delta_{n}^{3} \rightarrow 0 \text { as } n \rightarrow \infty
$$

Below we will fix a point $\zeta$ in $S$. We shall use the Hermitian analytic extension $A(\zeta, \eta)$ satisfying $A(\zeta, \zeta)=Q(\zeta)$ for $\zeta \in \Omega$ (a neighborhood of $S$ ). We assume that $\delta_{0}$ is small enough that $A(\zeta, \eta)$ is defined whenever $|\zeta-\eta|<2 \delta_{0}, \zeta \in S$.

We will use the kernels $\mathbf{K}_{n}$ and $\mathbf{K}_{n}^{\#}$, where we recall that (cf. Subsection 3.3)

$$
\mathbf{K}_{n}^{\#}(\zeta, \eta)=n\left(\partial_{1} \bar{\partial}_{2} A\right)(\zeta, \eta) \cdot e^{n A(\zeta, \eta)} e^{-n Q(\zeta) / 2} e^{-n Q(\eta) / 2}
$$

For a fixed $\eta$, we will use abbreviations such as $\mathbf{K}_{\eta}(\zeta)=\mathbf{K}_{n, \eta}(\zeta)=\mathbf{K}_{n}(\zeta, \eta)$, etc. Moreover, if $f$ is a function supported in the domain of the function $\mathbf{K}_{\eta}^{\#}$, we write

$$
\Pi_{n}^{\#} f(\eta)=\left\langle f, \mathbf{K}_{\eta}^{\#}\right\rangle:=\int_{\operatorname{supp} f} f(\zeta) \overline{\mathbf{K}}_{\eta}^{\#}(\zeta) d A(\zeta)
$$

Theorem 5.1 For $\zeta \in \operatorname{Int} S$, define $\delta=\delta(\zeta)=\operatorname{dist}(\zeta, \partial S)$. There is a constant $C$ such that $\delta<\delta_{n}$ implies

$$
\left|\mathbf{K}_{\zeta}(\zeta)-\Pi_{n}\left[\chi_{\zeta} \mathbf{K}_{\zeta}^{\#}\right](\zeta)\right| \leq C M_{n}(\delta) \sqrt{\mathbf{K}_{\zeta}(\zeta)}
$$


where

$$
M_{n}(\delta)=\frac{1}{\sqrt{n \Delta Q(\zeta)}}+\frac{1}{\delta} e^{-n \Delta Q(\zeta)(\vartheta \delta)^{2} / 2}
$$

In particular,

$$
\left|\mathbf{K}_{\zeta}(\zeta)-\Pi_{n}\left[\chi_{\zeta} \mathbf{K}_{\zeta}^{\#}\right](\zeta)\right| \leq C \sqrt{n} M_{n}(\delta) .
$$

The proof relies on the following lemma.

Lemma 5.2 If $f=u e^{-n Q / 2}$, where $u$ is analytic in $D(\zeta ; \delta)$, then

$$
\left|f(\zeta)-\Pi_{n}^{\#}\left[\chi_{\zeta} f\right](\zeta)\right| \leq C M_{n}(\delta)\|f\|
$$

Proof Assume that $\zeta=0$ and write $\chi=\chi_{\zeta}$. Then $\Pi_{n}^{\#}[\chi f](\zeta)$ equals to the integral

$$
I=e^{-n Q(0) / 2} \int \chi(\omega) u(\omega)\left(\partial_{1} \bar{\partial}_{2} A\right)(0, \omega) \cdot n e^{-n(A(\omega, \omega)-A(0, \omega))} d A(\omega)
$$

which means that

$$
I=-e^{-n Q(0) / 2} \int \frac{1}{\omega} u(\omega) \chi(\omega) F(\omega) \bar{\partial}_{\omega}\left[e^{-n(A(\omega, \omega)-A(0, \omega))}\right] d A(\omega),
$$

where

$$
F(\omega)=\frac{\omega\left(\partial_{1} \bar{\partial}_{2} A\right)(0, \omega)}{\bar{\partial}_{2} A(\omega, \omega)-\bar{\partial}_{2} A(0, \omega)}
$$

By Taylor's formula, we have $F(\omega)=1+O(\omega)$ and $\bar{\partial} F(\omega)=O(\omega)$ as $\omega \rightarrow 0$, where the $O$-constant can be chosen independent of $\zeta$.

Integrating by parts in (5.1), one obtains

$$
I=f(0)+e^{-n Q(0) / 2}\left(\epsilon_{1}+\epsilon_{2}\right)
$$

where

$$
\epsilon_{1}=\int \frac{u \cdot \bar{\partial} \chi \cdot F}{\omega} e^{-n[A(\omega, \omega)-A(0, \omega)]}, \quad \epsilon_{2}=\int \frac{u \cdot \chi \cdot \bar{\partial} F}{\omega} e^{-n[A(\omega, \omega)-A(0, \omega)]} .
$$

It follows that there is a constant $C$ (independent of $\zeta, n$, and $\delta$ ) such that

$$
\begin{aligned}
\left|\epsilon_{1}\right| & \leq C \frac{1}{\vartheta \delta} \int|u||\bar{\partial} \chi| e^{-n[Q(\omega)-\operatorname{Re} A(0, \omega)]} \\
\left|\epsilon_{2}\right| & \leq C \int \chi|u| e^{-n[Q(\omega)-\operatorname{Re} A(0, \omega)]}
\end{aligned}
$$


By the remark after Lemma 3.4 and the assumption $n \delta_{n}^{3} \rightarrow 0$, we have the estimate

$$
e^{-n[Q(\omega) / 2-\operatorname{Re} A(0, \omega)]} \leq C e^{n Q(0) / 2-n \Delta Q(0)|\omega|^{2} / 2}, \quad|\omega|<2 \delta_{n} .
$$

Using this and the Cauchy-Schwarz inequality, we now find (since $|\omega| \geq \vartheta \delta$ when $\bar{\partial} \chi(\omega) \neq 0)$

$$
\left|\epsilon_{1}\right| e^{-n Q(0) / 2} \leq C \frac{1}{\delta} e^{-n \Delta Q(0)(\vartheta \delta)^{2} / 2}\|f\|\|\bar{\partial} \chi\| \leq C \frac{1}{\delta} e^{-n \Delta Q(0)(\vartheta \delta)^{2} / 2}\|f\|
$$

and

$$
\left|\epsilon_{2}\right| e^{-n Q(0) / 2} \leq C\|f\|\left(\int_{\mathbb{C}} e^{-n \Delta Q(0)|\zeta|^{2}}\right)^{1 / 2} \leq C \frac{1}{\sqrt{n \Delta Q(0)}}\|f\| .
$$

The proof of the lemma is complete.

Proof of Theorem 5.1 We have that

$$
\overline{\Pi_{n}^{\#}\left[\chi_{\zeta} \mathbf{K}_{\zeta}\right](\zeta)}=\overline{\left\langle\chi_{\zeta} \mathbf{K}_{\zeta}, \mathbf{K}_{\zeta}^{\#}\right\rangle}=\left\langle\chi_{\zeta} \mathbf{K}_{\zeta}^{\#}, \mathbf{K}_{\zeta}\right\rangle=\Pi_{n}\left[\chi_{\zeta} \mathbf{K}_{\zeta}^{\#}\right](\zeta)
$$

whence

$$
\left|\mathbf{K}_{\zeta}(\zeta)-\Pi_{n}\left[\chi_{\zeta} \mathbf{K}_{\zeta}^{\#}\right](\zeta)\right|=\left|\mathbf{K}_{\zeta}(\zeta)-\Pi_{n}^{\#}\left[\chi_{\zeta} \mathbf{K}_{\zeta}\right](\zeta)\right|
$$

It now suffices to take $f=\mathbf{K}_{\zeta}$ in Lemma 5.2 since $\left\|\mathbf{K}_{\zeta}\right\|^{2}=\mathbf{K}_{n}(\zeta, \zeta) \leq C n$ (cf. Lemma 3.3).

\subsection{Bergman Projection Estimate}

Recall that $L_{\phi}^{2}$ denotes the space of functions $f$ normed by $\|f\|_{\phi}^{2}=\int|f|^{2} e^{-\phi}$. We shall let $A_{\phi}^{2}$ denote the subspace of $L_{\phi}^{2}$ consisting of entire functions. We write $\pi_{\phi}$ for the orthogonal (Bergman) projection $L_{\phi}^{2} \rightarrow A_{\phi}^{2}$.

When $\pi$ is the orthogonal projection of a Hilbert space onto a closed subspace, we denote by $\pi^{\perp}=I-\pi$ the complementary projection.

Our starting point is a simple "Hörmander estimate" (cf. [22, p. 250]): if $\phi$ is smooth and strictly subharmonic in $\mathbb{C}$, and if $u \in C_{0}^{\infty}(\mathbb{C})$, then

$$
\left\|\pi_{\phi}^{\perp} u\right\|_{\phi}^{2} \leq \int_{\mathbb{C}}|\bar{\partial} u|^{2} \frac{e^{-\phi}}{\Delta \phi}
$$

Lemma 5.3 Fix $\zeta \in \operatorname{Int} S$. Put $\delta=\operatorname{dist}(\zeta, \partial S)$, and assume that $\delta>2 \gamma / \sqrt{n}$. Then there is a constant $C$ such that

$$
\left|\mathbf{K}_{\zeta}^{\#}(\zeta)-\Pi_{n}\left[\chi_{\zeta} \mathbf{K}_{\zeta}^{\#}\right](\zeta)\right| \leq C n e^{-n \Delta Q(\zeta)(\vartheta \delta)^{2} / 2} .
$$


Proof Let $g_{\zeta}(\omega)=\mathbf{K}_{\zeta}^{\#}(\omega) e^{n Q(\omega) / 2}$. Observe that $g_{\zeta}$ is holomorphic near $\zeta$. Put

$$
u(\omega)=\chi_{\zeta}(\omega) g_{\zeta}(\omega)-\pi_{n}\left[\chi_{\zeta} g_{\zeta}\right](\omega),
$$

and observe that $u$ is a norm-minimal solution in $L_{n Q}^{2}$ to the problem $\bar{\partial} u=\bar{\partial} f$, where $f=\chi_{\zeta} \cdot g_{\zeta}$. We shall prove that

$$
\|u\|_{n Q} \leq C n^{-1 / 2}\left\|\bar{\partial}\left(\chi_{\zeta} \cdot g_{\zeta}\right)\right\|_{n Q} .
$$

To do this, we introduce the strictly subharmonic function

$$
\phi(\omega)=\check{Q}(\omega)+n^{-1} \log \left(1+|\omega|^{2}\right)
$$

and consider $v_{0}=\pi_{n \phi}^{\perp}\left(\chi_{\zeta} g_{\zeta}\right)$. Here $\check{Q}$ is the equilibrium potential, cf. Subsection 3.4.

By the estimate (5.2), we have

$$
\left\|v_{0}\right\|_{n \phi}^{2} \leq \int\left|\bar{\partial}\left(\chi_{\zeta} \cdot g_{\zeta}\right)\right|^{2} \frac{e^{-n \phi}}{n \Delta \phi} d A
$$

Since $\chi_{\zeta}$ is supported in $S$ and since $\Delta \phi>\Delta Q \geq$ const. $>0$ there, we obtain

$$
\left\|v_{0}\right\|_{n \phi} \leq C n^{-1 / 2}\left\|\bar{\partial}\left(\chi_{\zeta} \cdot g_{\zeta}\right)\right\|_{n Q} \cdot
$$

Next note the estimate $n \phi \leq n Q+$ const. on $\mathbb{C}$, which is obvious in view of the growth assumption on $Q$ near infinity. This gives $\left\|v_{0}\right\|_{n Q} \leq C\left\|v_{0}\right\|_{n \phi}$, and we have shown (5.4) with $u=v_{0}$.

Since $n \phi(\omega)=(n+1) \log |\omega|^{2}+O(1)$ as $\omega \rightarrow \infty$ (see Subsection 3.4), we have the equality $A_{n \phi}^{2}=\mathcal{P}_{n}$ in the sense of sets. Hence $u=v_{0}$ solves, in addition to (5.4), the problem

$$
\bar{\partial} u=\bar{\partial}\left(\chi_{\zeta} g_{\zeta}\right) \quad \text { and } \quad u-\chi_{\zeta} g_{\zeta} \in \mathcal{P}_{n} .
$$

Since $g_{\zeta}(\omega)=\mathbf{k}_{n}^{\#}(\omega, \zeta) e^{-n Q(\zeta) / 2}$ is analytic, we have $\bar{\partial} u=\bar{\partial} \chi_{\zeta} \cdot g_{\zeta}$. Recalling that $\mathbf{k}_{n}^{\#}=n\left(\partial_{1} \bar{\partial}_{2} A\right) e^{n A}$, the remark after Lemma 3.4 gives

$$
|\bar{\partial} u(\omega)|^{2} e^{-n Q(\omega)} \leq C n^{2}\left|\bar{\partial} \chi_{\zeta}(\omega)\right|^{2} e^{-n \Delta Q(\zeta)|\omega-\zeta|^{2}} .
$$

Since $|\omega-\zeta| \geq \vartheta \delta$ when $\bar{\partial} \chi_{\zeta}(\omega) \neq 0$, we find

$$
\left|\bar{\partial}\left(\chi_{\zeta} g_{\zeta}\right)(\omega)\right|^{2} e^{-n Q(\omega)} \leq C n^{2}\left|\bar{\partial} \chi_{\zeta}(\omega)\right|^{2} e^{-n \Delta Q(\zeta)(\vartheta \delta)^{2}} .
$$

We have shown that

$$
\left\|\bar{\partial}\left(\chi_{\zeta} g_{\zeta}\right)\right\|_{n Q} \leq C n e^{-n \Delta Q(\zeta)(\vartheta \delta)^{2} / 2} .
$$


Applying the estimate (5.4), one obtains

$$
\|u\|_{n Q} \leq C \sqrt{n} e^{-n \Delta Q(\zeta)(\vartheta \delta)^{2} / 2} .
$$

We shall now finally use the assumption that $\delta \geq \gamma / \sqrt{n}$. This gives that the function $u$ is analytic in the disc $D(\zeta ; \gamma / \sqrt{n})$, so that Lemma 3.1 applies. We obtain that

$$
|u(\zeta)|^{2} e^{-n Q(\zeta)} \leq C n\|u\|_{n Q}^{2},
$$

where $C$ depends on $\gamma$ and $\Delta Q(\zeta)$. Combining with (5.5), we have shown (with a new $C$ )

$$
\left|g_{\zeta}(\zeta)-\pi_{n}\left[\chi_{\zeta} g_{\zeta}\right](\zeta)\right| e^{-n Q(\zeta) / 2} \leq C n e^{-n \Delta Q(\zeta)(\vartheta \delta)^{2} / 2}
$$

The proof of the lemma is complete.

The following result is just a restatement of part (ii) of Theorem 1.4.

Theorem 5.4 Fix a constant $\ell<1 / 2$. There is then a constant $C$ such that if $\zeta \in S$ and $\delta=\operatorname{dist}(\zeta, \partial S)$, then

$$
\left|\mathbf{K}_{n}(\zeta, \zeta)-\mathbf{K}_{n}^{\#}(\zeta, \zeta)\right| \leq C\left(1+n e^{-n \Delta Q(\zeta) \ell \delta^{2}}\right)
$$

Proof By Lemma 3.3, we have $\left\|\mathbf{K}_{\zeta}\right\|^{2} \leq C n$. This gives that (5.6) holds trivially when $\delta<\gamma / \sqrt{n}$ (because $\mathbf{K}_{n}(\zeta, \zeta) \leq C n$ and $\mathbf{K}_{n}^{\#}(\zeta, \zeta) \leq C n$ for sufficiently large $C)$. We can thus assume that $\delta>\gamma / \sqrt{n}$. To this end, we put $\ell=\vartheta^{2} / 2$. Then

$$
\begin{aligned}
\left|\mathbf{K}_{\zeta}(\zeta)-\mathbf{K}_{\zeta}^{\#}(\zeta)\right| & \leq\left|\mathbf{K}_{\zeta}(\zeta)-\Pi_{n}\left[\chi_{\zeta} \mathbf{K}_{\zeta}^{\#}\right](\zeta)\right|+\left|\Pi_{n}^{\perp}\left[\chi_{\zeta} \mathbf{K}_{\zeta}^{\#}\right](\zeta)\right| \\
& \leq C\left(1+n e^{-n \Delta Q(\zeta) \ell \delta^{2}}\right)+C n e^{-n \Delta Q(\zeta) \ell \delta^{2}},
\end{aligned}
$$

where we have used Theorem 5.1 to estimate the first term and (5.3) for the second one.

\subsection{An Exterior Estimate}

Recall from Lemma 3.3 that there is a constant $C$ such that

$$
\mathbf{R}_{n}(\zeta) \leq C n e^{-n(Q-\check{Q})(\zeta)}, \quad \zeta \in \mathbb{C}
$$

Now fix a regular boundary point and rescale in the usual way:

$$
z=e^{-i \theta} \varrho_{n}(\zeta-p), \quad R_{n}(z)=\varrho_{n}^{-2} \mathbf{R}_{n}(\zeta), \quad\left(\varrho_{n}=\sqrt{n \Delta Q(p)}\right) .
$$

Let $N=e^{i \theta}$ be the outer normal direction at $p$. 
Lemma 5.5 Suppose that $p$ is a regular boundary point at distance at least $\delta$ from all singular boundary points, where $\delta>0$ is independent of $n$. There is then a constant $C=C(\delta)$ such that whenever $\zeta \in S^{c}$ and $|z| \leq \log n$, we have

$$
R_{n}(z) \leq C e^{-2 x^{2}}, \quad z=x+i y .
$$

Proof Let $V$ be the harmonic continuation of $\left.\check{Q}\right|_{S^{c}}$ to a neighborhood of $p$. (We can choose the coordinate system so that $\theta=0$ and the exterior normal derivative " $\partial / \partial n$ " (having nothing to do with the integer $n$ ) is simply $\partial / \partial x$.)

By Taylor's formula, we have, for $x>0$, with $M=Q-V$,

$$
M\left(p+x N / \varrho_{n}\right)=\frac{1}{2 \varrho_{n}^{2}} \frac{\partial^{2} M}{\partial x^{2}}(p) x^{2}+\frac{1}{6 \varrho_{n}^{3}} \frac{\partial^{3} M}{\partial x^{3}}\left(p+x_{*} N / \varrho_{n}\right) x^{3},
$$

where $x_{*}=x_{*}(n, p, x)$ is between 0 and $x$. However, since $p$ is a regular point and $Q=V$ on $\partial S$, we have $\partial^{2} M / \partial s^{2}(p)=0$, where $\partial / \partial s$ denotes differentiation in the tangential direction. Adding this to the above Taylor expansion, using that $\left(\partial^{2} / \partial s^{2}+\partial^{2} / \partial n^{2}\right) M=4 \Delta M=4 \Delta Q$, we obtain, when $|z| \leq C \log n$,

$$
n M\left(p+z N / \varrho_{n}\right)=2 x^{2}+O\left(\log ^{3} n / \sqrt{n}\right), \quad 0 \leq x \leq C \log n
$$

The proof of the lemma is complete.

It follows from the lemma that each limiting 1-point function $R(z)=K(z, z)$ at a regular boundary point must satisfy $R(z) \leq C e^{-2 x^{2}}$, where $x=\operatorname{Re} z$. This proves Theorem 1.4, part (i).

\subsection{The 1/8-Formula}

We now prove Theorem 1.5. Suppose that the droplet $S$ is connected and that the boundary $\partial S$ is everywhere regular, so that the theory from [3] applies. Consider the class $\mathcal{C}_{0}$ of test functions $f \in C_{0}^{\infty}(\mathbb{C})$ with $f=0$ on $\partial S$. For $f \in \mathcal{C}_{0}$, we define functionals

$$
\rho_{n}(f)=\int_{\mathbb{C}} f \cdot\left(\mathbf{R}_{n}-\left(n \Delta Q+\frac{1}{2} \Delta \log \Delta Q\right) \cdot \mathbf{1}_{S}\right) d A .
$$

We shall use the main result of the paper [3], which implies that the limit $\rho(f)=$ $\lim \rho_{n}(f)$ exists and equals

$$
\rho(f)=\frac{1}{8 \pi} \int_{\partial S} \frac{\partial f}{\partial n} d s,
$$

where $\partial f / \partial n$ is the exterior normal derivative and $d s$ is arclength measure on $\partial S$. 
Next fix a parameter $M>0$, and consider the tubular $M / \sqrt{n \Delta Q}$-neighborhood of $\partial S$, defined by

$$
\mathcal{N}_{M}:=\{p+\eta ; p \in \partial S,|\eta|<M / \sqrt{n \Delta Q(p)}\}
$$

To simplify, we assume that $\partial S$ is connected; a simple modification will prove the general case.

Now consider an arclength parametrization $p=p(s)\left(0 \leq s \leq s_{0}\right)$ of $\partial S$. Also denote by $N_{p}$ the exterior unit normal at $p \in \partial S$.

Define a coordinate system $(s, t)$ where $s$ and $t$ are real parameters, related to the corresponding point $\zeta=\zeta_{n}(s, t) \in \mathcal{N}_{M}$ by

$$
\zeta=p(s)+\frac{t}{\sqrt{n \Delta Q(p(s))}} N_{p(s)}
$$

In the $(s, t)$-system, the set $\mathcal{N}_{M}$ corresponds to a strip

$$
\left\{(s, t) ; 0 \leq s \leq s_{0},-M<t<M\right\}
$$

A simple geometric consideration shows that the area element satisfies the relation

$$
d A(\zeta)=(1+o(1)) \frac{1}{\pi} \frac{1}{\sqrt{n \Delta Q(p(s))}} d s d t, \quad\left(\zeta=\zeta_{n}(s, t) \in \mathcal{N}_{M}\right)
$$

Here $o(1) \rightarrow 0$ as $n \rightarrow \infty$, and the $o(1)$-constant depends on $M$.

The rescaled 1-point function about $p(s)$ will be denoted by

$$
R_{n, p(s)}(t):=\frac{1}{n \Delta Q(p(s))} \mathbf{R}_{n}\left(p(s)+\frac{t}{\sqrt{n \Delta Q(p(s))}} N_{p(s)}\right), \quad(t \in \mathbb{R})
$$

We now define the functionals

$$
\begin{aligned}
& \rho_{n}^{\prime}(f)=\int_{\mathcal{N}_{M}} f \cdot\left(\mathbf{R}_{n}-\left(n \Delta Q+\frac{1}{2} \Delta \log \Delta Q\right) \cdot \mathbf{1}_{S}\right) d A, \\
& \rho_{n}^{\prime \prime}(f)=\int_{\mathbb{C} \backslash \mathcal{N}_{M}} f \cdot\left(\mathbf{R}_{n}-\left(n \Delta Q+\frac{1}{2} \Delta \log \Delta Q\right) \cdot \mathbf{1}_{S}\right) d A .
\end{aligned}
$$

Clearly, $\rho_{n}(f)=\rho_{n}^{\prime}(f)+\rho_{n}^{\prime \prime}(f)$.

To study $\rho_{n}^{\prime}(f)$, we use Taylor's formula in the tubular neighborhood $\mathcal{N}_{M}$,

$$
f\left(\zeta_{n}(s, t)\right)=\frac{\partial f}{\partial n}(p(s)) \cdot \frac{t}{\sqrt{n \Delta Q(p(s))}}+O(1 / n)
$$


This gives

$\rho_{n}^{\prime}(f)=(1+o(1)) \frac{1}{\pi} \int_{\partial S} \frac{\partial f}{\partial n}(p(s)) d s \int_{-M}^{M} t \cdot\left(R_{n, p(s)}(t)-\mathbf{1}(t)\right) d t, \quad\left(\chi=\chi_{(-\infty, 0)}\right)$.

We have here made a change of variables in the integral defining $\rho_{n}^{\prime}(f)$ and used relation (5.8).

Next, the a priori estimates in Theorem 1.4 imply that there are constants $C, c>0$ such that

$$
\left|\mathbf{R}_{n}(\zeta)-n \Delta Q(\zeta) \mathbf{1}_{S}(\zeta)\right| \leq C n e^{-c n \delta(\zeta)^{2}}, \quad \delta(\zeta):=\operatorname{dist}(\zeta, \partial S)
$$

Now for $f \in \mathcal{C}_{0}$, let us write $\tilde{f}(\zeta)=f(\zeta) / \delta(\zeta)$, so $\tilde{f} \in C_{0}^{\infty}(\mathbb{C})$.

The estimates in (5.9) give that there are constants $C, C^{\prime}$ such that

$$
\left|\rho_{n}^{\prime \prime}(f)\right| \leq C\|\tilde{f}\|_{\infty} \int_{M}^{\infty} t e^{-c t^{2}} d t+C^{\prime} \int_{S}|f| d A .
$$

By (5.7) we now get

$$
\begin{aligned}
& (1+o(1)) \frac{1}{\pi}\left|\int_{\partial S} \frac{\partial f}{\partial n}(p(s)) d s\left[\int_{-M}^{M} t \cdot\left(R_{n, p(s)}(t)-\mathbf{1}(t)\right) d t-\frac{1}{8}\right]\right|=\left|\rho_{n}^{\prime \prime}(f)\right| \\
& \quad \leq C\|\tilde{f}\|_{\infty} e^{-c M^{2}}+C^{\prime}\|f\|_{1} .
\end{aligned}
$$

It is convenient to introduce a notation for the expression appearing in the inner integral,

$$
h_{n, M}(s):=\int_{-M}^{M} t \cdot\left(R_{n, p(s)}(t)-\mathbf{1}(t)\right) d t-\frac{1}{8} .
$$

Lemma 5.6 For almost all $s \in\left[0, s_{0}\right]$, we have

$$
\lim _{M \rightarrow \infty} \lim _{n \rightarrow \infty} h_{n, M}(s)=0 .
$$

Proof We define auxiliary functions

$$
h^{*}(s)=\limsup _{M \rightarrow \infty} \limsup _{n \rightarrow \infty} h_{n, M}(s), \quad h_{*}(s)=\liminf _{M \rightarrow \infty} \liminf _{n \rightarrow \infty} h_{n, M}(s) .
$$

We must prove that $h^{*}(s) \leq 0$ and $h_{*}(s) \geq 0$ for almost every $s$. The two cases are similar, so we just prove the inequality for $h^{*}$.

Suppose that the set $E_{\alpha}:=\left\{h^{*}>\alpha\right\}$ has positive measure for some $\alpha>0$. Take $\varepsilon \in(0,1)$ to be fixed later. 
Since the set $E_{\alpha}$ has positive measure, it contains a point of density (see, e.g., [35]); i.e., there is $x \in E_{\alpha}$ and $\delta>0$ such that

$$
\left|E_{\alpha} \cap(x-u, x+u)\right| \geq(1-\varepsilon) \cdot 2 u, \quad(0<u<\delta) .
$$

(Here $|E|$ denotes Lebesgue measure of a set $E \subset \mathbb{R}$.)

Put $\omega=(x-\delta, x+\delta)$ and $\tilde{\omega}=(x-\delta / 2, x+\delta / 2)$. Also pick a large number $P>0$.

Now fix a test function $f \in \mathcal{C}_{0}$ such that $0 \leq \partial f / \partial n \leq P$ on $\partial S, \partial f / \partial n=0$ outside $\omega, \partial f / \partial n=P$ on $\tilde{\omega}$, and $\|f\|_{1} \leq 1$.

Taking $\lim \sup _{n \rightarrow \infty}$ and then $\lim _{\sup _{M \rightarrow \infty}}$ in (5.10), we find that

$$
\int_{\omega \cap E_{\alpha}} \frac{\partial f}{\partial n}(p(s)) h^{*}(s) d s+\int_{\omega \backslash E_{\alpha}} \frac{\partial f}{\partial n}(p(s)) h^{*}(s) d s \leq C^{\prime \prime} .
$$

It is easy to see that $h^{*}$ is bounded from below, say $h^{*} \geq-m$, so by (5.11),

$$
-\int_{\omega \backslash E_{\alpha}} \frac{\partial f}{\partial n}(p(s)) h^{*}(s) d s \leq m P \varepsilon \cdot 2 \delta,
$$

and hence

$$
\int_{\omega \cap E_{\alpha}} \frac{\partial f}{\partial n}(p(s)) h^{*}(s) d s \leq 2 m \varepsilon \delta P+C^{\prime \prime} .
$$

Since $\partial f / \partial n=P$ on $\tilde{\omega}$ while $h^{*} \geq \alpha$ on $E_{\alpha}$, the integral in the left-hand side is at least $\left|E_{\alpha} \cap \tilde{\omega}\right| \cdot P \alpha \geq(1-\varepsilon) \delta P \alpha$. We have shown that

$$
(1-\varepsilon) \delta \alpha P \leq P \cdot m \varepsilon \cdot 2 \delta+C^{\prime \prime}
$$

Choosing $\varepsilon>0$ small enough and $P$ large enough, we can get a contradiction regardless of the values of $\alpha>0, m$, and $C^{\prime \prime}$. The contradiction shows that $\left|E_{\alpha}\right|=0$.

The conclusion of Lemma 5.6 means that there exists a set $N \subset \partial S$ of arclength zero such that if $p \in(\partial S) \backslash N$, then

$$
\lim _{n \rightarrow \infty} \int_{-\infty}^{+\infty} t \cdot\left(R_{n, p}(t)-\mathbf{1}(t)\right) d t=\frac{1}{8}
$$

Now fix any $p \in(\partial S) \backslash N$. Use compactness to choose a subsequence $n_{k}$ such that $R_{n_{k}, p}$ converges locally uniformly to some limit $R_{p}$. We then have 


$$
\begin{aligned}
\lim _{n \rightarrow \infty} & \int_{-\infty}^{+\infty} t \cdot\left(R_{n, p}(t)-\mathbf{1}(t)\right) d t=\lim _{k \rightarrow \infty} \int_{-\infty}^{+\infty} t \cdot\left(R_{n_{k}, p}(t)-\mathbf{1}(t)\right) d t \\
& =\int_{-\infty}^{+\infty} t \cdot \lim _{k \rightarrow \infty}\left(R_{n_{k}, p}(t)-\mathbf{1}(t)\right) d t=\int_{-\infty}^{+\infty} t \cdot\left(R_{p}(t)-\mathbf{1}(t)\right) d t .
\end{aligned}
$$

We have by this completely proved Theorem 1.5 .

\section{Translation Invariant Solutions}

In this section, we study t.i. (translation invariant) limiting kernels and prove Theorems 1.6-1.9. The point of the translation invariance hypothesis is that we can interpret Ward's equation as a convolution equation, which can be solved using Fourier analysis. If we rescale at a regular boundary point, the solution is fixed uniquely by this and our previously obtained a priori conditions.

\subsection{The Convolution Representation of a Translation Invariant Limiting Kernel}

Let $\gamma(z)=\frac{1}{\sqrt{2 \pi}} e^{-z^{2} / 2}$. In this section, we prove the following result:

Lemma 6.1 Let $K(z, w)=G(z, w) \Phi(z+\bar{w})$ be an arbitrary translation invariant limiting kernel. Then there exists a Borel function $f$ with $0 \leq f \leq 1$ such that $\Phi=\gamma * f$.

Recall that a limiting kernel $K=G \Psi$ is called translation invariant (or t.i.) if

$$
\Psi(z+i t, w+i t)=\Psi(z, w), \quad t \in \mathbb{R} .
$$

Let us start the discussion of this important case by proving a simple lemma.

Lemma 6.2 $\Psi$ is translation invariant if and only if $\Psi(z, w)=\Phi(z+\bar{w})$ for some entire function $\Phi$.

Proof If $\Psi$ is $t . i$., we define $\Phi(z)=\Psi(z, 0)$. We must prove that

$$
\Psi(z, w)=\Psi(z+\bar{w}, 0) .
$$

However, for fixed $z$, both functions are analytic in $\bar{w}$, and they coincide on the imaginary axis.

In the following, we fix any limiting holomorphic kernel $L(z, w)=e^{z \bar{w}} \Psi(z, w)$. We shall apply Theorem 1.2 part (iii), which states that both $L$ and $\tilde{L}(z, w)=e^{z \bar{w}}(1-$ $\Psi(z, w))$ are positive kernels.

In the following, we will use the convolution operation

$$
\gamma * \mu(z)=\int_{\mathbb{R}} \gamma(z-t) d \mu(t),
$$


where $\mu$ is a positive measure on $\mathbb{R}$. If $d \mu(t)=f(t) d t$ is absolutely continuous, we write, as before, $\gamma * \mu=\gamma * f$.

We now use the positivity of $L$ only on the imaginary axis, to conclude that for all finite subsets $\left\{x_{i}\right\}_{1}^{N} \subset \mathbb{R}$ and all complex scalars $\alpha_{i}$, we have

$$
\sum \alpha_{j} \bar{\alpha}_{k} e^{x_{j} x_{k}} \Phi\left(i x_{j}-i x_{k}\right) \geq 0
$$

Make the substitution $\Phi(i z)=V(z) e^{z^{2} / 2}$. The positivity condition above then becomes

$$
\sum \alpha_{j} \bar{\alpha}_{k} e^{x_{j}^{2} / 2+x_{k}^{2} / 2} V\left(x_{j}-x_{k}\right) \geq 0, \quad\left(x_{j}\right)_{1}^{N} \subset \mathbb{R}
$$

This can be written

$$
\sum \beta_{j} \bar{\beta}_{k} V\left(x_{j}-x_{k}\right) \geq 0, \quad\left(\beta_{j}=\alpha_{j} e^{x_{j}^{2} / 2}\right)
$$

The function $V(x)$ is hence positive definite, and by Bochner's theorem (e.g., [24]), it is the inverse Fourier transform of a positive measure $\mu$. We have shown that

$$
\Phi(i x) e^{(i x)^{2} / 2}=\int_{\mathbb{R}} e^{i t x} d \mu(t), \quad x \in \mathbb{R} .
$$

Since the entire function $\Phi$ is determined by its values on the imaginary axis, this gives

$$
\Phi(z)=\int_{\mathbb{R}} e^{t z-z^{2} / 2} d \mu(t)=\int_{\mathbb{R}} e^{-(t-z)^{2} / 2} e^{t^{2} / 2} d \mu(t), \quad z \in \mathbb{C} .
$$

Writing $d \nu(t)=\sqrt{2 \pi} e^{t^{2} / 2} d \mu(t)$, we now have the representation

$$
\Phi=\gamma * v
$$

where $v$ is some positive measure.

Similarly, since the kernel $\tilde{L}(z, w)=e^{z \bar{w}}(1-\Phi(z+\bar{w}))$ is a positive kernel (see Theorem 1.3, cf. Subsection 3.7), there is a positive measure $v_{1}$ so that

$$
1-\Phi=\gamma * v_{1}
$$

The positive measures $v$ and $v_{1}$ have the property that

$$
\gamma *\left(v+v_{1}\right)=\Phi+(1-\Phi)=1=\gamma * \mathbf{1}_{\mathbb{R}}
$$

The map $\rho \mapsto \gamma * \rho$ is 1-1 (as can be seen by taking Fourier transforms), so we obtain

$$
v+v_{1}=\mathbf{1}_{\mathbb{R}} .
$$


As both $v$ and $v_{1}$ are positive, this forces both measures to be absolutely continuous, $d v(x)=f(x) d x$ and $d v_{1}(x)=f_{1}(x) d x$, where $f$ and $f_{1}$ are some non-negative Borel functions with $f(x)+f_{1}(x)=1$. In particular, $0 \leq f \leq 1$. By this, the proof of Lemma 6.1 is complete.

\subsection{Translation Invariant Solutions to Ward's Equation}

We shall now prove Theorem 1.6. Thus we shall find all solutions to Ward's equation of the special form $K(z, w)=G(z, w) \Phi(z+\bar{w})$, where $\Phi=\gamma * f$ for some bounded Borel function $f$.

Thus assume that $\Phi=\gamma * f$ is an entire function. It will be convenient to denote the restriction to $\mathbb{R}$ by $J:=\left.\Phi\right|_{\mathbb{R}}$; i.e.,

$$
J(x)=R(x / 2), \quad x \in \mathbb{R} .
$$

Observe that a function $V(z)$, which is translation invariant in the sense that $V(z+i t)=$ $V(z)$ for all $z \in \mathbb{C}$ and $t \in \mathbb{R}$, satisfies $\partial V=\frac{1}{2} \partial_{x} V$. It is convenient to formulate the following reformulation of Ward's equation in terms of $J$ :

Lemma 6.3 A translation invariant kernel $\Psi(z, w)=\Phi(z+\bar{w})$ satisfies Ward's equation if and only if there exists a smooth function $G$ on $\mathbb{R}$, such that

$$
G^{\prime}=J-1
$$

and

$$
L=G J-J^{\prime}
$$

where

$$
L(x)=\int_{\mathbb{C}} \frac{e^{-|w|^{2}}}{w}|\Phi(x-w)|^{2} d A(w), \quad x \in \mathbb{R} .
$$

Proof Set $G(x)=P(x / 2)$ and $L(x)=D(x / 2)$ in Lemma 4.9, where we recall that $D(z)$ is defined by the integral (4.6).

We will need two elementary lemmas.

Lemma 6.4 For all $s, t \in \mathbb{C}$, we have

$$
\int_{\mathbb{C}} e^{-|w|^{2}} e^{i w t} e^{i \bar{w} s} d A(w)=e^{-s t}
$$

Proof A Taylor expansion of $r \mapsto e^{i r\left(t e^{i \theta}+s e^{-i \theta}\right)}$ around $r=0$ gives

$$
\int_{0}^{\infty} e^{-r^{2}} e^{i r\left(t e^{i \theta}+s e^{-i \theta}\right)} r d r=\frac{1}{2} \sum_{n=0}^{\infty} \frac{i^{n} \Gamma(1+n / 2)}{n !}\left(t e^{i \theta}+s e^{-i \theta}\right)^{n} .
$$


If $n$ is odd, the zeroth Fourier coefficient of $\left(t e^{i \theta}+s e^{-i \theta}\right)^{n}$ vanishes, while if $n$ is even, then

$$
\frac{1}{2 \pi} \int_{0}^{2 \pi}\left(t e^{i \theta}+s e^{-i \theta}\right)^{n} d \theta=(s t)^{n / 2}\left(\begin{array}{c}
n \\
n / 2
\end{array}\right)
$$

We have shown that

$$
\frac{1}{\pi} \int_{0}^{2 \pi} d \theta \int_{0}^{\infty} e^{-r^{2}} e^{i r\left(t e^{i \theta}+s e^{-i \theta}\right)} r d r=\sum_{k=0}^{\infty} \frac{(-s t)^{k}}{k !}=e^{-s t}
$$

finishing the proof of the lemma.

Lemma 6.5 For all $s, t \in \mathbb{C}$, we have

$$
\int_{\mathbb{C}} \frac{e^{-|w|^{2}}}{w} e^{i t w} e^{i s \bar{w}} d A(w)=i \frac{1-e^{-s t}}{s}
$$

Proof Fix $s$, and write $I(t)$ for the left-hand side in (6.3). Then $I(0)=0$, and Lemma 6.4 shows that $I^{\prime}(t)=i e^{-s t}$. It follows that

$$
I(t)=i \int_{0}^{t} e^{-s \tau} d \tau=i \frac{1-e^{-s t}}{s}
$$

The proof of the lemma is complete.

Since we are assuming that $\Phi=\gamma * f$ for some suitable function $f$, the restriction $J$ of $\Phi$ to $\mathbb{R}$ has the structure of the usual convolution

$$
J=\gamma * f
$$

We will use the Fourier transform of the function $J$ in a suitable generalized sense:

$$
\hat{J}:=\hat{\gamma} \cdot \hat{f}
$$

where $f$ is regarded as a tempered distribution. This is well defined, since $\hat{\gamma}=\sqrt{2 \pi} \gamma$ is a Schwartz test function.

We will frequently use the following consequence of Fourier's inversion theorem:

$$
\Phi(z)=\frac{1}{2 \pi} \int_{-\infty}^{+\infty} e^{i z t} \hat{J}(t) d t
$$

Here the integral is interpreted as the value of the distribution $\hat{f}$ applied to the Schwartz test function $t \mapsto \hat{\gamma}(t) e^{i z t}$. 
With these conventions, we conclude that

$$
\Phi(x-w)=\frac{1}{2 \pi} \int_{\mathbb{R}} e^{i(x-w) t} \hat{J}(t) d t, \quad \Phi(x-\bar{w})=\frac{1}{2 \pi} \int_{\mathbb{R}} e^{i(x-\bar{w}) s} \hat{J}(s) d s .
$$

Multiplying these identities together, we find that

$$
|\Phi(x-w)|^{2}=\frac{1}{(2 \pi)^{2}} \iint_{\mathbb{R}^{2}} e^{i x(t+s)} e^{-i w t} e^{-i \bar{w} s} \hat{J}(t) \hat{J}(s) d s d t
$$

Now recall the expression for the function $L(x)$ in (6.2). Using (6.5) and Lemma 6.5, we have

$$
\begin{aligned}
L(x) & =\frac{1}{(2 \pi)^{2}} \iint_{\mathbb{R}^{2}} e^{i x(s+t)} \hat{J}(t) \hat{J}(s) d s d t \int_{\mathbb{C}} \frac{e^{-|w|^{2}}}{w} e^{-i w t-i \bar{w} s} d A(w) \\
& =\frac{i}{(2 \pi)^{2}} \iint_{\mathbb{R}^{2}} \frac{e^{-s t}-1}{s} e^{i x(s+t)} \hat{J}(t) \hat{J}(s) d s d t .
\end{aligned}
$$

Next note that the relation $J=G^{\prime}+1$ (see (6.1)) means that

$$
\hat{J}(s)=i s \hat{G}(s)+2 \pi \delta(s),
$$

where $\delta$ is the Dirac measure at 0 . Inserting this in the last expression for $L(x)$, we get

$$
\begin{aligned}
L(x)= & \frac{1}{(2 \pi)^{2}} \iint_{\mathbb{R}^{2}}\left(1-e^{-s t}\right) e^{i x(t+s)} \hat{J}(t) \hat{G}(s) d s d t \\
& +\frac{i}{2 \pi} \int_{\mathbb{R}} \lim _{s \rightarrow 0} \frac{e^{-s t}-1}{s} \cdot e^{i x t} \hat{J}(t) d t \\
= & G(x) J(x)-\frac{1}{(2 \pi)^{2}} \iint_{\mathbb{R}^{2}} e^{-s t} e^{i x(s+t)} \hat{J}(t) \hat{G}(s) d s d t-J^{\prime}(x),
\end{aligned}
$$

where we have used that

$$
\frac{1}{(2 \pi)^{2}} \iint_{\mathbb{R}^{2}} e^{i x(s+t)} \hat{J}(t) \hat{G}(s) d s d t=J(x) G(x)
$$

and also that

$$
-\frac{i}{2 \pi} \int_{\mathbb{R}} t e^{i x t} \hat{J}(t) d t=-\frac{d}{d x} \frac{1}{2 \pi} \int_{\mathbb{R}} e^{i x t} \hat{J}(t) d t=-J^{\prime}(x) .
$$

In view of Lemma 6.3, Ward's equation is equivalent to that $L=G J-J^{\prime}$. Comparing with the last expression for $L(x)$, we have arrived at the following result: 
Lemma 6.6 Under the conditions above, Ward's equation is satisfied if and only if we have $J=G^{\prime}+1$ with a function $G$ such that

$$
\iint_{\mathbb{R}^{2}} e^{-s t} e^{i x(s+t)} \hat{J}(t) \hat{G}(s) d s d t=0 .
$$

We now prove Theorem 1.6.

Recall that $J=\left.\Phi\right|_{\mathbb{R}}$ and $\Phi=\gamma * f$. Let $g$ be a continuous function on $\mathbb{R}$ such that $g^{\prime}=f-1$; this determines $g$ up to a constant. Let us define $G=g * \gamma$. Then

$$
G^{\prime}=g^{\prime} * \gamma=(f-1) * \gamma=J-1 .
$$

By Lemma 6.6, Ward's equation is equivalent to the identity (6.6) for a suitable choice of integration constant for $g$. We can rewrite (6.6) in the form

$$
\begin{aligned}
0 & =\iint_{\mathbb{R}^{2}} e^{-(s+t)^{2} / 2} e^{i x(s+t)} \hat{f}(t) \hat{g}(s) d s d t \\
& =\int_{\mathbb{R}} d \xi e^{-\xi^{2} / 2} e^{i x \xi} \int_{\mathbb{R}} \hat{g}(\xi-t) \hat{f}(t) d t=\int_{\mathbb{R}} e^{i x \xi} e^{-\xi^{2} / 2}(\hat{g} * \hat{f})(\xi) d \xi \\
& =\mathcal{F}^{-1}[\hat{\gamma} \cdot(\hat{g} * \hat{f})](x)=2 \pi(g f) * \gamma(x) .
\end{aligned}
$$

This means that $g f=0$ in the sense of distributions and hence as measurable functions. Let

$$
E=\{x \in \mathbb{R} ; g(x)=0\} .
$$

Then $E$ is a closed set, and the complement $E^{c}=\mathbb{R} \backslash E$ can be written as a countable union of disjoint open intervals $I_{j}$. On each $I_{j}$, we have $f=0$ and $g^{\prime}=-1$ almost everywhere. Since $g=0$ at the endpoints, none of the intervals can be finite. Hence $E$ is connected. Differentiating the relation $f g=0$ and using $g^{\prime}=f-1$, we obtain that $f=f^{2}$ when $f \neq 0$. Hence $f=\mathbf{1}_{E}$ almost everywhere. We have shown that $\Phi$ is representable in the form

$$
\Phi(z)=\gamma * \mathbf{1}_{E}(z)=\frac{1}{\sqrt{2 \pi}} \int_{E} e^{-(z-t)^{2} / 2} d t .
$$

The proof of Theorem 1.6 is finished.

\subsection{Translation Invariant Limiting Kernels at Regular Boundary Points}

In this subsection, we prove Theorem 1.7.

Theorem 6.7 If the limiting kernel $K$ at a regular boundary point is translation invariant, then $K(z, w)=G(z, w) \Phi(z+\bar{w})$, and there is some a such that $\Phi=\gamma * \mathbf{1}_{(-\infty, a)}$. Furthermore, if $R(z)=\Phi(z+\bar{z})$ satisfies $\int_{\mathbb{R}} t \cdot(R(t)-\mathbf{1}(t)) d t=\frac{1}{8}$, then $\Phi=F$ is the plasma function. 
Proof By Lemmata 6.1 and 6.2, $\Psi(z, w)=\Phi(z+\bar{w})$, where $\Phi=\gamma * f$ for some bounded function $f$ with $0 \leq f \leq 1$. By Theorem 1.4, we know that $R(x) \rightarrow 1$ as $x \rightarrow-\infty$ and $R(x) \rightarrow 0$ as $x \rightarrow+\infty$. Moreover, by Theorem 1.6, we can write $\Phi=\gamma * \mathbf{1}_{(-\infty, a)}$ for some $a \in \mathbb{R}$. For the last statement, we must prove that $a=0$.

Let us first prove that

$$
\int_{-\infty}^{\infty} t\left(F(2 t)-\chi_{(-\infty, 0)}(t)\right) d t=\frac{1}{8}
$$

where $F=\gamma * \mathbf{1}$ is the usual plasma function. To this end, recall that $F(t)=\mathbb{P}(X \geq t)$, where $X$ is a standard normal random variable. Hence the integral in the right-hand side can be written

$$
\begin{aligned}
& \int_{-\infty}^{0} t(\mathbb{P}(X \geq 2 t)-1) d t+\int_{0}^{\infty} t \mathbb{P}(X \geq 2 t) d t=2 \int_{0}^{\infty} t \mathbb{P}(X \geq 2 t) d t \\
& =\frac{1}{8} \mathbb{E} X^{2}=\frac{1}{8}
\end{aligned}
$$

We now return to the $t$.i. limiting kernel $R$. We know that $\int_{\mathbb{R}} t(R(t)-\mathbf{1}(t)) d t=\frac{1}{8}$. As we observed above, we can also write $R(x)=\gamma * \mathbf{1}_{(-\infty, a)}(2 x)=F(2 x-a)$ for some $a \in \mathbb{R}$, and we must prove that $a=0$. However, by (6.7),

$$
0=\int_{\mathbb{R}} t \cdot(R(t)-\mathbf{1}(t)) d t-\frac{1}{8}=\int_{\mathbb{R}} t \cdot(F(2 t-a)-F(2 t)) d t .
$$

It is easy to see that the right-hand side only vanishes when $a=0$, so we must have $\Phi=F$.

\subsection{Radially Symmetric Potentials}

We now prove Theorem 1.8. We start with a simple lemma.

Lemma 6.8 Assume that $Q$ is radially symmetric. Fix a point $p \in \partial S$, and rescale in the outwards normal direction (see (1.4)). Then every limiting kernel in Theorem 1.1 takes the form $K=G \Psi$, where $\Psi(z, w)=\Phi(z+\bar{w})$ is translation invariant.

Proof We can suppose that $p=1 \in \partial S$, and we rescale about $p=1$. Set $\delta=\Delta Q(1)$. By assumption, we have

$$
\mathbf{K}_{n}(\zeta, \eta)=\mathbf{K}_{n}(\kappa \zeta, \kappa \eta), \quad \text { where } \kappa=e^{i t / \sqrt{n \delta}}
$$

Now write $z=\sqrt{n \delta}(\zeta-1), w=\sqrt{n \delta}(\eta-1)$. Then

$$
\begin{aligned}
\kappa \zeta= & \left(1+i \frac{t}{\sqrt{n \delta}}+O\left(\frac{1}{n}\right)\right)\left(1+\frac{z}{\sqrt{n \delta}}\right)=1+\frac{1}{\sqrt{n \delta}}(z+i t) \\
& +O\left(\frac{1}{n}\right), \quad(n \rightarrow \infty) .
\end{aligned}
$$


This means that $\Psi_{n}(z, w)=\Psi_{n}(z+i t+o(1), w+i t+o(1))$, where $o(1) \rightarrow 0$ as $n \rightarrow \infty$.

Now assume that $Q$ is radially symmetric and that the droplet $S$ is connected; thus it is either a disc or an annulus. If $p \in \partial S$ is a boundary point, then the outer normal $N_{p}$ is simply a multiple of $p, N_{p}= \pm p /|p|$. We can assume that $|p|=1$ and $N_{p}=p$. Let us write

$$
R_{n, p}(t)=\frac{1}{n \Delta Q(p)} \mathbf{R}_{n}\left(p\left(1+\frac{t}{\sqrt{n \Delta Q(p)}}\right)\right)
$$

for the 1-point function rescaled about $p$. The radial symmetry of $Q$ implies that $R_{n, p}=R_{n, p e^{i \theta}}$ for all real $\theta$. From this we conclude that the almost-everywhere convergence in Theorem 1.5 must hold pointwise; i.e., if $R=\lim R_{n_{k}, p}$ is any limiting 1-point function, then

$$
\int_{\mathbb{R}} t \cdot(R(t)-\mathbf{1}(t)) d t=\frac{1}{8}
$$

In view of Lemma 6.8, we know that $R$ corresponds to a $t . i$. limiting kernel $K(z, w)=$ $G(z, w) \Phi(z+\bar{w})$. An application of Theorem 6.7 now shows that $\Phi=F$ is the plasma function. The proof of Theorem 1.8 is finished.

\subsection{Translation Invariant Solutions to the Mass-One Equation.}

We now prove Theorem 1.9.

Let $\Phi$ be an entire function of the form $\Phi=\gamma * f$, where $f$ is some bounded function.

Using Lemma 6.4 and the assumption that $\Psi(z, w)=\Phi(z+\bar{w})$, we can rewrite the mass-one equation (equality in (1.6)) in terms of the function $J=\left.\Phi\right|_{\mathbb{R}}$, as follows:

$$
\begin{aligned}
J(x) & =\int e^{-|w|^{2}}|\Psi(x / 2, x / 2+w)|^{2} d A(w)=\int e^{-|w|^{2}}|\Phi(x+w)|^{2} d A(w) \\
& =\frac{1}{(2 \pi)^{2}} \int_{\mathbb{C}} e^{-|w|^{2}} d A(w) \iint_{\mathbb{R}^{2}} e^{i(x+w) t} e^{i(x+\bar{w}) s} \hat{J}(t) \hat{J}(s) d s d t \\
& =\frac{1}{(2 \pi)^{2}} \iint_{\mathbb{R}^{2}} e^{i x(s+t)} e^{-s t} \hat{J}(t) \hat{J}(s) d s d t .
\end{aligned}
$$

(i) If $J=\gamma * \mathbf{1}_{E}$, where $E \subset \mathbb{R}$ is a Borel set of positive measure, then $\hat{J}(\xi)=$ $e^{-\xi^{2} / 2} \cdot \hat{\mathbf{1}}_{E}(\xi)$ in the sense of distributions. Passing to Fourier transforms, we find that the mass-one equation is equivalent to that (with $\delta$ the Dirac delta function)

$$
\begin{aligned}
e^{-\xi^{2} / 2} \hat{\mathbf{1}}_{E}(\xi) & =\frac{1}{2 \pi} \iint_{\mathbb{R}^{2}} e^{-s t} \delta(s+t-\xi) e^{-s^{2} / 2} \hat{\mathbf{1}}_{E}(s) e^{-t^{2} / 2} \hat{\mathbf{1}}_{E}(t) d s d t \\
& =\frac{1}{2 \pi} \int e^{-\xi^{2} / 2} \hat{\mathbf{1}}_{E}(s) \hat{\mathbf{1}}_{E}(\xi-s) d s=e^{-\xi^{2} / 2} \frac{1}{2 \pi}\left[\hat{\mathbf{1}}_{E} * \hat{\mathbf{1}}_{E}\right](\xi)
\end{aligned}
$$


By Fourier inversion, this is equivalent to $\mathbf{1}_{E}=\mathbf{1}_{E}^{2}$, which is true. We have shown that the function $J=\gamma * \mathbf{1}_{E}$ satisfies the mass-one equation.

(ii) If $\Psi(z, w)=\Phi(z+\bar{w})$ satisfies the mass-one equation and $\Phi=\gamma * f$, then the same calculations as above with " $\mathbf{1}_{E}$ " replaced by " $f$ " lead to the equation

$$
e^{-\xi^{2} / 2} \hat{f}(\xi)=e^{-\xi^{2} / 2} \frac{1}{2 \pi}[\hat{f} * \hat{f}](\xi) .
$$

Taking inverse Fourier transforms, we see that this is equivalent to that $f(x)=f(x)^{2}$ almost everywhere. Hence $f=\mathbf{1}_{E}$ almost everywhere, where $E$ is some measurable set of positive measure, and $\Phi=\mathbf{1}_{E} * \gamma$. The proof of Theorem 1.9 is finished.

\section{Ward's Equations in Some Other Settings}

In this section, we will rescale Ward identities and derive the corresponding equations in several different settings.

\subsection{Ward's Equation at the Hard Edge of the Spectrum}

For simplicity, we shall restrict our discussion to the hard edge Ginibre ensemble; we refer to [5] for a discussion of more general hard edge ensembles.

Let $\left\{\zeta_{j}\right\}_{1}^{n}$ be the hard-edge Ginibre process, and rescale about the boundary point $p=1$ to obtain the boundary process $\Theta_{n}=\left\{z_{j}\right\}_{1}^{n}$, where $z_{j}=\sqrt{n}\left(\zeta_{j}-1\right)$.

As before, we let $R_{n}(z)=K_{n}(z, z)$ denote the 1-point function of the rescaled process. The hard edge Berezin kernel and Cauchy transform are defined, respectively, by

$$
B_{n}(z, w)=\frac{\left|K_{n}(z, w)\right|^{2}}{K_{n}(z, z)}, \quad C_{n}(z)=\int_{\mathbb{C}} \frac{B_{n}(z, w)}{z-w} d A(w),
$$

with the understanding that $B_{n}(z, w)=0$ when the point $\zeta=1+z / \sqrt{n}$ satisfies $|\zeta|>1$.

We recall that the hard edge kernel is defined by (0.4). In terms of this kernel, we put

$$
R(z):=K(z, z), \quad B(z, w):=\frac{|K(z, w)|^{2}}{K(z, z)}, \quad C(z):=\int_{\mathbb{L}} \frac{B(z, w)}{z-w} d A(w)
$$

Observe that $R(z)=B(z, z)=0$ when $\operatorname{Re} z>0$.

Theorem 7.1 The hard edge kernel (0.4) satisfies Ward's equation

$$
\bar{\partial} C(z)=R(z)-1-\Delta \log R(z), \quad z \in \mathbb{L}:=\{z ; \operatorname{Re} z<0\} .
$$


Proof We claim first that we have the asymptotic relation

$$
\bar{\partial} C_{n}(z)=R_{n}(z)-1-\Delta \log R_{n}(z)+o(1), \quad z \in \mathbb{L},
$$

where the error term $o(1)$ converges to zero uniformly on compact subsets of $\mathbb{L}$.

In order to prove this, it is convenient to consider the Ginibre potential $Q(\zeta)=$ $|\zeta+1|^{2}$ which has the droplet $S=\{|\zeta+1| \leq 1\}$. We rescale about the boundary point $p=0$ via $z=\sqrt{n} \zeta$.

Fix a number $\varepsilon>0$. Write $U:=S \cap D(0 ; \varepsilon)$, and consider test functions $\psi$ supported in the dilated set $\sqrt{n} \cdot U$. As in the free case, we define $\psi_{n}(\zeta):=\psi(z)$. Since $Q^{S}=Q$ in the set $U$ where $\psi_{n}$ is supported, the same arguments used in the free boundary case remain valid (cf. Subsection 4.2). The only difference is that the dilated domains $\sqrt{n} \cdot U$ will, in our present case, increase to the open left half-plane $\mathbb{L}$. Hence we deduce Ward's equation (7.1) for $z \in \mathbb{L}$ precisely as before.

By Theorem 2.3, we have convergence $R_{n} \rightarrow R$ and $C_{n} \rightarrow C$ locally uniformly in $\mathbb{L}$ and boundedly almost everywhere in $\mathbb{C}$. It follows that we can pass to the limit in (7.1).

Corollary 7.2 The hard edge kernel (0.4) satisfies the mass-one equation

$$
\int_{\mathbb{L}} B(z, w) d A(w)=1, \quad(z \in \mathbb{L}) .
$$

Proof The approximate Berezin kernels $B_{n}$ satisfy $\int B_{n}(z, w) d A(w)=1$ for $z \in \mathbb{L}$. The identity (7.2) now follows from the convergence $B_{n} \rightarrow B$ in Theorem 2.3 and the argument used in the foregoing proof.

Remark A different hard-edge solution to Ward's equation in a strip is given in the last section of [6]. Cf. the forthcoming paper [5] for details.

\subsection{Ward's Equation at Bulk Singularities and Mittag-Leffler Fields}

Let us weaken our standing assumptions on the potential $Q$. We still require realanalyticity in a neighborhood of $S$, but now allow that $\Delta Q=0$ at isolated points in the bulk of $S$. A point $p \in$ Int $S$ such that $\Delta Q(p)=0$ will be called a bulk singularity.

Assume that $p=0$ is a bulk singularity, and let $\left\{\zeta_{j}\right\}_{1}^{n}$ be the point process corresponding to $Q$. The effect of the bulk singularity is to repel the particles away from it.

There are various types of bulk singularities depending on the local behavior of $\Delta Q$ near $p$. For instance, if $\Delta Q(\zeta)=a x^{2}+b y^{2}+O\left(|\zeta|^{3}\right)$ as $\zeta=x+i y \rightarrow 0$, where $a$ and $b$ are positive constants, then the local behavior of the system $\left\{\zeta_{j}\right\}$ near 0 will depend on $a$ as well as $b$. Let us consider the symmetric case when $a=b=1$, or more generally, that there is a number $\lambda \geq 1$ such that

$$
\Delta Q(\zeta)=|\zeta|^{2(\lambda-1)}+\ldots, \quad(\zeta \rightarrow 0)
$$


where the dots represent negligible terms. If we wish $Q$ to be real-analytic, we should of course assume that $\lambda$ be an integer. However, the condition of real-analyticity is important only in a neighborhood of the boundary, e.g., in connection with Sakai's theory. In the bulk, it suffices to assume $C^{2}$-smoothness. Thus we can in fact choose $\lambda$ as an arbitrary real constant $\geq 1$. Note that $\lambda=1$ is the well-known case of an ordinary "regular" bulk point, in which case we know that the usual Ginibre point field arises. We may thus assume that $\lambda>1$.

It turns out that the proper scaling in the case at hand is

$$
z_{j}=n^{1 /(2 \lambda)} \zeta_{j}
$$

We write $\Theta_{n}=\left\{z_{j}\right\}_{1}^{n}$ for the rescaled system, equipped with the law that is the image of the Boltzmann-Gibbs measure $\mathbb{P}_{n}$ under the map (7.3).

Example Consider the "power potential" $Q_{\lambda}(\zeta)=|\zeta|^{2 \lambda}$, where $\lambda>1$. If $\mathbf{K}_{n}$ denotes a correlation kernel of the process $\left\{\zeta_{j}\right\}_{1}^{n}$, then $\Theta_{n}$ has the correlation kernel

$$
K_{n}(z, w)=n^{-1 / \lambda} \mathbf{K}_{n}(\zeta, \eta), \quad \text { where } z=n^{1 /(2 \lambda)} \zeta, \quad w=n^{1 /(2 \lambda)} \eta
$$

A straightforward calculation shows that the polynomial $\zeta^{j}$ has norm

$$
\left\|\zeta^{j}\right\|_{n Q}^{2}=\int_{\mathbb{C}}|\zeta|^{2 j} e^{-n|\zeta|^{2 \lambda}} d A(\zeta)=\frac{1}{\lambda} n^{-\frac{j+1}{\lambda}} \Gamma\left(\frac{j+1}{\lambda}\right) .
$$

Inserting the result in formula (1.3) for a correlation kernel, we get

$$
\mathbf{K}_{n}(\zeta, \eta)=\lambda n^{1 / \lambda} \sum_{j=0}^{n-1} \frac{\left(n^{1 / \lambda} \zeta \bar{\eta}\right)^{j}}{\Gamma\left(\frac{j+1}{\lambda}\right)} e^{-n\left(|\zeta|^{2 \lambda}+|\eta|^{2 \lambda}\right) / 2}
$$

Rescaling according to $z=n^{1 /(2 \lambda)} \zeta, w=n^{1 /(2 \lambda)} \eta$, we obtain

$$
K_{n}(z, w)=n^{-1 / \lambda} \mathbf{K}_{n}(\zeta, \eta)=\lambda \sum_{j=0}^{n-1} \frac{(z \bar{w})^{j}}{\Gamma\left(\frac{j+1}{\lambda}\right)} e^{-\left(|z|^{2 \lambda}+|w|^{2 \lambda}\right) / 2}
$$

It is now evident that

$$
K_{n}(z, w) \rightarrow M_{\lambda}(z \bar{w}) e^{-\left(|z|^{2 \lambda}+|w|^{2 \lambda}\right) / 2}, \quad(n \rightarrow \infty),
$$

locally uniformly in $\mathbb{C}^{2}$, where $M_{\lambda}$ is the function

$$
M_{\lambda}(z)=\lambda \sum_{j=0}^{\infty} \frac{z^{j}}{\Gamma\left(\frac{j+1}{\lambda}\right)}
$$


We recognize $M_{\lambda}$ as the generalized Mittag-Leffler function $\lambda E_{1 / \lambda, 1 / \lambda}$. See [20]. (A similar computation was carried out in [40].)

In the next theorem, we consider Ward's equation at $p=0$ for the potential $Q_{\lambda}(\zeta)=$ $|\zeta|^{2 \lambda}$. To this end, we introduce the Berezin kernel rescaled about 0 on the scale (7.3), i.e., $B_{n}(z, w):=\left|K_{n}(z, w)\right|^{2} / K_{n}(z, z)$.

Theorem 7.3 The point process $\Theta_{n}$ converges as $n \rightarrow \infty$ to the unique point-field $M L_{\lambda}$ in $\mathbb{C}$ with kernel

$$
K(z, w)=M_{\lambda}(z \bar{w}) e^{-\frac{1}{2}\left(|z|^{2 \lambda}+|w|^{2 \lambda}\right)} .
$$

The convergence holds in the sense of locally uniform convergence of intensity functions. Furthermore, $B_{n} \rightarrow B$ uniformly on compact subsets of $\mathbb{C}^{2}$ where B is a solution to the Ward's equation

$$
\bar{\partial}_{z} \int \frac{B(z, w)}{z-w} d A(w)=B(z, z)-\lambda^{2}|z|^{2(\lambda-1)}-\Delta_{z} \log B(z, z) .
$$

Proof It is easy to see that $M_{\lambda}$ is of exponential type $\lambda$. This implies that the kernel $K$ is uniformly bounded. Existence and uniqueness of a point field $M L_{\lambda}$ with the given properties now follows, via Lenard's theory, from the convergence of intensities in the preceding example.

We shall establish the asymptotic relation

$$
\bar{\partial}_{z} \int \frac{B_{n}(z, w)}{z-w} d A(w)=B_{n}(z, z)-\lambda^{2}|z|^{2(\lambda-1)}-\Delta_{z} \log B_{n}(z, z)+o(1),
$$

where $o(1) \rightarrow 0$ as $n \rightarrow \infty$, uniformly on compact subsets of $\mathbb{C}$.

To this end, fix a test function $\psi$, and let $\psi_{n}(\zeta)=\psi(z)$, where $z=n^{1 /(2 \lambda)} \zeta$.

We shall use Ward's identity; we therefore recalculate the expectations of the terms $\mathrm{I}_{n}\left[\psi_{n}\right], \mathrm{II}_{n}\left[\psi_{n}\right]$, and $\mathrm{III}_{n}\left[\psi_{n}\right]$ used in the free boundary case, in Subsection 4.2. As customary, we use the symbol $\mathbf{R}_{n, k}$ to denote the $k$-point function of the system $\left\{\zeta_{j}\right\}_{1}^{n}$. The rescaling $z_{j}=n^{1 /(2 \lambda)} \zeta_{j}$ then implies that the $k$-point function of the rescaled system $\left\{z_{j}\right\}_{1}^{n}$ is

$$
R_{n, k}\left(z_{1}, \ldots, z_{k}\right)=n^{-k / \lambda} \mathbf{R}_{n, k}\left(\zeta_{1}, \ldots, \zeta_{k}\right)
$$

For $\mathrm{I}_{n}\left[\psi_{n}\right]$, the change of variables in (7.4) gives that

$$
\begin{aligned}
\mathbb{E}_{n} \mathrm{I}_{n}\left[\psi_{n}\right] & =n^{1 /(2 \lambda)} \frac{1}{2} \iint \frac{\psi(z)-\psi(w)}{z-w} R_{n, 2}(z, w) \\
& =n^{1 /(2 \lambda)} \int \psi(z) d A(z) \int \frac{R_{n, 2}(z, w)}{z-w} d A(w) .
\end{aligned}
$$


Turning to $\mathrm{II}_{n}\left[\psi_{n}\right]$, we first observe that $\partial Q_{\lambda}(\zeta)=\lambda \bar{\zeta} \cdot|\zeta|^{2(\lambda-1)}$. Using this, we see that

$$
n \partial Q_{\lambda}\left(\frac{z}{n^{1 /(2 \lambda)}}\right)=n^{1 /(2 \lambda)} \partial Q_{\lambda}(z)
$$

which gives

$$
\mathbb{E}_{n} \mathrm{II}_{n}\left[\psi_{n}\right]=n^{1 /(2 \lambda)} \int \lambda \bar{z}|z|^{2(\lambda-1)} \cdot \psi \cdot R_{n, 1}
$$

We also compute

$$
\mathbb{E}_{n} \mathrm{III}_{n}\left[\psi_{n}\right]=n^{1 /(2 \lambda)} \int \partial \psi \cdot R_{n, 1}=-n^{1 /(2 \lambda)} \int \psi \cdot \partial R_{n, 1} .
$$

In view of Ward's identity (Subsection 4.1), we now infer that, in the sense of distributions,

$$
\int \frac{R_{n, 2}(z, w)}{z-w} d A(w)=\lambda \bar{z}|z|^{2(\lambda-1)} \cdot R_{n, 1}(z)+\partial R_{n, 1}(z) .
$$

Dividing by $R_{n, 1}$ and applying $\bar{\partial}$, we conclude the proof of the formula (7.5). To pass to the limit as $n \rightarrow \infty$, we now use the convergence in the example preceding Theorem 7.3 and the argument in Subsection 4.3.

More generally, it is natural to consider any bulk singularity where the equilibrium density vanishes as $\Delta Q(\zeta) \sim|\zeta|^{2 k-2}$ as $\zeta \rightarrow 0$, where $k$ is a positive integer. Rescaling by a suitable factor proportional to $n^{-1 / 2 k}$, one deduces the asymptotic relation

$$
\bar{\partial}_{z} \int \frac{B_{n}(z, w)}{z-w} d A(w)=B_{n}(z, z)-\Delta Q(z)-\Delta_{z} \log B_{n}(z, z)+o(1) .
$$

This equation is studied and applied in the paper [8].

An equally interesting generalization is obtained by allowing the potential to have a weak logarithmic singularity at the origin. This corresponds to the microscopic study of a particle system on a Riemann surface, close to a conical singularity, or alternatively, to the study of the microscopic effect of insertion of a point charge. This possibility is considered in the papers $[4,8]$ and will also be the subject of a forthcoming investigation.

\subsection{Ward's Equation and the Mass-One Equation for $\beta$-Ensembles}

Consider a potential $Q$ satisfying the standing assumptions in Subsection 1.1, and fix a number $\beta>0$. Let us consider the probability measure on $\mathbb{C}^{n}$ defined by 


$$
d \mathbb{P}_{n}^{\beta}(\zeta):=\frac{1}{Z_{n}^{\beta}} e^{-\beta H_{n}(\zeta)}, \quad Z_{n}^{\beta}:=\int_{\mathbb{C}^{n}} e^{-\beta H_{n}(\zeta)} d V_{n}(\zeta),
$$

where $H_{n}$ is the usual Hamiltonian, see (1.2).

Let $\mathbf{R}_{n, k}^{\beta}$ denote the corresponding $k$-point function. The following version of Ward's identity is proved exactly as in the case $\beta=1$.

Theorem 7.4 Put, for a test function $\psi \in C_{0}^{\infty}(\mathbb{C})$,

$$
W_{n}[\psi]=\beta\left(\mathrm{I}_{n}[\psi]-\mathrm{II}_{n}[\psi]\right)+\mathrm{III}_{n}[\psi],
$$

where $\mathrm{I}_{n}, \mathrm{II}_{n}, \mathrm{III}_{n}$ are as in Subsection 4.1. If $Q$ is $C^{2}$-smooth near supp $\psi$, then $\mathbb{E}_{n}^{\beta} W_{n}^{+}[\psi]=0$.

Now fix a point $p \in S$, and rescale about $p: z_{j}=e^{-i \theta} \sqrt{n \Delta Q(p)}\left(\zeta_{j}-p\right)$. We denote by $\Theta_{n}^{\beta}:=\left\{z_{j}\right\}_{1}^{n}$ the rescaled process and write $R_{n, k}^{\beta}\left(z_{1}, \ldots, z_{k}\right):=$ $\mathbf{R}_{n, k}^{\beta}\left(\zeta_{1}, \ldots, \zeta_{k}\right)$ for the joint intensities. We also define the Berezin kernel of the process $\Theta_{n}^{\beta}$ by

$$
B_{n}^{\beta}(z, w):=\frac{R_{n, 1}^{\beta}(z) R_{n, 1}^{\beta}(w)-R_{n, 2}^{\beta}(z, w)}{R_{n, 1}^{\beta}(z)} .
$$

Notice that $B_{n}^{\beta}(z, z)=R_{n, 1}^{\beta}(z)$ and $\int_{\mathbb{C}} B_{n}^{\beta}(z, w) d A(w)=1$.

Rescaling the Ward identity as in Subsection 4.2, we obtain the following result.

Theorem 7.5 If $p$ belongs to some neighborhood of $S$ in which $Q$ is strictly subharmonic and $C^{2}$-smooth, then

$$
\bar{\partial} C_{n}^{\beta}(z)=R_{n}^{\beta}(z)-1-\frac{1}{\beta} \Delta_{z} \log R_{n}^{\beta}(z)+o(1),
$$

where

$$
C_{n}^{\beta}(z):=\int_{\mathbb{C}} \frac{B_{n}^{\beta}(z, w)}{z-w} d A(w), \quad R_{n}^{\beta}:=R_{n, 1}^{\beta} .
$$

We do not know whether it is possible to pass to the limit as $n \rightarrow \infty$ in (7.6), but for the sake of argument, let us temporarily assume that we can define a limiting Berezin kernel $B^{\beta}$. Letting $n \rightarrow \infty$ in (7.6), one then formally obtains the following generalization of Ward's equation:

$$
\bar{\partial}_{z} \int_{\mathbb{C}} \frac{B^{\beta}(z, w)}{z-w} d A(w)=B^{\beta}(z, z)-1-\frac{1}{\beta} \Delta_{z} \log B^{\beta}(z, z), \quad z \in \mathbb{C} .
$$

This more general equation can easily be transformed to the case $\beta=1$ by the linear scaling

$$
B(u, v)=B^{\beta}(z, w), \quad u=\sqrt{\beta} z, v=\sqrt{\beta} w .
$$


Proposition 7.6 Suppose that $B^{\beta}$ solves (7.7). Then the kernel $B$ in (7.8) solves Ward's equation (with $\beta=1$ ).

We do not know whether the (presumptive) kernels $B^{\beta}$ would be non-negative, so speaking about "mass-one" could possibly be misleading. However, if we assume that $\int_{\mathbb{C}} B^{\beta}(z, w) d A(w)=1$, then the corresponding kernel $B$ in (7.8) satisfies the "mass- $\beta$ equation": $\int_{\mathbb{C}} B(u, v) d A(v)=\beta$.

As we mentioned before, in the case $\beta=1$, Ward's equation is "closed" by analytic continuation. We don't know if we can consider equation (7.8) closed if $\beta \neq 1$.

The study of boundary profiles $R^{\beta}(x)$ for a given $\beta>1$ is of physical relevance, in connection to the Hall effect; see the paper [13].

\section{Concluding Remarks}

In Subsection 8.1, we explain how the boundary kernel $K=G F$ in the Ginibre case can be related to asymptotics of section of the exponential function. In Subsection 8.2, we will mention some connections to the theory of Hilbert spaces of entire functions and to the theories of certain special functions. In Subsection 8.3, we comment on the nature of the mass-one equation and Ward's equation in the general (non-translationinvariant) case, relating those equations to harmonic analysis on the Heisenberg group.

\subsection{Sections of Power Series}

It seems that the type of asymptotics one encounters for the free boundary was first observed in connection with sections of power series of the exponential function. By a section of an entire function $f(\zeta)=\sum_{j=0}^{\infty} a_{j} \zeta^{j}$, we here simply mean a partial sum

$$
s_{n}(\zeta)=\sum_{j=0}^{n-1} a_{j} \zeta^{j}
$$

Szegó's original study in [36] concerns the distribution of zeros of the blow-up sections $s_{n}^{\sharp}(w):=s_{n}(n w)$ pertaining to the exponential function $f(\zeta)=e^{\zeta}$. In the course of the investigation, Szegó proves asymptotic results for the function $s_{n}^{\sharp}(w)$ valid for all $w$ except for $w$ in a fixed neighborhood of 1. This gap was later closed, and the following result ensued. Consider the rescaled section

$$
\tilde{s}_{n}(z):=s_{n}(n \zeta), \quad z=\sqrt{n}(\zeta-1)
$$

One then has the following (locally uniform) convergence

$$
\tilde{s}_{n}(z) e^{-n-\sqrt{n} z} \rightarrow F(z) \quad(n \rightarrow \infty)
$$

where $F$ is the plasma function (0.3). We are unsure concerning whom should be credited for the convergence in (8.1) when $f(\zeta)=e^{\zeta}$. However, the book [15, Theorem 1] 
contains a statement valid for more general $f$, and the appendix in [10] contains a detailed convergence result for the case at hand.

To see the connection with the scaling limits of the present paper, we remind the reader of the expression for the correlation kernel for the Ginibre ensemble from Section 2,

$$
\mathbf{K}_{n}(\zeta, \eta)=n s_{n}(n \zeta \bar{\eta}) e^{-n \frac{|\zeta|^{2}+|\eta|^{2}}{2}}
$$

Rescaling via $z=\sqrt{n}(\zeta-1), w=\sqrt{n}(\eta-1), K_{n}(z, w)=n^{-1} \mathbf{K}_{n}(\zeta, \eta)$, we now recognize that

$$
K_{n}(z, 0)=\tilde{s}_{n}(z) e^{-n} e^{-\sqrt{n} \operatorname{Re} z} \cdot e^{-|z|^{2} / 2} .
$$

Letting $c_{n}$ be the cocycle $c_{n}(z)=e^{-i \sqrt{n} \operatorname{Im} z}$, we now form the functions

$$
\tilde{K}_{n}(z, 0)=c_{n}(z) K_{n}(z, 0)=\tilde{s}_{n}(z) e^{-n-\sqrt{n} z} \cdot e^{-|z|^{2} / 2},
$$

which now closely resembles the left-hand side in (8.1). By Theorem 2.1 or Theorem 1.8 , we have

$$
\tilde{K}_{n}(z, w) \rightarrow G(z, w) F(z+\bar{w}) .
$$

Letting $w=0$ (and thus $\eta=1$ ), one can now recover the limit in (8.1).

The convergence in (8.1) has been proved for the sections corresponding to more general entire functions. In the monograph [15], the authors consider the MittagLeffler function $E_{1 / \lambda}$ as well as a class denoted " $\mathcal{L}$-functions." More recently, this kind of convergence has been used in the papers [29,39] (it is called "Newman-Rivlin asymptotics" in [39]).

To interpret the above results in terms of our Theorem 1.8, one chooses a suitable radially symmetric potential $Q$. For example, one chooses $Q(\zeta)=E_{1 / \lambda}\left(|\zeta|^{2}\right.$ ) in case of the Mittag-Leffler function alluded to above. Expressing the kernel $\mathbf{K}_{n}$ in terms of the orthogonal polynomials (as in (1.3)) and rescaling about a boundary point of the droplet, one can apply Theorem 1.8 and recover the asymptotic behavior of the sections.

\subsection{The Mass-One Equation and Hilbert Spaces of Entire Functions}

It has been observed (e.g., [11,26,27,37,38] and the references there) that universality laws in the theory of random Hermitian matrices are related to certain specific de Branges spaces $\mathcal{B}(E)$ of entire functions. See [14] for the definition of these spaces. In particular, the sine-kernel describing the spacing of eigenvalues in the bulk is the restriction to $\mathbb{R}^{2} \subset \mathbb{C}^{2}$ of the reproducing kernel of the Paley-Wiener space, i.e., the space $\mathcal{B}(E)$ where $E(z)=e^{-i \pi z}$. Moreover, the Airy kernel 


$$
K(x, y)=\frac{\operatorname{Ai}(x) \mathrm{Ai}^{\prime}(y)-\mathrm{Ai}^{\prime}(x) \operatorname{Ai}(y)}{x-y},
$$

which describes the spacing at the edge of the spectrum, is the restriction to $\mathbb{R}^{2}$ of the reproducing kernel of $\mathcal{B}(E)$ where $E=\mathrm{Ai}^{\prime}-i \mathrm{Ai}$, and the Bessel kernel (hard edge) is the restriction to $(-\infty, 0) \times(-\infty, 0)$ of the reproducing kernel of the de Branges space corresponding to the function $E(z)=\sqrt{z} J_{0}^{\prime}(\sqrt{z})-i J_{0}(\sqrt{z})$.

The appearance of de Branges spaces in the context of Hermitian random matrices is quite natural given the fact that orthogonal polynomials on the real line can be related to a second order one-dimensional self-adjoint spectral problem.

The Hilbert spaces $\mathcal{H}$ of entire functions arising in the random normal matrix theory are not of de Branges type, and we are not sure about their spectral interpretation. Nevertheless, we will use the term "spectral measure": $\mu$ is a spectral measure for $\mathcal{H}$ if $\mathcal{H}$ sits isometrically in $L^{2}(\mu)$.

Lemma 8.1 Let $\Psi$ be a Hermitian entire function and $G=G(z, w)$ the Ginibre kernel. The following conditions are equivalent:

(i) The kernel $K=G \Psi$ satisfies the mass-one equation; i.e.,

$$
\int e^{-|w|^{2}}|\Psi(z, z+w)|^{2} d A(w)=\Psi(z, z), \quad z \in \mathbb{C}
$$

(ii) The holomorphic kernel $L(z, w)=e^{z \bar{w}} \Psi(z, w)$ is the reproducing kernel of some Hilbert space $\mathcal{H}$ with spectral measure $d \mu(z):=e^{-|z|^{2}} d A(z)$.

If this is the case, then there is a unique point field with correlation kernel $K$.

Proof Write $L_{w}(z)=L(z, w)=e^{z \bar{w}} \Psi(z, w)$ (cf. Subsection 4.3). Then $L$ is the reproducing kernel for a Hilbert space with spectral measure $d \mu(z)=e^{-|z|^{2}} d A(z)$ if and only if

$$
\left\langle L_{w}, L_{z}\right\rangle_{L^{2}(\mu)}=L(z, w), \quad z, w \in \mathbb{C} .
$$

For $z=w$, the identity (8.3) means that

$$
\begin{aligned}
& \int_{\mathbb{C}}\left|L_{z}(\zeta)\right|^{2} e^{-|\zeta|^{2}} d A(\zeta)=e^{|z|^{2} \Psi(z, z)} \\
& \text { i.e., } \quad \int_{\mathbb{C}}|\Psi(\zeta, z)|^{2} e^{\zeta \bar{z}+\bar{\zeta} z-|\zeta|^{2}-|z|^{2}} d A(\zeta)=\Psi(z, z),
\end{aligned}
$$

which is precisely the mass-one equation (8.2). On the other hand, if the last equation holds, then (8.3) follows for $z \neq w$ by analytic continuation. This proves the equivalence of (i) and (ii).

Next note that the kernel $K=G \Psi$ can be written

$$
K(z, w)=e^{-|z|^{2} / 2-|w|^{2} / 2} L(z, w) .
$$


From this we conclude that if $L$ gives rise to a reproducing kernel as in (ii), then $K$ is the reproducing kernel of the subspace $\mathcal{W}=\left\{f ; f(z)=g(z) e^{-|z|^{2} / 2}, g \in \mathcal{H}\right\}$ of $L^{2}$.

Consider the linear operator $T$ on $L^{2}$ with kernel $K$. Then $T$ is an orthogonal projection, and it is locally trace class. (That an operator $T$ on $L^{2}$ is "locally trace class" means that the operator $T_{B}$ on $L^{2}$ defined by $T_{B}(f)=T\left(\mathbf{1}_{B} f\right)$ is trace class for every compact set $B \subset \mathbb{C}$.) By a theorem of Soshnikov ( [34, Theorem 3]), the conditions above guarantee that $K$ is the correlation kernel of a unique random point field in $\mathbb{C}$.

In the following, we write $A^{2}(\mu)$ for the space of all entire functions of class $L^{2}(\mu)$. It follows from general facts for reproducing kernels that the Hilbert space $\mathcal{H}$ in (ii) is the closed linear span

$$
\mathcal{H}=\operatorname{span}_{L^{2}(\mu)}\left\{L_{w} ; w \in \mathbb{C}\right\}, \quad\left(d \mu(z)=e^{-|z|^{2}} d A(z)\right)
$$

Let us look at some examples.

For the bulk Ginibre process, we have $L(z, w)=e^{z \bar{w}}$, and hence $\mathcal{H}=L_{a}^{2}(\mu)$ is the Fock space. The free boundary Ginibre process corresponds to the kernel $L(z, w)=$ $e^{z \bar{w}} F(z+\bar{w})$, and hence

$$
\mathcal{H}=\operatorname{span}_{L^{2}(\mu)}\left\{e^{\bar{w} z} F(z+\bar{w}) ; w \in \mathbb{C}\right\},
$$

where $F$ is the plasma function (0.3).

One can similarly interpret the hard edge mass-one equation (7.2) as a reproducing property in a suitable space of entire functions. In fact, this space is

$$
\mathcal{H}=\operatorname{span}_{L^{2}\left(\mu_{h}\right)}\left\{e^{\bar{w} z} H(z+\bar{w}) ; w \in \mathbb{L}\right\}, \quad\left(d \mu_{h}(z)=e^{-|z|^{2}} \cdot \mathbf{1}_{\mathbb{L}}(z) d A(z)\right),
$$

where $H$ is the hard edge function (0.5). The fact that the last span consists of entire functions requires a compactness property in the hard edge situation, which will be established in the paper [5].

It would be interesting to describe the above spaces in more constructive terms (e.g., similar to de Branges theory). It would also be interesting to know the meaning of Ward's equation for the spaces $\mathcal{H}$. (By Lemma 8.1, the mass-one equation is a statement about spectral measures.)

We finally describe the Hilbert spaces corresponding to the Mittag-Leffler processes. To this end, recall that the mass-one equation for the function $M_{\lambda}$ says that

$$
\int_{\mathbb{C}}\left|M_{\lambda}(z \bar{w})\right|^{2} e^{-|w|^{2 \lambda}} d A(w)=M_{\lambda}\left(|z|^{2}\right) .
$$

This gives, by polarization

$$
\int_{\mathbb{C}} M_{\lambda}\left(z_{1} \bar{w}\right) M_{\lambda}\left(\bar{z}_{2} w\right) e^{-|w|^{2 \lambda}} d A(w)=M_{\lambda}\left(z_{1} \bar{z}_{2}\right) .
$$


(This formula has an alternative, elementary proof: insert $M_{\lambda}(\zeta)=\lambda \sum \zeta^{j} / \Gamma\left(\frac{j+1}{\lambda}\right)$ in the left-hand side and integrate termwise.) thus

Let $d \mu_{\lambda}(z)=e^{-|z|^{2 \lambda}} d A(z)$. The Hilbert space pertaining to the process $M L_{\lambda}$ is

$$
\mathcal{H}:=\operatorname{span}_{L^{2}\left(\mu_{\lambda}\right)}\left\{z \mapsto M_{\lambda}(z \bar{w}) ; w \in \mathbb{C}\right\}
$$

It is not hard to show that polynomials are dense in $\mathcal{H}$, and consequently $\mathcal{H}=A^{2}\left(\mu_{\lambda}\right)$.

In general, we expect that a kernel $L$ arising from rescaling in a (free-boundary) ensemble should be the Bergman kernel of a subspace of a "generalized Fock-Sobolev space"; see $[7,8]$ for results in this direction.

\subsection{Twisted Convolutions}

We finally show that, without the hypothesis of translation invariance, Ward's equation takes the form of a so-called twisted convolution equation, known from Weyl's calculus for pseudodifferential operators. A solution of this equation, coupled with the a priori estimates above, could plausibly lead to a proof of the hypothesis of translation invariance (say, at a regular boundary point). This thread will be taken up elsewhere.

For two functions $f, g$ defined on $\mathbb{C}$, we define the twisted convolution $f \star g$ by

$$
(f \star g)(z):=\int_{\mathbb{C}} f(z-w) g(w) e^{i \operatorname{Im}(\bar{z} w)} d A(w) .
$$

See the book [16]. We will show that Ward's equation and the mass-one equation can be interpreted as twisted convolution equations. In the translation invariant case, the equations reduce to usual convolution equations, which is how we were able to solve them. However, the general twisted case is certainly more interesting.

Consider the following transform:

$$
\hat{f}(t):=\int_{\mathbb{C}} e^{-i(z, t)} f(z) d A(z), \quad t \in \mathbb{C}, \quad(z, t):=2 \operatorname{Re}(z \bar{t}) .
$$

Letting $\mathcal{F}$ be the two-dimensional Fourier transform with normalization

$$
\mathcal{F}[f](u+i v)=\frac{1}{2 \pi} \int_{\mathbb{C}} e^{-i(x u+y v)} f(x+i y) d x d y,
$$

we then have $\hat{f}(t)=2 \mathcal{F}[f](2 t)$, and the inverse Fourier transform takes the form

$$
f(z)=\int_{\mathbb{C}} e^{i(z, t)} \hat{f}(t) d A(t) .
$$

Let $K=G \Psi$ denote a limiting kernel in Theorem 1.1, and write $R(z)=\Psi(z, z)$. We expect that, with a suitable interpretation of the transform, there is a function $f$ such that 


$$
\hat{R}=\hat{f} \cdot \Gamma, \quad \text { where } \Gamma(z):=e^{-|z|^{2} / 2} / \sqrt{2 \pi} .
$$

Here $\hat{f}$ is understood in the sense of tempered distributions.

By polarizing in the Fourier inversion formula, we obtain an analogue of the identity (6.4):

Lemma 8.2 For all $z, w \in \mathbb{C}$, we have

$$
\Psi(z, w)=\int_{\mathbb{C}} e^{i(z \bar{t}+\bar{w} t)} \hat{R}(t) d A(t) .
$$

Now define $R_{0}=1-R$, and assume that we can represent $R_{0}$ in a similar way to (8.4) $\hat{R}_{0}=\hat{g} \cdot \Gamma$, where $g$ is a suitable function. Lemma 8.2 then allows us to rewrite the mass-one equation and Ward's equation as follows.

Mass-one equation.

$$
\iint_{\mathbb{C}^{2}} e^{-\bar{s} t} e^{i(z, s+t)} \hat{R}(s) \hat{R}_{0}(t) d A(s) d A(t)=0, \quad(z \in \mathbb{C}) .
$$

Compare with Subsection 6.5 for the translation invariant analogue.

Ward's equation. There exists a smooth function $P_{0}$ such that $\bar{\partial} P_{0}=R_{0}$ and

$$
\iint_{\mathbb{C}^{2}} e^{-\bar{s} t} e^{i(z, s+t)} \hat{R}(s) \hat{P}_{0}(t) d A(s) d A(t)=0, \quad(z \in \mathbb{C}) .
$$

\section{Compare with Lemma 6.6.}

Note that both equations take the form

$$
\iint_{\mathbb{C}^{2}} e^{-\bar{s} t} e^{i(z, s+t)} \hat{F}(s) \hat{G}(t) d A(s) d A(t)=0 .
$$

If we here represent $\hat{F}=\hat{f} \cdot \Gamma, \hat{G}=\hat{g} \cdot \Gamma$, then (8.5) has the form of the twisted convolution equation $\hat{f} \star \hat{g}=0$.

Acknowledgements We thank Alexei Borodin and Misha Sodin for their interest. We also thank Aron Wennman, Seong-Mi Seo, Hee-Joon Tak, and Sungsoo Byun for careful reading and much appreciated help with improving this manuscript.

Open Access This article is distributed under the terms of the Creative Commons Attribution 4.0 International License (http://creativecommons.org/licenses/by/4.0/), which permits unrestricted use, distribution, and reproduction in any medium, provided you give appropriate credit to the original author(s) and the source, provide a link to the Creative Commons license, and indicate if changes were made.

\section{References}

1. Ameur, Y.: A density theorem for weighted Fekete sets. Int. Math. Res. Not. 16, 5010-5046 (2017)

2. Ameur, Y., Hedenmalm, H., Makarov, N.: Fluctuations of eigenvalues of random normal matrices. Duke Math. J. 159, 31-81 (2011)

3. Ameur, Y., Hedenmalm, H., Makarov, N.: Random normal matrices and Ward identities. Ann. Probab. 43(3), 1157-1201 (2015) 
4. Ameur, Y., Kang, N.-G.: On a problem for Ward's equation with a Mittag-Leffler potential. Bull. Sci. Math. 137(7), 968-975 (2013)

5. Ameur, Y., Kang, N.-G., Makarov, N.: Random normal matrix ensembles with a hard edge of the spectrum (to appear)

6. Ameur, Y., Kang, N.-G., Makarov, N., Wennman, A.: Scaling limits of random normal matrix processes at singular boundary points. arXiv: 1510.08723

7. Ameur, Y., Seo, S.-M.: Microscopic densities and Fock-Sobolev spaces. J. Anal. Math. (to appear) arXiv: 1610.10052

8. Ameur, Y., Seo, S.-M.: On bulk singularities in the random normal matrix model. Constr. Approx. 47, 3-37 (2018)

9. Aronszajn, N.: Theory of reproducing kernels. Trans. Am. Math. Soc. 68, 337-404 (1950)

10. Bleher, P., Mallison Jr., R.: Zeros of sections of exponential sums. Int. Math. Res. Not., Art. ID 38937 , 49 (2006)

11. Blower, G.: Operators associated with soft and hard spectral edges from unitary ensembles. J. Math. Anal. Appl. 337(1), 239-265 (2008)

12. Borodin, A., Sinclair, C.D.: The Ginibre ensemble of real random matrices and its scaling limits. Commun. Math. Phys. 291(1), 177-224 (2009)

13. Can, T., Forrester, P.J., Téllez, G., Wiegmann, P.: Singular behavior at the edge of Laughlin states. Phys. Rev. B 89, 235137 (2014)

14. de Branges, L.: Hilbert Spaces of Entire Functions. Prentice-Hall Inc, Englewood Cliffs (1968)

15. Edrei, A., Saff, E.B., Varga, R.S.: Zeros of Sections of Power Series, Volume 1002 of Lecture Notes in Mathematics. Springer, Berlin (1983)

16. Folland, G.B.: Harmonic Analysis in Phase Space, Volume 122 of Annals of Mathematics Studies. Princeton University Press, Princeton (1989)

17. Forrester, P.J.: Log-Gases and Random Matrices, Volume 34 of London Mathematical Society Monographs Series. Princeton University Press, Princeton (2010)

18. Forrester, P.J., Honner, G.: Exact statistical properties of the zeros of complex random polynomials. J. Phys. A 41, 375003 (1999)

19. Fried, B.D., Conte, S.D.: The Plasma Dispersion Function. The Hilbert Transform of the Gaussian. Academic Press, New York (1961)

20. Gorenflo, R., Kilbas, A.A., Mainardi, F., Rogosin, S.V.: Mittag-Leffler Functions, Related Topics and Applications. Springer Monographs in Mathematics. Springer, Heidelberg (2014)

21. Hedenmalm, H., Wennman, A.: Planar orthogonal polynomials and boundary universality in the random normal matrix model. arxiv: 1710.06493

22. Hörmander, L.: Notions of Convexity, Volume 127 of Progress in Mathematics. Birkhäuser, Boston (1994)

23. Hough, J.B., Krishnapur, M., Peres, Y., Virág, B.: Zeros of Gaussian Analytic Functions and Determinantal Point Processes, Volume 51 of University Lecture Series. American Mathematical Society, Providence (2009)

24. Katznelson, Y.: An Introduction to Harmonic Analysis. Cambridge Mathematical Library, 3rd edn. Cambridge University Press, Cambridge (2004)

25. Lee, S.-Y., Riser, R.: Fine asymptotic behavior for eigenvalues of random normal matrices: ellipse case. J. Math. Phys. 57(2), 023302, 29 (2016)

26. Lubinsky, D.S.: Universality limits for random matrices and de Branges spaces of entire functions. J. Funct. Anal. 256(11), 3688-3729 (2009)

27. Lubinsky, D.S.: An operator associated with de Branges spaces and universality limits. In: Agranovsky, M., Ben-Artzi, M., Galloway, G., Karp, L., Riech, S., Shoikhet, D., Weinstein, G., Zalcman, L. (eds.) Complex Analysis and Dynamical Systems IV. Part 1, Volume 553 of Contemporary Mathematics, pp. 213-229. American Mathematical Society, Providence (2011)

28. Mehta, M.L.: Random Matrices, Volume 142 of Pure and Applied Mathematics (Amsterdam), 3rd edn. Elsevier, Amsterdam (2004)

29. Norfolk, T.S.: Some observations on the Saff-Varga width conjecture. Rocky Mt. J. Math. 21, 529-538 (1991)

30. Rider, B.: A limit theorem at the edge of a non-Hermitian random matrix ensemble. J. Phys. A 36(12), 3401-3409 (2003). (Random matrix theory)

31. Riser, R.: Universality in gaussian random normal matrices. arXiv: 1302.0068 
32. Saff, E.B., Totik, V.: Logarithmic Potentials with External Fields, Volume 316 of Grundlehren der Mathematischen Wissenschaften. Springer, Berlin (1997). (Appendix B by Thomas Bloom)

33. Sakai, M.: Regularity of a boundary having a Schwarz function. Acta Math. 166(3-4), 263-297 (1991)

34. Soshnikov, A.: Determinantal random point fields. Uspekhi Mat. Nauk. 55(5(335)), 107-160 (2000)

35. Stein, E.M., Shakarchi, R.: Real Analysis, Volume 3 of Princeton Lectures in Analysis. Princeton University Press, Princeton (2005)

36. Szegő, G.: Über eine Eigenschaft der Exponentialreihe. Sitzungber. Berlin Math. Gessellschaftwiss 23, 50-64 (1924)

37. Tracy, C.A., Widom, H.: Level-spacing distributions and the Airy kernel. Commun. Math. Phys. 159(1), 151-174 (1994)

38. Tracy, C.A., Widom, H.: Level spacing distributions and the Bessel kernel. Commun. Math. Phys. 161(2), 289-309 (1994)

39. Vargas, A.R.: Newman-Rivlin asymptotics for partial sums of power series. arXiv:1503.0426v1

40. Veneziani, A.M., Pereira, T., Marchetti, D.H.U.: Conformal deformation of equilibrium measures in normal random ensembles. J. Phys. A 44, 075202 (2011) 